\title{
Sub-Chandrasekhar Mass Models For Supernovae
}

\author{
S. E. Woosley ${ }^{1}$ and Daniel Kasen ${ }^{2,3}$
}

\begin{abstract}
For carbon-oxygen white dwarfs accreting hydrogen or helium at rates in the range $\sim 1-10 \times 10^{-8} \mathrm{M}_{\odot} \mathrm{y}^{-1}$, a variety of explosive outcomes is possible well before the star reaches the Chandrasekhar mass. These outcomes are surveyed for a range of white dwarf masses $\left(0.7-1.1 \mathrm{M}_{\odot}\right)$, accretion rates $\left(1-7 \times 10^{-8}\right.$ $\left.\mathrm{M}_{\odot} \mathrm{y}^{-1}\right)$, and initial white dwarf temperatures $\left(0.01\right.$ and $\left.1 \mathrm{~L}_{\odot}\right)$. The results are particularly sensitive to the convection that goes on during the last few minutes before the explosion. Unless this convection maintains a shallow temperature gradient, and unless the density is sufficiently high, the accreted helium does not detonate. Below a critical helium ignition density, which we estimate to be $5-10 \times 10^{5} \mathrm{~g} \mathrm{~cm}^{-3}$, either helium novae or helium deflagrations result. The hydrodynamics, nucleosynthesis, light curves, and spectra of a representative sample of detonating and deflagrating models are explored. Some can be quite faint indeed, powered at peak for a few days by the decay of ${ }^{48} \mathrm{Cr}$ and ${ }^{48} \mathrm{~V}$. Only the hottest, most massive white dwarfs considered with the smallest helium layers, show reasonable agreement with the light curves and spectra of common Type Ia supernovae. For the other models, especially those involving lighter white dwarfs, the helium shell mass exceeds $0.05 \mathrm{M}_{\odot}$ and the mass of the ${ }^{56} \mathrm{Ni}$ that is synthesized exceeds $0.01 \mathrm{M}_{\odot}$. These explosions do not look like ordinary Type Ia supernovae, or any other frequently observed transient.
\end{abstract}

Subject headings: supernovae: general; supernovae: nucleosynthesis

\section{INTRODUCTION}

The possibility that explosive burning initiated in the thick helium shell of an accreting carbon-oxygen white dwarf (CO-dwarf) might lead to a supernova-like transient has been

\footnotetext{
${ }^{1}$ Department of Astronomy and Astrophysics, University of California, Santa Cruz, CA 95064; woosley@ucolick.org

${ }^{2}$ Departments of Physics and Astronomy, University of California, Berkeley, CA 94720

${ }^{3}$ Nuclear Science Division, Lawrence Berkeley National Laboratory, Berkeley, CA
} 
explored extensively in the literature. In the early 80's, it was realized that, for a range of accretion rates around $10^{-8} \mathrm{M}_{\odot} \mathrm{y}^{-1}$, a thick helium layer would accumulate and ignite explosively (Taam 1980a, b; Nomoto 1980, 1982a, b; Woosley et al. 1980). In some cases, the helium runaway ignited as a single detonation producing a faint supernova and leaving behind an intact white dwarf. In others, the helium detonation led to a secondary explosion of the CO core as well, and the star was completely disrupted (Nomoto 1980; Woosley et al. 1980; Nomoto 1982b; Livne 1990). Detailed studies of the energetics, nucleosynthesis and light curves of these explosions were carried out (e.g., Woosley et al. 1986; Woosley \& Weaver 1994). A critical density for the detonation of helium in this context was noted, $1-2 \times 10^{6} \mathrm{~g}$ $\mathrm{cm}^{-3}$ (Nomoto 1982b), as was the possibility of intrinsically faint Type Ia supernovae. The key role of such supernovae in synthesizing ${ }^{44} \mathrm{Ca}$ (as its radioactive progenitor ${ }^{44} \mathrm{Ti}$ ) was also discovered (Woosley \& Weaver 1994).

Exploratory multi-dimensional studies of this model were carried out in the 90's (Livne \& Glasner 1990, 1991; Livne \& Arnett 1995; Arnett 1997; Benz 1997; Livne 1997; García-Senz et al. 1999), in both two and three dimensions. A critical issue was whether helium detonation, if it occurred, would lead to carbon detonation and the explosion of the whole star. The general consensus was that it would, but there were a variety of caveats. First, it was much easier to ignite a carbon detonation if the helium detonation did not occur right at the COhelium interface, but at some altitude above it. A mixed layer between $\mathrm{CO}$ and helium also favored detonation. The helium layers considered were also all rather thick, at least $0.1 \mathrm{M}_{\odot}$. If "direct drive" did not work, a carbon detonation might still occur by the focusing, within the CO-core, of compressional waves caused by helium detonation, providing that the zoning in the calculation was fine enough.

More recently, multi-dimensional studies (Fink et al. 2007, 2010; Sim et al. 2010; Kromer et al. 2010) have explored combined helium and carbon detonations in some depth. Among the assumptions and conclusions are: 1) helium ignition will occur as a detonation for almost any value of helium shell mass, including very small ones down to $0.0035 \mathrm{M}_{\odot}$ (Bildsten et al. 2007; Fink et al. 2010); 2) helium detonation will invariably cause detonation of the carbon-oxygen core (Fink et al. 2010); and 3) for low mass helium shells, detonation of carbon in a range of core masses will give a variety of supernova light curves with a widthluminosity relation not unlike that observed for all but the brightest Type Ia supernovae (Sim et al. 2010; Kromer et al. 2010). Bildsten et al. (2007) also refocused attention on the sub-Chandrasekhar mass models, because of the possibility that they might make an observable class of sub-luminous supernovae, "point-one-a"'s. They associated this phenomenon with a particular class of binary system, AM Canum Venaticorum binaries.

These conclusions have far reaching implications and motivate a careful, independent 
evaluation of the problem. While definitive studies will need to be, and now can be done in three-dimensions, this paper explores, in 1D, the physics and parameter space that such calculations will need to examine. Light curves and spectra for a representative sample of our models are calculated and compared with observations. As we shall see, there is still considerable uncertainty regarding the outcome of sub-Chandrasekhar mass models, and more calculations and observations will be needed to determine what really happens.

We begin by discussing the problem set up - the relevant nuclear physics and how accretion is simulated in the code.

\section{Physics and Procedures}

\subsection{Nuclear Physics}

Because of advances in computer technology and reaction data bases, it is now possible to employ more realistic nuclear physics in models for supernovae than was feasible in the 1990's. For this study, we used the "adaptive reaction network" described in Rauscher et al. (2002). This network was used for both energy generation and nucleosynthesis up to the point where a helium detonation was well underway. After that, energy generation was calculated using the usual 19 isotope network (Woosley \& Weaver 1994), but nucleosynthesis continued to be followed using the larger network. One calculation that used the adaptive network throughout showed no appreciable differences with the (cheaper) calculation using the small network for energy generation.

The adaptive network begins with a basic set of 49 nuclei: $n,{ }^{1} \mathrm{H},{ }^{4} \mathrm{He},{ }^{12-14} \mathrm{C},{ }^{14-15} \mathrm{~N}$, ${ }^{15-18} \mathrm{O},{ }^{18-19} \mathrm{~F},{ }^{20-22} \mathrm{Ne},{ }^{21-24} \mathrm{Na},{ }^{23-26} \mathrm{Mg},{ }^{25-28} \mathrm{Al},{ }^{27-32} \mathrm{Si},{ }^{30-33} \mathrm{P}$, and ${ }^{31-36} \mathrm{~S}$. As the calculation proceeds, nuclei are added or subtracted according to rules regarding minimum abundances and projected flows. By the time that the core was freezing out from nuclear statistical equilibrium following the supernova, the reaction network had grown to over 500 nuclei and included species as heavy as ${ }^{96} \mathrm{Ru}$. Nuclei were retained in the network if they had mass fractions greater than $10^{-23}$. Nuclei were added when they were potential products of any reaction on any nucleus in the network with mass fraction greater than $10^{-10}$. In addition to assuring no important nucleus was overlooked, this also allowed the optimal use of CPU and did not waste storage and time on nuclei with trivial abundances. In principle, the network could also shrink with time, but in practice this did not occur. The necessary reaction rates were taken from a variety of sources. See Woosley \& Heger (2007) for a summary. All reaction rates were appropriately corrected for electron screening.

Using the large reaction network for energy generation in the hydrodynamical calcu- 
lation is important because ignition is influenced by heating from the reaction sequence ${ }^{14} \mathrm{~N}\left(\mathrm{e}^{-}, \nu\right){ }^{14} \mathrm{C}(\alpha, \gamma){ }^{18} \mathrm{O}$ (Hashimoto et al. 1986). Since the electron capture is endoergic, this sequence has a threshold density of $5.78 \times 10^{5} \mathrm{~g} \mathrm{~cm}^{-1}$ and occurs at an interesting rate only for helium densities above about $10^{6} \mathrm{~g} \mathrm{~cm}^{-3}$. Such densities occur at the base of the helium, shell for all but the lowest mass CO-dwarfs and the highest accretion rates considered in this paper. As the burning progresses and the temperature rises, the $3 \alpha$ reaction takes over as the dominant source of energy generation, but electron capture is very important, both for altering the neutron-excess of the material (which affects nucleosynthesis) and for determining the location where the runaway ignites. In particular, the depletion of ${ }^{14} \mathrm{~N}$ by electron capture at the base of the accreted helium layer can act to shift the ignition point outwards for helium shells igniting over $2 \times 10^{6} \mathrm{~g} \mathrm{~cm}^{-3}$. The rate for ${ }^{14} \mathrm{~N}\left(\mathrm{e}^{-}, \nu\right)^{14} \mathrm{C}$ here is taken from Hashimoto et al. (1986), as implemented in machine usable form by MartinezPinedo (private communication). The triple-alpha rate is from Caughlan \& Fowler (1988) as adapted for high density by Nomoto et al. (1985) and the ${ }^{14} \mathrm{C}(\alpha, \gamma){ }^{18} \mathrm{O}$ rate is also from Caughlan \& Fowler (1988) as modified by Buchmann (1996) and Woosley \& Heger (2007).

Also important to the ignition and the possibility of detonation is the reaction sequence ${ }^{12} \mathrm{C}(\mathrm{p}, \gamma){ }^{13} \mathrm{~N}(\alpha, \mathrm{p}){ }^{16} \mathrm{O}$, in which the proton plays a catalytic role in accelerating the relatively inefficient ${ }^{12} \mathrm{C}(\alpha, \gamma){ }^{16} \mathrm{O}$ (Shen \& Bildsten 2009). This reaction proceeds on carbon that is either created by pre-explosive helium burning or dredged up from the CO-core. The abundance of free protons can be significant because the composition of the fuel, almost entirely ${ }^{4} \mathrm{He}$ and ${ }^{14} \mathrm{~N}$, lacks any appreciable neutron excess. The relevant reaction rates are well known (Caughlan \& Fowler 1988). We found that this reaction sequence increased the energy generation rate during the runaway, for temperatures near $10^{9} \mathrm{~K}$, by a factor of several. Since detonation involves a race between the burning rate and a sound wave crossing a small region, a change of a factor of three can have very significant consequences.

\subsection{Accretion}

All calculations in this paper were carried out using the Kepler one-dimensional, implicit hydrodynamics code (Weaver, Zimmerman, \& Woosley 1978; Woosley et al.|2002). The grid in Kepler is Lagrangian, so a proper treatment of accretion is challenging (Woosley \& Weaver) 1994). Accretion is simulated here using a surface boundary pressure that gradually increases at a rate corresponding to the weight of accreted matter until a designated mass is reached. A new Lagrangian shell is then added with this mass, a temperature equal to that of the current outer zone, and a density equal to one-half that of the current outer zone. For sufficiently fine zoning, the new zone adjusts promptly to hydrostatic equilibrium. However, 
the newly accreted surface layers of the star are not in thermal equilibrium, so some memory of the the entropy of the accreted matter is retained. In all cases studied here, the entropy of the accreted material was in the range $S / N_{A} k=3$ to 4 , increasing radially outwards. A decreasing entropy gradient would have caused convection. The entropy here is mostly due to the ions. Two runs, with identical zoning, where the density of the accreted shell was increased by a factor of two (Model 10B) had a critical helium shell mass that differed by 4\%. As expected, the calculation with the higher accretion density (lower entropy) ignited later and had the larger mass.

As a consequence of this memory of the accretion entropy, our results are somewhat zoning dependent. With finer surface zoning, the (thinner) outer zone of the star has a higher entropy when in hydrostatic equilibrium. Since the zoning in the present study is finer than in Woosley \& Weaver (1994), the entropies of the accreted shells are higher and, consequently, the critical masses for runaway are smaller, for the same accretion rate and CO-dwarf mass. Smaller zones also mean a shorter time between zone addition and less time for cooling. This too contributes to reducing the critical mass. For example, in the 1994 study, a $0.7 \mathrm{M}_{\odot} \mathrm{CO}$ core accreting at $5 \times 10^{-8} \mathrm{M}_{\odot} \mathrm{y}^{-1}$ accumulated $0.13 \mathrm{M}_{\odot}$ before running away. In the present study, it accretes 0.12 . Previously, a $0.9 \mathrm{M}_{\odot}$ core accreting at $3.5 \times 10^{-8}$ $\mathrm{M}_{\odot} \mathrm{y}^{-1}$ accreted $0.18 \mathrm{M}_{\odot}$ of helium. The same core here, accreting at $3 \times 10^{-8}$ and $4 \times 10^{-8}$ $\mathrm{M}_{\odot} \mathrm{y}^{-1}$, runs away after accreting only 0.13 and $0.11 \mathrm{M}_{\odot}$ respectively.

While this variation is troublesome, the magnitude of the effect is not large compared with that expected if other uncertain parameters of the problem are varied, such as the metallicity (which affects ignition by ${ }^{14} \mathrm{~N}\left(\mathrm{e}^{-}, \nu\right){ }^{14} \mathrm{C}(\alpha, \gamma){ }^{18} \mathrm{O}$ ), the CO-core temperature (i.e., the luminosity) of the accreting $\mathrm{CO}$ dwarf, and time varying accretion rates. The actual

entropy of the accreted material will depend on difficult-to-model radiative efficiencies as the matter accumulates through a shock onto the white dwarf. As a result, the correspondence between accretion rate and critical mass derived here is only approximate. To compensate, we examined a broad range of models. These models might be more appropriately categorized by their CO-dwarf mass, helium shell mass, and location of the ignition than by their accretion rate. At the present time though, there is no compelling reason to rule any of them out.

\subsection{Initial Models}

Initial models were constructed as described in Woosley \& Weaver (1994), except that finer zoning was used, especially near the center of the white dwarf where a detonation might occur, and in the accreting helium shell. We also considered heavier mass CO-cores, higher accretion rates, and hotter white dwarfs than Woosley \& Weaver (1994). These changes 
were motivated by a desire to examine models with smaller helium shell masses at the time of explosion. It will turn out that the higher mass helium shells give supernovae that look less like the Type Ia supernovae (SN Ia) that have been observed so far. We also wanted to compare results with Bildsten et al. (2007) and Sim et al. (2010), who considered heavier CO-cores.

CO-dwarfs with masses of $0.7,0.8,0.9,1.0$ and $1.1 \mathrm{M}_{\odot}$ were allowed to cool and relax into thermal equilibrium with a luminosity of either $0.01 \mathrm{~L}_{\odot}$ or $1.0 \mathrm{~L}_{\odot}$. Cooling to $0.01 \mathrm{~L}_{\odot}$ takes about $200 \mathrm{My}$, while cooling to $1 \mathrm{~L}_{\odot}$, in the absence of accretion only takes about 10 My (Renedo et al. 2010), comparable to the accretion time. White dwarfs with the higher luminosity had typical temperatures in the outer CO-core just beneath the accreting helium of $7-8 \times 10^{7} \mathrm{~K}$ during the accretion. The lower luminosity dwarfs were much cooler with central temperatures $\sim 1-2 \times 10^{7}$ at the onset and the heat generated by accretion flowed more readily into them. The actual value of temperature in these models depended much more on the individual mass, accretion rate, location, and stage of accretion.

Coulomb corrections were included the equation of state. The initial composition of the CO-dwarfs was $49.5 \%{ }^{12} \mathrm{C}, 49.5 \%{ }^{16} \mathrm{O}$, and $1 \%{ }^{22} \mathrm{Ne}$. The composition of the accreted material was $99 \%{ }^{4} \mathrm{He}$ and $1 \%{ }^{14} \mathrm{~N}$, i.e., approximately what is expected for solar metallicity. No elements heavier than ${ }^{22} \mathrm{Ne}$ were included. Once the white dwarf had relaxed to the desired luminosity, set here by a central boundary condition, accretion was initiated at a specified rate and nuclear burning turned on. During the accretion and ignition phases and the early stages of helium detonation, the full reaction network ( $\S 2.1$ ) was coupled to the hydrodynamic calculation. Time-dependent mixing-length convection was included, though no convection occurred until helium burning ignited. The convective speed was calculated according to local (zonal) gradients, but the convective speed was not allowed to exceed $20 \%$ sonic.

The combinations of CO-dwarf mass, accretion rate and white dwarf luminosity studied are summarized in Tables 1 and 2. Models are named according to the mass of the CO-core, times 10, a letter indicating relative accretion rate ("A" or "AA" being the highest accretion rate considered for a given mass), and, in some cases, an " $\mathrm{H}$ " (Table 2) to indicate that the CO-substrate was "hot", i.e., that the initial luminosity of the accreting white dwarf was $1 \mathrm{~L}_{\odot}$, not $0.01 \mathrm{~L}_{\odot}$. A "1" at the end of the model name indicates that detonation of the CO-core was suppressed and only the helium layer exploded.

Helium was added as described in $\S 2.2$. For most of the runs, the mass of each helium zone was $1-2 \times 10^{30} \mathrm{~g}$, though in some studies, especially those with smaller critical helium shell masses, finer, variable mass zones were used. The number of helium zones added is indicated for each model in Tables 1 and 2. As the helium shell grew by accretion, the density 
and temperature at its base increased due to gravitational compression and, eventually, nuclear burning. The helium layer was close to isothermal, but often with a small inverted temperature gradient, that is higher temperatures occurred closer to the surface. The highest temperature was always many zones below the surface though. For example, for a $1.0 \mathrm{M}_{\odot}$ CO-core accreting at $5 \times 10^{-8} \mathrm{M}_{\odot} \mathrm{y}^{-1}$ (Model 10B), the temperature at the base of the helium shell when helium burning first became rapid enough to power convection was $8.55 \times 10^{7} \mathrm{~K}$ and the density there was $1.90 \times 10^{6} \mathrm{~g} \mathrm{~cm}^{-3}$. However the maximum temperature and the base of the convective region was situated $0.026 \mathrm{M}_{\odot}$ farther out where the temperature and density were $9.66 \times 10^{7} \mathrm{~K}$ and $1.35 \times 10^{6} \mathrm{~g} \mathrm{~cm}^{-3}$. As the runaway progressed, the convective region grew and its base moved inwards to $0.020 \mathrm{M}_{\odot}$ above the interface $\left(0.0618 \mathrm{M}_{\odot}\right.$ beneath the surface) as the temperature rose and density declined (Fig. 1).

The ignition characteristics of other CO-core masses and accretion rates included in this study are given in Tables 1 and 2. $\mathrm{M}_{\mathrm{acc}}$ is the mass of helium accreted prior to runaway and $\mathrm{M}_{\mathrm{ign}}$ is the mass, measured inwards from the surface, where the runaway develops. If $\mathrm{M}_{\mathrm{ign}}$ equals $\mathrm{M}_{\mathrm{acc}}$, the runaway develops at the CO-core helium interface. The two densities given, $\rho_{\text {ign }}$ and $\rho_{\text {run }}$, are the values at the base of the accreted helium shell and the location of the runaway. Both densities are evaluated at the time when the maximum power in the convective shell reached $10^{47} \mathrm{erg} \mathrm{s}^{-1}(\S$ 3.1) for those models that detonated. For the models that did not detonate (those indicated with a "d" in the table), the densities were evaluated when the energy generation was a maximum, typically for luminosities between $10^{46} \mathrm{erg} \mathrm{s}^{-1}$ and $10^{47} \mathrm{erg} \mathrm{s}^{-1}$. Additional information concerning ignition conditions is given in Table 3 , which gives the temperature and density at the base of the helium convection zone when the maximum convective luminosity equals the values indicated.

\section{Ignition, Convection and Outcomes}

\subsection{The Freezing Out of Convection}

Convective energy transport and mixing were followed in Kepler using time-dependent

mixing length theory (Weaver, Zimmerman, \& Woosley 1978; Woosley \& Weaver 1988). While this should be adequate during ignition and the early stages of the runaway, mixing length theory certainly breaks down when the burning time scale becomes shorter than the convective turnover time.

Consider again Model 10B. As Fig. 1 shows, as the runaway progresses, the convection zone grows outwards from the ignition region, raising the temperature and lowering the density so as to maintain an approximately adiabatic profile. As the temperature at the 
base of the convective shell rises, the luminosity gradually increases. It is only after about 15 years of convection that the runaway finally culminates in explosion. During this interval there is ample time for many convective turnovers and an adiabatic temperature profile is maintained until close to the end.

Characteristics of the convection can be estimated using mixing length arguments (e.g., Woosley et al. 2004). The convective speed is approximately

$$
v_{\mathrm{rms}} \approx\left(\frac{4 G \delta_{\mathrm{P}} L}{3 c_{\mathrm{P}} T}\right)^{1 / 3}
$$

where $\mathrm{L}$ is a typical luminosity in the convective shell and

$$
\begin{aligned}
\delta_{\mathrm{P}} & =-\left(\frac{\partial \ln \rho}{\partial \ln T}\right) \\
& =-\frac{T}{\rho} \frac{\Delta \rho}{\Delta T} \\
& =\frac{T}{\rho}\left(\frac{\partial \mathrm{P}}{\partial T}\right)_{\rho}\left(\frac{\partial \mathrm{P}}{\partial \rho}\right)_{T}^{-1} \\
& \approx 0.20\left(\frac{T_{8}}{2.5}\right)\left(\frac{1}{\rho_{6}}\right) .
\end{aligned}
$$

Here $T_{8}$ is the temperature in units of $10^{8} \mathrm{~K}, \rho_{6}$, the density in units of $10^{6} \mathrm{~g} \mathrm{~cm}^{-1}$, and $c_{\mathrm{P}}$, the specific heat at constant pressure. The partial derivatives of the pressure can be evaluated using the Helmholtz equation of state (Timmes \& Swesty 2000a). The specific heat varies slowly with temperature and density in the region of interest and has a value $7.2 \times 10^{7} \mathrm{erg} \mathrm{g}^{-1} \mathrm{~K}^{-1}$ for $T_{8}=2.5$ and $\rho_{6}=1$.

The luminosity within the convective shell is not actually a constant, but builds in the energy generating region at the shell's base and declines in the outer regions due to heating and expansion. It has a maximum value though, which is achieved a short distance above the energy generating region, and that will be treated here as representative. When $L_{\max } \sim 10^{45}$ erg, the temperature and density at the base of the convective shell for Model 10B are $2.10 \times 10^{8} \mathrm{~K}$ and $1.34 \times 10^{6} \mathrm{~g} \mathrm{~cm}^{-3}$ respectively. Equation (11) then gives a convective speed of $90 \mathrm{~km} \mathrm{~s}^{-1}$, or about $3 \%$ sonic. The pressure scale height is $340 \mathrm{~km}$, so the time for matter to rise a scale height is about $4 \mathrm{~s}$. The nuclear time scale at this point in Model 10B, the time for the helium to runaway to high temperature if convection were to be abruptly turned off, is $4.1 \mathrm{~s}$, but with convection left on the time scale is considerably longer. The time for a sound wave to go half way round the star at the radius of the convective shell, $3.8 \times 10^{8} \mathrm{~cm}$, is $4.6 \mathrm{~s}$. Since both these time scales are still comparable to the time convection requires to cool the burning region, this might be regarded as the "last good mixing-length convection 
model". The explosion is still developing in an approximately spherically symmetric manner and convection is able to carry away the heat generated by the helium burning reactions.

As the temperature continues to rise however, the luminosity and speeds in the convection zone increase while the nuclear time scale decreases rapidly. Twenty two seconds later (if convection is left on), the maximum luminosity in the convective shell reaches $10^{46} \mathrm{erg}$ $\mathrm{s}^{-1}$ and the temperature at its base is $2.44 \times 10^{8} \mathrm{~K}$. The nuclear time scale, in the absence of convection, has now shrunk to $0.713 \mathrm{~s}$ while the convective speed has only grown to 210 $\mathrm{km} \mathrm{s}^{-1}$, so the convective turnover time is still a second or so (depending upon the choice of the mixing length). Thus the nuclear time scale has become shorter than the convective time scale. This critical temperature, about $2.5-3.0 \times 10^{8} \mathrm{~K}$ for Model 10B, plays the same role in the sub-Chandrasekhar mass models as the better known number, $7 \times 10^{8} \mathrm{~K}$, does in the break down of convection in the standard carbon-deflagration model (Woosley et al. 2004). It will take similar multi-dimensional calculations (Zingale et al. 2009) to follow the subsequent evolution. Certainly by this point though, communication around the star has been lost and the runaway(s) will proceed according to local conditions established at the last good mixing length convection model (see $\S 5.1$ ).

Using mixing length theory beyond this point is clearly perilous, but continuing the calculation to a luminosity of 5,10 , and $50 \times 10^{46} \mathrm{erg} \mathrm{s}^{-1}$ with convection still on, the temperature at the base of the convection zone rises to $2.76 \times 10^{8}, 2.96 \times 10^{8}$, and $3.68 \times$ $10^{8}$ respectively. The corresponding nuclear time scales drop to $0.253,0.149$, and $0.046 \mathrm{~s}$. Meanwhile, the convective speed only increases modestly, roughly as $L^{1 / 3}$. So by the time these latter temperatures are reached, the runaway has certainly become localized to a small fraction of a scale height.

Different outcomes result if the six models with temperature gradients given in Fig. [1 are allowed to evolve with convection turned off. The first three models, one with the ignition conditions (when convection first started to occur) and the two with luminosities 0.1 and $1 \times 10^{46} \mathrm{erg} \mathrm{s}^{-1}$ give rise to deflagrations. The temperature gradient steepens until finally a small region runs away on a time longer than the sound crossing time of the region. The density goes down while the pressure remains constant. A thin shell instability develops with an inverted density. The other three models with luminosities 5, 10 and $50 \times 10^{46} \mathrm{erg}$ $\mathrm{s}^{-1}$ generate outwards moving detonation waves. This result is analogous to that obtained by Woosley (1990) for the usual carbon-deflagration (Chandrasekhar-mass) scenario. There, if convection was halted at a base temperature of $8.5 \times 10^{8} \mathrm{~K}$ a carbon-burning-initiated detonation occurred, but if convection were halted a bit earlier at $8.0 \times 10^{8} \mathrm{~K}$, a deflagration occurred. For the carbon burning case, it is now thought that the deflagration is favored (Zingale et al. 2009). What happens in the helium burning case? 


\subsection{Conditions for Helium Detonation}

Shallow temperature gradients are necessary to initiate a detonation by the Zeldovich mechanism; steep ones inhibit it (Zel'Dovich et al. 1970; Blinnikov \& Khokhlov 1987; Woosley 1990). Convection, so long as it operates efficiently, keeps the temperature gradient adiabatic. At each point on that adiabatic temperature profile there is a nuclear time scale. If there exist regions that are separated by a distance such that the ratio of that distance to the difference in nuclear time scale implies a phase velocity that is supersonic, a detonation can form. On the other hand, if the phase velocity is subsonic, a deflagration results. The extreme non-linearity of the burning rate tends to sharpen temperature gradients and only efficient mixing will suppress the tendency to give birth to deflagrations.

There are three ways of estimating the necessary conditions for helium detonation, each with its own strengths and shortcomings.

First, is the analytic approach. Consider the range of peak temperatures and densities where convection is expected to freeze out in most of our models: $2.0 \lesssim T_{8} \lesssim 3.5$ and $0.5 \lesssim \rho_{6} \lesssim 5$. Table 3 and Fig. 2 show the temperature and density at the base of the convective shell that is running away for the models defined in Tables 1 and 2. The conditions are given at two times that approximately define the limits of mixing length convection - luminosity equals $10^{46}$ and $10^{47} \mathrm{erg} \mathrm{s}^{-1}$.

It is useful to define a nuclear time scale, $\tau_{\text {run }}$, equal to the time required to run away to high temperature, say over $1.2 \times 10^{9} \mathrm{~K}$, starting from the given conditions. This time scale is evaluated here empirically using the models and includes the complications of energy generation by ${ }^{12} \mathrm{C}(\mathrm{p}, \gamma){ }^{13} \mathrm{~N}(\alpha, \mathrm{p})^{16} \mathrm{O}$, as well as the local thermodynamic evolution of the model. That evolution is isobaric until quite close to the end, when the pressure starts to accumulate in those models that detonate. To an accuracy of $50 \%$ in the range of temperature and density given above, the time scale to run away is given by

$$
\tau_{\text {run }} \approx 3.4 \times 10^{-4} \exp \left(20 / T_{8}\right) \rho_{6}^{-2.3} \mathrm{~s} .
$$

An exponential was found to fit the actual values of $\tau_{\text {run }}$ significantly better than a power law, possibly reflecting the temperature sensitivity of the helium burning reaction rate. A necessary condition for detonation is that the absolute value of the temperature gradient approximately satisfy

$$
c_{s} \frac{d T}{d r} \frac{d \tau_{\text {run }}}{d T} \lesssim 1
$$

For the conditions of interest, the sound speed, $c_{s}$, varies from 2300 to $3200 \mathrm{~km} \mathrm{~s}^{-1}$ and an 
appropriate temperature gradient is the adiabatic one,

$$
\begin{aligned}
\left(\frac{d T}{d r}\right)_{\mathrm{ad}} & =\left(1-\frac{1}{\Gamma_{2}}\right) \frac{T}{P} \frac{d P}{d r} \\
& =\left(1-\frac{1}{\Gamma_{2}}\right) \frac{T}{H}
\end{aligned}
$$

where $H=(1 / P d P / d r)^{-1}$, is the pressure scale height. Typically $\mathrm{H} \approx 300 \mathrm{~km}$, and, with $c_{s}=3000 \mathrm{~km} \mathrm{~s}^{-1}, T \approx 3 \times 10^{8} \mathrm{~K}$, and $\Gamma_{2}=3 / 2$, one finds $d T / d r \approx 3 \mathrm{~K} \mathrm{~cm}^{-1}$. This value for the gradient agrees very well with that found in the models that detonated at the times and conditions given in Table 3 . Equation (44) then gives, as a condition for detonation,

$$
\rho_{6} \gtrsim\left(\frac{0.0607}{T_{8}^{2}} \exp \left(20 / T_{8}\right)\right)^{1 / 2.3} \text {. }
$$

This equation, plotted as the topmost solid line in Fig. 2, delineates those regions that are likely to detonate from those that are not. Based solely upon this constraint, none of the models calculated in this paper would detonate. However, several approximations were made in deriving this condition, and eq. (44), itself, is an approximation that depends upon the dimensionality of the problem and equation of state. It also neglects gas dynamic effects. A region close to burning on a sonic time scale will not stay in pressure equilibrium with its surroundings. Higher pressure will shorten the local time scale and expansion of the burning region will alter the temperature gradient in its vicinity. As a result, detonation can happen at a lower density than eq. (77) suggests.

A more accurate estimate of the conditions for detonation in a plane comes from the models themselves. Empirically, all of the models with convective powers of $10^{47} \mathrm{erg} \mathrm{s}^{-1}$ detonated along with a substantial fraction of those with $10^{46} \mathrm{erg} \mathrm{s}^{-1}$. The dashed line in Fig. 2, which is the density given by eq. (7) divided by 4 , is arbitrary, but is a more accurate representation of the model results.

The story does not end here, though, because the detonation probably does not originate in spherical shell (which locally resembles a plane) as these one-dimensional models necessarily assume. A more realistic description is probably detonation starting from a point or set of points $(\S 5.1$ ). To examine the conditions required for detonation starting from a point, spheres of helium of constant density and size were constructed using an approach similar to that employed by Niemeyer \& Woosley (1997) to study carbon detonation. Each sphere, about $100 \mathrm{~km}$ in radius, was zoned into spherical shells, each $1 \mathrm{~km}$ thick. A temperature gradient was imposed such that $d T / d r=3 \mathrm{~K} \mathrm{~cm}^{-1}$ and three choices of central temperature were explored, $2.5,3.0$, and $3.5 \times 10^{8} \mathrm{~K}$. The spheres were evolved with the Kepler code using the large nuclear reaction network and therefore including carbon burning 
by ${ }^{12} \mathrm{C}(\mathrm{p}, \gamma){ }^{13} \mathrm{~N}(\alpha, \mathrm{p}){ }^{16} \mathrm{O}$. The density was varied and the critical value of density required to develop a successful detonation was determined. For example, at $T_{8}=3.5$, a $100 \mathrm{~km}$ sphere with constant density $1.5 \times 10^{6} \mathrm{~g} \mathrm{~cm}^{-3}$ detonated while one with density $1.0 \times 10^{6} \mathrm{~g} \mathrm{~cm}^{-3}$ did not. The resulting points are plotted with error bars as triangles in Fig. 2 ,

The constraints from the numerical detonation experiments agree reasonably well with the curve given by eq. (7), although they lie below it, as expected. The correct answer is obviously dependent upon the actual geometry of the runaway. Is it locally more "point-like" or more like a plane? For now, detonations below $10^{6} \mathrm{~g} \mathrm{~cm}^{-3}$ should be treated with caution (see also Nomoto 1982b), until the defining multi-dimensional calculations of ignition are done.

Also shown in Fig. 2, as the lower solid line, is the condition $\tau_{\text {run }}=2 \mathrm{~s}$, hence

$$
\rho_{\mathrm{defl}, 6} \approx\left(1.68 \times 10^{-4} \exp \left(20 / T_{8}\right)\right)^{1 / 2.3}
$$

Very roughly, models below this line will be able to transport their nuclear energy by convection throughout the explosion and not experience a violent, hydrodynamic event. Between this line and the detonation line, deflagrations occur. Given the uncertainties discussed here, deflagration could happen in anywhere from none to most of our models. Since we are restricted here to one-dimensional simulations, except for a few models with "d" in Table 1 and Table 2, all our simulations experienced helium detonation. Those few that did not were either helium novae or deflagrations.

\subsection{Helium Deflagration}

If convection fails to set up the proper conditions for detonation, but energy is generated at a much faster rate than convection can carry, a deflagration will develop (Fig. 2). The temperature rapidly rises in the ignition region, becoming almost discontinuous and localized to one or a few zones, but the pressure does not change greatly. Consequently the density goes down dramatically. This is the starting point for a deflagration.

In the carbon deflagration model for Chandrasekhar-mass explosions, a thin flame forms at this point and that flame is moved around by the Rayleigh-Taylor instability and turbulence. Here the situation is different, both because the runaway is happening on a shell rather than near the center of a sphere and because a helium burning "flame" is thicker and more subject to disruption by turbulence. The properties of laminar conductive flames in helium are well determined, (Timmes \& Niemeyer 2000b). Typical flame speeds and widths for densities $10^{6}-10^{7} \mathrm{~g} \mathrm{~cm}^{-3}$ are $10^{3}-10^{5} \mathrm{~cm} \mathrm{~s}^{-1}$ and $100-1 \mathrm{~cm}$, respectively. However, 
the flames here are born in a medium that is both turbulent on small scales and characterized by rapid bulk flows on large scales due to the convection. Convective speeds are $\sim 100$ $\mathrm{km} \mathrm{s}^{-1}$ (\$ 3.1) and the pressure scale height is a few hundred $\mathrm{km}$. This convection drives turbulence through shear instabilities on a smaller scale. Judging from the analogous case in carbon deflagration, roughly one order of magnitude in length and velocity scale separate the bulk flow from turbulence that might be described as isotropic with a Kolmogorov spectrum (Zingale et al. 2005; Röpke 2007). Thus, very approximately, we expect the turbulence due to convection at the time the runaway ignites to have a characteristic speed $\sim 10 \mathrm{~km}$ $\mathrm{s}^{-1}$ on an integral scale of about $30 \mathrm{~km}$. Kolmogorov scaling $\left(v \propto l^{1 / 3}\right)$ down to the flame width (e.g., $100 \mathrm{~cm}$ at $10^{6} \mathrm{~g} \mathrm{~cm}^{-3}$ ) then gives a turbulent speed of $\sim 3 \times 10^{4} \mathrm{~cm} \mathrm{~s}^{-1}$, significantly greater than the laminar speed at that density, but still very subsonic. Unlike carbon deflagration, helium deflagration at densities below about $10^{7} \mathrm{~g} \mathrm{~cm}^{-3}$ commences in the distributed burning regime (Timmes \& Niemever 2000b; Shen et al. 2010) where turbulent transport dominates conduction (e.g., Aspden et al. 2008).

However, the rate of helium burning is not determined by the speed of a flame but by the large scale flows in which the burning is embedded (Damköhler 1940). The ash produced by the helium burning has a lower density than the cold helium fuel and floats. Initially, regions of hot ash will form with sizes and geometries determined by the chaotic temperature conditions in the convective flow. These regions will grow as a consequence of the turbulence generated by convection and, later, the turbulence generated by their own rise. For a density contrast near unity and a gravitational acceleration, $g=G M / r^{2} \sim 10^{9} \mathrm{~cm} \mathrm{~s}^{-2}$, hot ash can already reach float speeds of $100 \mathrm{~km} \mathrm{~s}^{-1}$, in a few hundredths of a second. After that, the Rayleigh-Taylor instability, governs the overall progress of the burning while turbulence governs the flame speed on small scales. The problem at that point has clearly become multi-dimensional. We shall attempt to model such events here using a "Sharp-Wheeler" description (Niemeyer \& Woosley 1997) of the burning, but clearly any one-dimensional treatment is very approximate $(\oint 5.3)$.

Two models were explored. One, Model 8DEFL, was based upon Model 8C. Detonation was suppressed by taking the starting conditions to be when the luminosity equaled $10^{45}$ erg $\mathrm{s}^{-1}$ rather than $10^{47} \mathrm{erg} \mathrm{s}^{-1}$. The other, Model 10DEFL, was a recomputation of Model 10D with finer zoning. Because of the finer zoning the runaway ignited at a somewhat reduced critical mass and density ( $\S 2.2$, Table 1). When the nuclear time step had declined to less than $1 \mathrm{~ms}$ in the models, one-dimensional deflagration physics was turned on in Kepler. The clock was zeroed and a flame was propagated according to the prescription

$$
v_{\text {flame }}=\operatorname{Max}\left(A g_{\text {eff }} t, v_{\text {conv }}\right) \text {. }
$$

Here $g_{\text {eff }}$ is the local acceleration due to gravity, $G M(r) / r^{2}$, times the Atwood number. The 
Atwood number measures the density contrast between fuel and ash and is close to unity for helium burning around $10^{6} \mathrm{~g} \mathrm{~cm}^{-3}$. The constant A is approximately $0.12 \pm$ a factor of two (Glimm \& Li 1988). Here, motivated by a desire to have substantial burning in the deflagration, we chose $\mathrm{A}=0.2$. Convection prior to the runaway with speed $v_{\text {conv }} \approx 100$ $\mathrm{km} \mathrm{s}^{-1}$ sets a lower bound to the flame speed. This velocity is measured in the co-moving Lagrangian frame and the rate at which mass is burned is

$$
\dot{M}=4 \pi r^{2} \rho_{\text {fuel }} v_{\text {flame }} \text {. }
$$

For the large gravitational acceleration typical here, $g_{\text {eff }} \sim 10^{9} \mathrm{~cm} \mathrm{~s}^{-2}$, floatation dominates over convection after only $0.01 \mathrm{~s}$. Since the local speed of sound is about $3000 \mathrm{~km} \mathrm{~s}^{-1}$, this equation cannot be used beyond about $1 \mathrm{~s}$, but the explosion was over by then in both models studied. Larger values of "A" result in a delayed transition to detonation which is probably not physical. This is not necessarily to say that no transition to detonation occurs as the flame moves down the density gradient near the surface, only that ascribing this to floating bubbles moving at supersonic speeds is not correct.

\section{Model Results}

\subsection{Helium Detonation}

If carbon detonation is suppressed, either by coarse central zoning or limiting the propagation of the helium detonation into the carbon, then only the helium layer explodes and very little additional matter, if any, is ejected. These models are indicated by a " 1 " at the end of the job name in Tables 1 and 2 and the final remnant masses and kinetic energies are given in Table 4. The kinetic energies range from a few times $10^{49}$ erg to a few $\times 10^{50} \mathrm{erg}$, depending on the mass of material that burns, its initial binding energy, and the nucleosynthetic products. Typical values for the kinetic energy are $\sim 10^{51} \mathrm{M}_{\text {Eject }}$, if $\mathrm{M}_{\text {Eject }}$ is measured in solar masses. This corresponds to an energy per mass of $q \sim 5 \times 10^{17} \mathrm{erg} \mathrm{g}^{-1}$ indicating that not all of the helium burns, and implies a typical ejection speed, $(2 q)^{1 / 2} \approx 10,000 \mathrm{~km}$ $\mathrm{s}^{-1}$. Of course, the peak speed will be higher than the mean. The lowest energy explosions, Models 7A1, 7B1, 8A1, 8HBC1, 9A1, 10A1, 10HC1, 10HCD1, and 11HD1, make almost no

${ }^{56} \mathrm{Ni}$ and will thus be very faint. In fact, all but Model 10HCD1 make very little of anything above ${ }^{44} \mathrm{Ti}$ (Table 5). Some will be very faint indeed. They will not be "point Ia" or even "point zero Ia" supernovae ( $\S 7.2)$.

The rest of the models, 7E1, 8D1, 8E1, 8HC, 9C1, 9E1, 10B1, 10E1, 11E1, and 11F1, all but one derived from the cooler accreting white dwarf, make appreciable ${ }^{56} \mathrm{Ni}$ and will make potentially detectable transients with varying properties ( $\S$ 7.1). Typical abundances 
and speeds are like those in the right hand panels of Fig. 1, Fig. 3 and Fig. 4, though the speeds in the latter two figures have been boosted by the explosion of the CO core.

\subsection{Carbon Detonation}

\subsubsection{Double-Detonation Models}

Most of the models studied here were of the double detonation variety where the helium not only detonated, but touched off a secondary detonation in the CO-core. These were either of the edge-lit variety ("a" in Tables 1 and 2), or detonation ignited by compression at the center of the CO-core $(\S 4.2 .2$, "b" in the tables). Qualitatively, the results were the same. In edge-lit detonations, the whole star detonated before any appreciable expansion of the CO-core occurred. In the compressional detonation, most of the core was only slightly compressed before the CO-core detonated. Detonation of the CO-core assured the complete detonation of any unburned portion of the helium shell on the way out. This degeneracy in results will probably not hold for multi-dimensional simulations of the explosion.

The kinematic results of all the explosions are summarized in Table 4 and the nucleosynthesis will be discussed in $\S$ 6. As expected, the higher mass CO-cores produce more

${ }^{56} \mathrm{Ni}$, both because there is more mass, to burn and because burning goes farther at the higher density. Typical results, as exemplified by Models 7D, 9C, 11C and 11E, are shown in Fig. 3 and Fig. 4. In the higher mass models, most of the core burns to ${ }^{56} \mathrm{Ni}$, leaving only a narrow range of mass and velocity where intermediate mass elements are produced. In the lower mass cores, more intermediate mass elements are made with a broad range of velocities, but the supernova will be faint. Light curves and spectra for these models are discussed in $\S 7.1$.

\subsubsection{Condition for Compressional Detonation of the CO-Core}

For sufficiently fine central zoning, all models in which helium detonated also detonated their CO-core. Is this reasonable?

Convergent spherical and cylindrical shocks have been studied extensively because of their wide application to such disparate topics as bombs, laser pellet implosions, sonoluminescence, and diamond synthesis. The solution depends on the dimensionality of the problem, adiabatic exponent of the gas, and density gradient, but is typically given by a 
"scaling law of the second kind" (Zel'Dovich and Raizer 1966),

$$
R=R_{0}\left(1-\frac{t}{t_{o}}\right)^{\alpha}
$$

where $R_{0}$ is the radius at $\mathrm{t}=0$ and $t_{o}$ is the time when the shock reaches the origin. Typical values of $\alpha$ are 0.7 for spherical geometry and 0.85 for cylindrical geometry (Yousaf 1986, and references therein). As the shock converges to a central point or line, constant density is a reasonable approximation. For $\alpha<1$, this implies a velocity that increases as the shock converges to the center. As the shock velocity increases so does the temperature behind it. Given the singularity implied in eq. (11), the temperature achieved at the center of a calculation with perfect spherical symmetry (e.g., a one-dimensional calculation in radial coordinates) will approach an arbitrarily high value depending on how finely one zones the center of the star.

If the region compressed is large enough that its burning time scale is less than its sound crossing time, a detonation can result. The necessary resolution implies very fine zoning in a Lagrangian stellar evolution code such as Kepler. For models with a thick helium shell, above about $0.1 \mathrm{M}_{\odot}$, a resolution at the center of the star of about $100 \mathrm{~km}$, or about $10^{-4}$ $\mathrm{M}_{\odot}$ sufficed, but for models like $10 \mathrm{HC}$ and $11 \mathrm{HD}$, a central zone smaller than $50 \mathrm{~km}$ was required, or about $10^{-5} \mathrm{M}_{\odot}$. The $\mathrm{CO}$ core was "kicked" by the expansion of the helium layer so as to give speeds behind the inwards moving shock in the range $500 \mathrm{~km} \mathrm{~s}^{-1}$ (low mass helium shell explosions) to $1000 \mathrm{~km} \mathrm{~s}^{-1}$ (high mass shells). Small time steps were taken so as to suppress the damping of such weak shocks by the implicit hydrodynamics of the Kepler code. Successful detonation required a temperature of about $1.6 \times 10^{9} \mathrm{~K}$ in the inner zone, and this in turn required that the compressional speeds be boosted to over $3000 \mathrm{~km} \mathrm{~s}^{-1}$ by the focusing at the stellar center.

While the concentration of $\sim 10^{45}$ erg into $10^{-5} \mathrm{M}_{\odot}$ of the mass does not seem unreasonable, the timing is critical. The sound crossing time for the ignition region is about $10 \mathrm{~ms}$. Compressional waves from a substantial fraction of the star must arrive simultaneously to that tolerance. This happens naturally for ignition with spherical or cylindrical symmetry, but may be more difficult in the case of asymmetric, asynchronous ignition ( $\$$ 5.1).

\subsection{Helium Deflagration}

Typical results for a helium deflagration are shown for Model 10DEFL in Fig. 5 and Table1. Model 10DEFL ejected $0.077 \mathrm{M}_{\odot}$ at speeds up to $6500 \mathrm{~km} \mathrm{~s}^{-1}$, but most of this was unburned helium. Only $9 \times 10^{-5} \mathrm{M}_{\odot}$ of ${ }^{56} \mathrm{Ni}$ was synthesized and the major radioactivities 
were instead ${ }^{44} \mathrm{Ti}\left(0.0025 \mathrm{M}_{\odot}\right),{ }^{48} \mathrm{Cr}\left(0.0063 \mathrm{M}_{\odot}\right)$, and ${ }^{52} \mathrm{Fe}\left(0.0016 \mathrm{M}_{\odot}\right)$. Because of the ${ }^{48} \mathrm{Cr}$ and ${ }^{52} \mathrm{Fe}$, these will be brighter events than classical novae, but because of the lack of ${ }^{56} \mathrm{Ni}$, they will be much fainter than a SN Ia. They are indeed similar to the theoretical predictions of Bildsten et al. (2007), but are a result of deflagration, not detonation. The ${ }^{44} \mathrm{Ti}$ synthesis is also very large and nucleosynthetically interesting. It would not take many of these explosions to make the solar abundance of ${ }^{44} \mathrm{Ca}$ and yet they are faint and may have escaped discovery (see $\S$ 7).

Model 8DEFL was quite similar in outcome. A total mass of $0.141 \mathrm{M}_{\odot}$ was ejected of which $0.114 \mathrm{M}_{\odot}$ was ${ }^{4} \mathrm{He}$. About half of the rest $\left(0.015 \mathrm{M}_{\odot}\right)$ was ${ }^{40} \mathrm{Ca}$ and the masses of radioactivities were ${ }^{44} \mathrm{Ti}, 0.0035 \mathrm{M}_{\odot} ;{ }^{48} \mathrm{Cr}, 0.0037 \mathrm{M}_{\odot} ;{ }^{52} \mathrm{Fe}, 4.5 \times 10^{-4} \mathrm{M}_{\odot} ;$ and ${ }^{56} \mathrm{Ni}$, $6.3 \times 10^{-5} \mathrm{M}_{\odot}$. The total kinetic energy was $3 \times 10^{49} \mathrm{erg}$ with most of the material moving at about $6,000 \mathrm{~km} \mathrm{~s}^{-1}$ (helium) and less (the heavier elements). Very little silicon and sulfur were produced and what was made was in a thin shell. Both the mass and location of the intermediate mass elements would change in a multi-dimensional simulation.

Deflagrations are uniquely able to reach a high burning temperature and yet burn only a small fraction of their mass. This is because the matter ahead of the burning front has time to expand, ultimately putting out the flame. The light curves and spectra of these models are discussed in $\S 7.2$.

\subsection{Helium Novae}

Helium novae occur when a massive CO-dwarf accretes a low mass layer of helium that runs away and transports its energy by convection (Kato \& Hachisu 2003). They may have been observed (Ashok \& Banerjee 2003). Models in Tables 1 and 2 with a "d" that were not deflagrations are able to transport the energy developed in the runaway by convection without exceeding a power of $10^{47} \mathrm{erg} \mathrm{s}^{-1}$. These models were not followed through mass ejection which is likely to occur over an extended period, but they were followed until the density at the base of the convection region had declined more than an order of magnitude and the energy generation had declined substantially.

The net binding energy of a gram of helium in the pre-explosive star varies from $1.3 \times 10^{17}$ $\operatorname{erg~g}^{-1}$ for CO-cores of $0.7 \mathrm{M}_{\odot}$ to $3.9 \times 10^{17} \mathrm{erg} \mathrm{g}^{-1}$ for CO-cores of $1.1 \mathrm{M}_{\odot}$ with thick helium shells. The variation mostly reflects the higher gravitational potential at the edge of more massive CO-cores (which also have a smaller radius). These numbers are to be compared

with the energy that is released by helium burning, $1.33 \times 10^{18} \mathrm{erg} \mathrm{g}^{-1}$ if the helium burns to silicon, and $1.51 \times 10^{18} \mathrm{erg} \mathrm{g}^{-1}$ if it burns to nickel. As with classical novae, only a small 
amount of fuel must burn to eject the accreted layer. The mass is probably ejected as a wind over a long period of time during which the luminosity may not greatly exceed the Eddington value (Iben \& Tutukov 1991; Yoon \& Langer 2004).

Typically the composition of these nova-like explosions is enhanced in elements up to calcium, but not beyond. For the less energetic events, those with the lowest ignition densities, magnesium was an abundant product.

\section{Multi-Dimensional Aspects of Ignition and Burning}

While the models in this paper are one dimensional, the physical conditions and time scales obtained are relevant to predicting the multi-dimensional behavior and defining the parameter space for future work. In this section, we discuss the possibility that the helium runaway ignites neither as a spherically symmetric shell (the 1D limit), nor at a single point, but as something in between, either an extended, but bounded region, or a collection of widely separated points ignited at different times. The necessary conditions for a helium detonation to smoothly transition into an inwards carbon detonation at the CO-core interface are also examined.

\subsection{Multi-Point Ignition?}

Convective energy transport in most of our calculations was halted when the maximum power being transported anywhere in the convective shell passed $10^{47} \mathrm{erg} \mathrm{s}^{-1}(\S 3.1$ ). This value assured detonation in most models. However, this assumption in a one-dimensional model implies a degree of coherency in the burning around the star that is clearly unrealistic. Consider, for example, Model 9C. If convection is halted at $10^{47} \mathrm{erg} \mathrm{s}^{-1}$, a detonation ensues $0.16 \mathrm{~s}$ later. Even if convection is (unrealistically) left on, reaching values of $10^{50} \mathrm{erg} \mathrm{s}^{-1}$, the time until runaway is only extended to $0.44 \mathrm{~s}$. Yet the time for a sound wave $\left(c_{s}=2520\right.$ $\mathrm{km} \mathrm{s}^{-1}$ ) to travel half-way around the star (ignition radius equals $3950 \mathrm{~km}$ ) is $4.9 \mathrm{~s}$. Even a detonation front moving at $12,000 \mathrm{~km} \mathrm{~s}^{-1}$ would take about a second to reach the opposite pole. During that time, the entire helium shell would have already detonated spontaneously. Starting at $10^{46} \mathrm{erg} \mathrm{s}^{-1}$ only helps a little. Runaway occurs after $0.95 \mathrm{~s}$ if convection is halted and $4.3 \mathrm{~s}$ if it is not. None of these one-dimensional simulations is a realistic starting

model for a multi-dimensional study of detonation initiated at a single point (e.g., Fink et al. 2010).

To find a realistic starting model, one must consider the evolution at an earlier time when 
communication among various regions burning around the star first starts to break down. Our arguments here resemble those used by Woosley \& Wallace (1982) and Spitkovsky et al. (2002) to explain the spreading of nuclear burning on neutron stars experiencing Type I x-ray bursts. Unlike Spitkovsky et al. (2002) however, we consider the non-rotating case. Also, there is no flame at this stage and hence no large change in pressure scale height, just a small temperature and pressure contrast between burning regions.

Communication among regions burning at different locations on a gravitational equipotential (to good approximation here, a sphere) occurs by the baroclinic instability. A convective cell with a higher temperature at its base than surrounding cells has a higher entropy. Associated with this higher entropy is a larger scale height which moves the center of mass of the convective region slightly outwards, reducing its weight. Compared with cooler regions farther away, this expansion causes lateral pressure imbalances. At the same gravitational equipotential, the pressure in the hotter convective cell at all locations above the burning shell is higher than its surroundings. As a result, matter spreads outwards from the high entropy cell. Because of the increased scale height, the substrate below, upon which the burning rests, be it helium or carbon and oxygen, also feels reduced weight and rises, bringing up material from beneath to replace what is lost due to the spreading above. If the runaway occurs at the CO-He interface, this instability causes mixing.

To illustrate this idea with some approximate numbers, consider the helium burning shell in Model $9 \mathrm{C}$ at a time $40 \mathrm{~s}$ before the runaway when the maximum convective power is $\sim 10^{45} \mathrm{erg} \mathrm{s}^{-1}$. The temperature, density, pressure, and radius at the base of the convection


time later, when the temperature at the base has increased $5 \%$, the density, pressure and radius of this Lagrangian mass point have changed to $1.416 \times 10^{6} \mathrm{~g} \mathrm{~cm}^{-3}, 5.263 \times 10^{22}$ dyne $\mathrm{cm}^{-2}$, and $3958.1 \mathrm{~km}$ respectively. At constant Lagrangian coordinates the pressure has gone down, but the pressure at this latter radius, $3958.1 \mathrm{~km}$, in the starting model (at $10^{45} \mathrm{erg}$ $\mathrm{s}^{-1}$ ) was $5.259 \times 10^{22}$ dyne $\mathrm{cm}^{-2}$. That is, at a given radius the pressure inside the hot cell has gone up and there is now a pressure differential along a line of constant radius connecting the cold and hot models of $4 \times 10^{19}$ dyne $\mathrm{cm}^{-2}$. The difference is even larger, $1.6 \times 10^{20}$ dyne $\mathrm{cm}^{-2}$, one pressure scale height above the base of the convective region. Examination at other times in the run, $1000 \mathrm{~s}$ and $10 \mathrm{~s}$ before runaway gives similar numbers for the pressure difference because the temperature at the base of the helium convection zone is not varying greatly. Since the differential pressure is, for small changes in temperature, proportional to the size of the temperature fluctuation, we conclude that fluctuations in temperature at the base of the helium burning region, $T_{\mathrm{He}}$ give rise to lateral pressure imbalances, in Model $9 \mathrm{C}$, of order $10^{19}\left(100 \delta T_{\mathrm{He}}\right) / T_{\mathrm{He}}$ dyne $\mathrm{cm}^{-2}$, where $\delta T_{\mathrm{He}}$ is the variation in the temperature at the base of the helium convective shell. In three dimensions, the higher pressure in the hotter 
region would lead to outflows, probably greatest about one pressure scale height above the base. The loss of matter from the convective cell would reduce the pressure upon its base, allowing inflow and upwelling to occur.

Given sufficient time, this mixing keeps regions of the convective shell that are on gravitational equipotentials around the star burning at the same temperature. During the last day before the explosion, however, this thermostat breaks down. A pressure difference of $10^{19}$ dyne $\mathrm{cm}^{-2}$ operating over a distance, $\Delta x$, of $12,000 \mathrm{~km}$, the largest distance separating two points on the spherical shell, at a density of $1.4 \times 10^{6} \mathrm{~g} \mathrm{~cm}^{-3}$, gives an acceleration, $a=\rho^{-1} d P / d x \sim 10^{4} \mathrm{~cm} \mathrm{~s}^{-2}$. A lower bound to the time to spread 12,000 km, given this acceleration, is $\sqrt{2 \Delta x / a} \sim 500 \mathrm{~s}$. This assumes free streaming, as in a wind flowing over the surface. Diffusion in the convecting fluid could take much longer.

Past this point, when the first significant global variations in temperature begin to persist, the surface of the star fractures into a large number of smaller regions whose temperatures evolve independently and can potentially develop large contrasts. The typical scale of such regions is probably a pressure scale height, $\sim 300 \mathrm{~km}$, so there could be hundreds of such cells. How many finally run away within one second of another depends on the size of fluctuations that are imprinted from an earlier era and is very difficult to estimate without a full multi-dimensional simulation. There is no compelling reason at this stage though to assume that there is only one or that the distribution has any special symmetry.

Multiple ignitions would vary in starting time as well as location, so "multi-point asynchronous ignition" might best describe the situation. Numerical studies in 2D of the closely related problem of ignition in classical novae suggest that single-point ignition is unlikely (Glasner et al. 2007; Casanova et al. 2010). On the other hand, some observations of x-ray bursts are consistent with ignition at a single point (Bhattacharyya \& Strohmayer 2006). Recent work on sub-Chandrasekhar mass models for supernovae has frequently assumed single-point ignition in three dimensions (e.g. Sim et al. 2010) or multi-point synchronous ignition in two-dimensions (Fink et al. 2007). Given the inherent cylindrical symmetry of a $2 \mathrm{D}$ calculation where ignition occurs on tori, it would be best to repeat these calculations in three dimensions. Spherical (1D) ignition, single point (2D or 3D) ignition, and toroidal ignition all have a preferred axis of symmetry. Multi-point asynchronous ignition does not and may not so easily lead to detonation of the CO core. García-Senz et al. (1999) studied five point asynchronous ignition in $3 \mathrm{D}$ and found no compressional detonation of the $\mathrm{CO}$ core. However, they did see strong collisions among the various helium detonations that might have ignited a carbon detonation. These calculations need to be repeated with higher resolution and lower mass helium, shells.

Our models show that the inward shocks must converge in a region smaller than 100 
$\mathrm{km}$ for carbon detonation to occur. For the lower helium shell masses of the $10 \mathrm{H}$ series, about $50 \mathrm{~km}$, was required. It seems reasonable that this strong focusing effect will be lost if the ignition is asymmetric by much more than $100 \mathrm{~km}$, e.g., elliptical with a minor axis of $100 \mathrm{~km}$ and a major axis of $300 \mathrm{~km}$. Since the pressure scale height is about $300 \mathrm{~km}$, irregularities in ignition on this scale seem reasonable.

These arguments also suggest a novel mixing mechanism that might be applicable to a broad range of astrophysical problems. In the present context, it suggests that, just as in classical novae, that there probably is appreciable mixing between the CO dwarf and the helium shell prior to runaway, at least for those explosions that ignite at the interface. This mixture of carbon and helium is easier to detonate than either alone (Shen \& Bildsten 2009).

Unless the number of ignition points becomes very large though, most of the burning during the runaway will occur in a helium layer that, except at a few points, has not reached the conditions appropriate for a runaway. The best starting model for a 3D simulation then is a model from Table 1 or 2 for the density at the CO interface indicated, but with a temperature that is cool enough everywhere on the star except the ignition point(s) to assure that a spontaneous runaway does not happen in several seconds.

\subsection{Ignition at Altitude}

It is generally agreed that a helium detonation ignited at a sharp CO-He interface will not successfully propagate into the CO-core (e.g., Livne \& Glasner 1990), while a detonation ignited well above the interface will, if the density is high enough (e.g., Arnett 1997; Benz 1997; García-Senz et al. 1999). Many of our models, those labeled with an "a" in Table 1 and Table 2, ignited their helium sufficiently high above the interface that an inwards as well as outwards detonation was generated. In those cases, the helium detonation transitioned successfully into an inwards moving carbon detonation. Generally, those models had thicker helium shells and colder white dwarf accretors than the others that ignited carbon detonation by focused, star-wide compression. However, detonations are stronger and more robust for nearly planar 1D geometry than for the spherically divergent 3D geometry that actually characterizes ignition at a single point. An unanswered question is how high above the interface helium detonation must begin in order to propagate directly into the carbon. García-Senz et al. (1999) studied this issue numerically, but for a limited choice of (high) densities around $5 \times 10^{6} \mathrm{~g} \mathrm{~cm}^{-3}$. Their results, admittedly with crude zoning, suggested that below $4 \times 10^{6} \mathrm{~g} \mathrm{~cm}^{-3}$, ignition had to occur more than $100 \mathrm{~km}$ above the CO interface in order for the helium detonation to propagate into the carbon. Detonation thus became unlikely below this density. What physics sets this critical density and how robust is the 
constraint? Once again, this is a question that 3D simulation will probably answer soon, but we can make some estimates.

Assume that the helium detonation propagates into the carbon when its momentum is sufficiently large to ignite a "critical mass" of carbon. Then the necessary critical mass of helium must approximately equal or exceed the critical mass of carbon at the same density. Critical masses for carbon detonation have been determined numerically by Röpke et al. (2007), who found a sensitive dependence of the size on density. For a density of $10^{7} \mathrm{~g}$ $\mathrm{cm}^{-3}$, a length scale of $10 \mathrm{~km}$ suffices to detonate carbon, but for $3 \times 10^{6} \mathrm{~g} \mathrm{~cm}^{-3}$ the necessary size climbs to $100 \mathrm{~km}$, i.e, a substantial fraction of a pressure scale height in the present models. This is consistent with García-Senz et al. (1999). At $10^{6} \mathrm{~g} \mathrm{~cm}^{-3}$, it became impossible to detonate the carbon. Many of our "double detonation" models have densities at the $\mathrm{CO}$ interface in the range $1-3 \times 10^{6} \mathrm{~g} \mathrm{~cm}^{-3}$. The $\mathrm{CO}$ detonations in those cases must be regarded as questionable. However, helium detonation is more energetic than carbon detonation and may be facilitated if there is a mixed region of carbon and helium rather than a sharp discontinuity. So this conclusion could be overly pessimistic. Also if "edge-lit" carbon detonation fails, compression of the core or collisions of helium detonations (García-Senz et al. 1999) could still lead to carbon detonation.

\subsection{Deflagration}

If detonation does not occur, then a deflagration of some sort will ensue ( $\$$ 4.3). For those models noted with a "d" in Table 1 and Table 2, convection is able to maintain a nearly adiabatic gradient throughout the runaway and the result resembles an ordinary, though powerful classical nova. All of the other models will develop a strong density inversion and grossly superadiabatic temperature gradients. While a flame in the traditional sense of a nearly discontinuous composition change propagated by conduction (or turbulence) and burning may not develop, the burning rate is greatly in excess of what ordinary convection can carry, yet the speeds are subsonic.

Like the detonation discussed in $\S$ 5.1, we expect the deflagration to be ignited at one or several points scattered around the star. It will not happen simultaneously everywhere on a spherical shell. The propagation of this sort of burning has been discussed by Fryxell \& Woosley (1982) and Spitkovsky et al. (2002). The larger scale height associated with the burning region leads to lateral spreading and mixing of the helium layer. For a ro-

tating white dwarf, Spitkovsky et al. (2002) gives an approximate expression for the burning 
speed around the star,

$$
v_{\text {flame }} \approx \frac{\sqrt{g L}}{\tau_{\text {nuc }} f}
$$

where $L$ is the pressure scale height, $g$, the gravitational acceleration, $\tau_{\text {nuc }}$, some estimate of the burning time scale and $f=2 \Omega \operatorname{Cos}(\theta)$. Here $\Omega$ is the angular rotation rate of the white

dwarf and $\pi / 2-\theta$ is the latitude. Typically the burning time, once a luminosity $\sim 10^{46} \mathrm{erg}$ $\mathrm{s}^{-1}$ is reached, is about a second. Taking a value of $f \sim 1 \mathrm{rad} \mathrm{s}^{-1}$, i.e., moderate rotation, but not near break up, the flame speed is $\sqrt{g L}$, or about $1000 \mathrm{~km} \mathrm{~s}^{-1}$, and the time to go half way round the star, about $10 \mathrm{~s}$. A similar value is obtained from scaling arguments for a non-rotating star. The time for buoyant material to float a scale height is about $\sqrt{L / g}$ and during that time the burning might be expected to also mix with a region of size $\sim L$. This time and length scale gives a speed of approximately $\sqrt{g L}$.

In our one-dimensional models we used a Sharp-Wheeler scaling law for the flame speed $(\S 4.3), v_{\text {flame }}=0.2 g_{\text {eff }} t$. Except for the uncertain number out front, this is also $\sqrt{g L}$ as can be seen by substituting $t=\sqrt{L / g}$ in the Sharp-Wheeler expression.

\section{Nucleosynthesis}

\subsection{Nucleosynthesis in the Models}

The nucleosynthesis of all models is summarized in Tables 5 - 10. Table 5 gives the abundances of the most interesting radioactivities. Table 6 and Table 7 give the "production factors" for major species in those models where both the helium shell and carbon-oxygen core detonate. For these stars that completely explode, the production factor is defined as the ratio of the overall mass fraction of the given species in the exploded star compared with its mass fraction in the sun. The actual mass produced as a given species is thus this factor times the mass of the whole star times the mass fraction in the sun as given by Lodders (2003). Since the production factors for iron are in the range 200 to 700, production factors less than 10 are considered unimportant and are not given. The stars in Tables 8 and 10 leave a white dwarf remnant. Nevertheless, so long as we confine our attention to species that exist only in the ejecta, the definition of production factor is similar - the average mass fraction in the ejecta plus remnant white dwarf compared to the solar value. This is certainly true of all the spcies we are interested in, namely those heavier than neon. So the mass ejected is again the mass of the star (white dwarf plus helium shell; Table 1) times the yield factor times the solar mass fraction. For progenitors of solar metallicity, the yield factor is thus the mass produced as a given species divided by the mass of that species in the star before the explosion. Because the tables start at silicon, all species given were produced 
in the explosion. Since no $r$-process occurs here and because no initial abundances heavier than ${ }^{22} \mathrm{Ne}$ were included in the calculation, nucleosynthesis above the iron group is not very well represented. An exception is a number of "p-process" isotopes up to about A $=80$ that are produced by alpha capture in the detonation of the helium shell.

The yields of trace isotopes above mass 40, except for ${ }^{56} \mathrm{Ni}$, are also quite uncertain since they depend on uncertain reaction rates for radioactive targets (Table 11). The relevant cross sections have not been measured in the laboratory, but come from theoretical estimates which may be particularly uncertain for self-conjugate $(\mathrm{Z}=\mathrm{N})$ nuclei. In many cases, the yields also depend upon the metallicity of the star and accreted material. Solar values have been assumed here, but that need not always be the case. These uncertainties may underlie the large yields of nickel isotopes in all models and of ${ }^{48} \mathrm{Ti}$ in the helium detonations and deflagrations (Table 8 and Table 10). These yields could easily be off by a factor of two or more.

In general, the nucleosynthesis in those models where both helium and carbon detonate resemble closely that reported previously in Woosley \& Weaver (1994). Substantial amounts of ${ }^{43,44} \mathrm{Ca},{ }^{46,47,48} \mathrm{Ti},{ }^{51} \mathrm{~V},{ }^{50,52,53} \mathrm{Cr},{ }^{55} \mathrm{Mn},{ }^{56,57} \mathrm{Fe},{ }^{58,60,61,62} \mathrm{Ni},{ }^{63,65} \mathrm{Cu}$, and ${ }^{64} \mathrm{Zn}$ are made. Especially large are the productions of ${ }^{44} \mathrm{Ca},{ }^{48} \mathrm{Ti}$ and ${ }^{52} \mathrm{Cr}$ made as ${ }^{44} \mathrm{Ti},{ }^{48} \mathrm{Cr}$ and ${ }^{52}$ Fe respectively. The iron group is also produced in substantial abundances, including of course ${ }^{56} \mathrm{Fe}$ made as ${ }^{56} \mathrm{Ni}$. Given the appreciable uncertainty in the cross sections discussed above, the overall yield pattern is reasonably close to solar, and not markedly inferior to that made in Chandrasekhar-mass explosions (Iwamoto et al. 1999). In fact, the reduced production of ${ }^{58} \mathrm{Ni}$ and production instead of ${ }^{44} \mathrm{Ca},{ }^{47} \mathrm{Ti},{ }^{63,65} \mathrm{Cu}$ and ${ }^{64} \mathrm{Zn}$ are significant improvements. Cobalt and manganese are somewhat deficient however. The species ${ }^{44} \mathrm{Ca}$ is particularly interesting because it is not made in the Chandrasekhar mass models and is inadequately produced in core-collapse supernovae (Timmes et al. 1996). It is also inadequately produced for the higher mass double detonations here (Table 6), but is made sufficiently in the lower mass ones (Table 7). Overall though, it is difficult to make both a solar ratio for ${ }^{44} \mathrm{Ca} /{ }^{56} \mathrm{Fe}$ and sufficient ${ }^{56} \mathrm{Ni}$ to give a bright Type Ia supernova (though Model 10B may be an exception).

The rest of the models, where the carbon does not detonate, produce too little ${ }^{56} \mathrm{Ni}$ to be typical SN Ia. Still, their nucleosynthesis is interesting. The single (helium) detonation models produce many of the same species as the double detonations nut production of the iron group is greatly diminished (Table 8). The largest yields are for $\alpha$-particle nuclei above ${ }^{40} \mathrm{Ca}$ and their neighbors. After decay (Table 11) these make ${ }^{43,44} \mathrm{Ca},{ }^{46,47,48} \mathrm{Ti},{ }^{51} \mathrm{~V},{ }^{52} \mathrm{Cr}$, ${ }^{60,61,62} \mathrm{Ni},{ }^{64} \mathrm{Zn}$, and interesting amounts of ${ }^{68} \mathrm{Zn}{ }^{74} \mathrm{Se}$, and ${ }^{78} \mathrm{Kr}$. While the ${ }^{44} \mathrm{Ca}$ yields are now sufficiently high compared with ${ }^{56} \mathrm{Fe}$, depending on the specific model and uncertain 
reaction rates, they are still low compared with other trace species like ${ }^{48} \mathrm{Ti}$ and ${ }^{61} \mathrm{Ni}$.

Some of the most interesting and most uncertain nucleosynthesis happens in the helium deflagration models (Table 10). Lines from stable ${ }^{40} \mathrm{Ca}$ would be very prominent in the spectrum of this sort of explosion. In some models, ${ }^{44} \mathrm{Ca}$ is the largest nucleosynthetic production. Lacking in ${ }^{56} \mathrm{Ni}$ and having low abundances of other short-lived radioactivities (Table 5), these would be very faint supernovae and might happen frequently. It is important when comparing the resulting light curves and observations to follow the radioactive decays. Thus while ${ }^{44} \mathrm{Ca}$ is an abundant nucleosynthetic product, it is $\mathrm{Ti}$ that will be abundant in the spectrum. The large production of ${ }^{48} \mathrm{Ti}$ reflects synthesis as ${ }^{48} \mathrm{Cr}$. This nucleus decays rapidly to ${ }^{48} \mathrm{~V}$, but the half-life of ${ }^{48} \mathrm{Va}$ is 16 days, so vanadium lines should be prominent in the peak light spectrum. The nucleus ${ }^{45} \mathrm{Sc}$ is made as ${ }^{45} \mathrm{Ti}$, but this decays to ${ }^{45} \mathrm{Sc}$ in a few hours, so the large production of ${ }^{45} \mathrm{Sc}$ should give prominent lines of the element scandium. Analysis of the network flows shows that ${ }^{45} \mathrm{Ti}$ is made by a variety of reactions during explosive helium burning. Chief among them are ${ }^{41} \mathrm{Ca}(\alpha, \gamma){ }^{45} \mathrm{Ti},{ }^{43} \mathrm{Ca}(\mathrm{p}, \gamma){ }^{44} \mathrm{Sc}(\mathrm{p}, \gamma){ }^{45} \mathrm{Ti}$, and ${ }^{44} \mathrm{Ti}(\mathrm{n}, \gamma){ }^{45} \mathrm{Ti}$. The ${ }^{45} \mathrm{Ti}$ is destroyed by ${ }^{45} \mathrm{Ti}(\mathrm{p}, \gamma)^{46} \mathrm{~V}$. Its sysnthesis thus depends on uncertain nuclear physics like the ${ }^{44} \mathrm{Sc}$ and ${ }^{44,45} \mathrm{Ti}$ cross sections, as well as the neutron excess.

The large production of ${ }^{44} \mathrm{Ti}$ (half-life 60 years) also has implications for the late time supernova light curve. The large productions indicated could keep the supernova shining at $\sim 10^{38} \mathrm{erg} \mathrm{s}^{-1}$ for a century or more (Timmes et al. 1996).

None of the three classes of models discussed - double detonation, helium detonation and helium deflagration - are presently mutually exclusive. SN Ia could possibly come from big dwarfs detonating their cores (if the light curves and spectra were acceptable) and ${ }^{44} \mathrm{Ti}$ from helium deflagrations. For now, there is no nucleosynthetic reason to exclude any of the subChandrasekhar mass models; indeed there is some advantage to having their contribution.

\subsection{Comparison with Previous Results}

As noted above, the nucleosynthesis calculated here, for those models where the helium detonates, agrees well with previous one-dimensional studies of this class of model. It differs appreciably, however, with that found in more recent multi-dimensional studies by Fink et al. (2010) and Kromer et al. (2010). These simulations found far less production of ${ }^{56} \mathrm{Ni}$ and production instead of intermediate mass radioactivities like ${ }^{44} \mathrm{Ti},{ }^{48} \mathrm{Cr}$, and ${ }^{52} \mathrm{Fe}$. In fact, the nucleosynthesis for their detonations resembles that coming from our deflagrations. An interesting comparison is Model 1 of (Fink et al. 2010) and our Model 8B. Both began as 
$0.8 \mathrm{M}_{\odot}$ CO-cores (0.81 for the Fink model) that accreted $0.13-0.14 \mathrm{M}_{\odot}$ of helium and exploded. The mass of the two stars when they exploded was nearly identical, $0.936 \mathrm{M}_{\odot}$ for their Model 1, $0.941 \mathrm{M}_{\odot}$ for our Model 8B, yet detonation of the helium shell in their Model 1 made $8.4 \times 10^{-4} \mathrm{M}_{\odot}$ of ${ }^{56} \mathrm{Ni}$, while Model $8 \mathrm{~B}$ made $0.046 \mathrm{M}_{\odot}$ of ${ }^{56} \mathrm{Ni}$. Why are the two results so different?

There are two reasons. One is the inherent hydrodynamical difference between detonation in a spherical shell (our case) and a sliding detonation that moves around the star at the He-CO-core interface (theirs). In the latter, which should be more realistic, pressure is lost from behind the detonation because the material has an open direction for expansion. This reduces the pressure and temperature in the detonation front and the helium burning reactions do not proceed as far.

A larger effect, however, probably arises from the difference in density at the base of the helium shell in the two initial models: $3.7 \times 10^{5} \mathrm{~g} \mathrm{~cm}^{-3}$ in their Model 1 and $1.55 \times 10^{6}$ $\mathrm{g} \mathrm{cm}^{-3}$ in our Model 8B (the ignition density in Table 1 is less for Model 8B because ignition actually occurs further out in the star). Detonation at the larger density gives higher temperatures and heavier elements. But then why are the densities so different? The assumptions underlying Model 8B have been presented in this paper.

Model 1 of Fink et al. (2010), on the other hand, is derived from a construction provided to them by Bildsten et al. (2007). In that construction, the helium envelope was assumed to be completely convective, i.e., adiabatic, with a temperature at its base (over $6 \times 10^{8}$ $\mathrm{K}$ ) adequate to cause helium to burn on a hydrodynamic time scale. As we have discussed in $\S$ 3.1, the more correct assumption is that convection freezes out when the nuclear time scale is comparable to the convective turnover time. Since convection at this point is still very subsonic, a lower temperature is implied. This makes physical sense and has been demonstrated to be the correct assumption for carbon ignition in Chandrasekhar mass models for SN Ia several times (e.g. Zingale et al. 2009). Consequently our temperatures at the base of helium convective shells are in the range $2-3 \times 10^{8} \mathrm{~K}$, not $6 \times 10^{8} \mathrm{~K}$. As the runaway proceeds, $6 \times 10^{8} \mathrm{~K}$ is eventually reached in our models, but only in a small region. In multi-dimensional simulations, the region will probably be smaller still, on just one side of the star. This high temperature is not a characteristic value at the base of a spherically symmetric, adiabatic envelope. A lower entropy in the helium shell means that, with a similar gravitational potential at the base, it would have a higher density. The difference probably accounts for the discrepancy in density in the two models.

The best result will be achieved when our present pre-supernova models are exploded in three-dimensions. That can be done. For now though, we believe our nucleosynthesis, though one-dimensional, is closer to correct. Table 3 of Fink et al. (2010) shows a rapid rise 
in the fraction of helium that burns to ${ }^{56} \mathrm{Ni}$ as the density approaches $10^{6} \mathrm{~g} \mathrm{~cm}^{-3}$ even in the multi-dimensional sliding-detonation models. We find it difficult to detonate helium in models where the density is as low as $3.7 \times 10^{5} \mathrm{~g} \mathrm{~cm}^{-1}$.

\section{Light Curves and Spectra}

We calculated synthetic light curves and spectra of a representative subset of our models using the time dependent radiation transport code SEDONA (Kasen et al. 2006). The code parameters were similar to those described in previous transport calculations for SNe Ia (e.g., Kasen et al. 2009). The radioactive decay chain of ${ }^{56} \mathrm{Ni}$ was included as well as those of ${ }^{52} \mathrm{Fe}$ and ${ }^{48} \mathrm{Cr}$, along with a gamma-ray transport scheme to determine the energy deposition. Local thermodynamics equilibrium (LTE) was assumed to determine the ionization and excitation state of the ejecta. While often a good approximation for SNe Ia in the early phases, LTE breaks down at later times when nebular line emission and non-thermal ionization effects become significant.

The light curves of the models show considerable diversity in brightnesses and duration as illustrated in Figure 6. The fundamental observable properties of the entire set of model light curves and spectra are summarized in Figures 7,[10. We divide our discussion into those models in which the entire star (both shell and core) explode, and those in which only the helium shell is disrupted.

\section{1. $\quad$ Light Curves and Spectra - Full Star Explosions}

Double detonation explosions of sub-Chandrasekhar white dwarfs were investigated some time ago as possible models for normal SNe Ia (Woosley \& Weaver 1994; Livne \& Arnett 1995) but the idea eventually fell out of favor when calculations of model spectra did not match those of observed events (Hoeflich \& Khokhlov 1996; Nugent et al. 1997). In particular, the spectral features of intermediate mass elements (IMEs) were too weak in the models compared to observations, and the continuum was too blue. More recent, multi-dimensional calculations confirmed the weakness of IME features, but suggested that the models were in fact too red compared to observations, especially after maximum light (Kromer et al. 2010). In either case, the spectral peculiarities are due to the outer shell of radioactive material produced in the detonation of the helium layer. If the helium layer is omitted from the model altogether, the light curves and spectra of detonated bare sub-Chandrasekhar CO

white dwarfs actually agree quite well with observations (Sim et al. 2010). The mass of 
burned material in the outer shell is therefore crucial to the predicted observables.

We find that the models in our set which assumed a "cold" white dwarf (i.e., one with initial luminosity of $0.01 \mathrm{~L}_{\odot}$ ) generally have maximum light spectra that do not resemble observed normal SNe Ia. Figure 11] shows as a typical example the maximum light spectra of Model 9C. The IME absorption features (Si II, S II, Ca II) in the model are too weak to match the normal Type Ia SN2003du, and overall the model more closely resembles the spectroscopically peculiar SN 1991T (although the model lacks the two prominent Fe III lines near $4300 \AA$ and $5000 \AA$ seen in SN 1991T). At later times $(\sim 7$ days past peak) weak IME lines do begin to appear and the spectrum starts to look more normal (Fig. 12). In this sense, the early spectral evolution of these models resembles the class of SN 1999aa-like supernovae (Li et al. 2001), which appear to be intermediate between the normal and spectroscopically peculiar SNe Ia. At yet later times $(\sim 2$ weeks after peak) the line blanketing from iron group elements in the outer layers becomes strong, leading to a very red spectrum, in contrast to what is typically observed in either the normal or the SN 1999aa-like SNe Ia. A similar spectral evolution characterizes most of the "cold" models we calculated, except those with the smallest accreted shell masses. The observational counterpart to the bulk of these models is unclear.

The weakness of the IME features in these models is due to several effects. First, the radioactive heating from the shell serves to further ionize Si II, S II and Ca II, thereby reducing their opacity. Second, the absorption features are diluted because a portion of the continuum luminosity is being generated in the radioactive shell above the IME line forming region. A third reason why the IME features are weak in these models has to do with the dynamical effects of the helium shell on the velocity of ejected IMEs. Regardless of whether the outer layers burn to heavier elements or not, the mass in the shell serves to decelerate the detonated core, thereby limiting the maximum velocity of the IME ejected in the CO detonation. This effect confines the IME to rather narrow shell of velocity range 11,000-13,000 $\mathrm{km} \mathrm{s}^{-1}$. This is in contrast to observed SNe Ia (as well as standard delayeddetonation models) in which the IME velocities typically span a range $9,000-15,000 \mathrm{~km} \mathrm{~s}^{-1}$. The thinness of the IME layer in the present models restricts the geometrical area over which this material covers the photosphere, reducing the strength of the features. Thus, even if we turn off (by hand) the radioactivity in the outer shell, the IME features still remain too weak to reproduce observations (Fig. 12). In principle, Raleigh-Taylor instabilities at the ejectashell interface could broaden the radial distribution of IMEs and increase the depth of the absorption features, an effect not captured in these 1D simulations. In addition, the structure

of the ejecta can be different and aspherical in multi-dimensional models. Fink et al. (2010) show that when the detonation of the helium shell occurs at a point, IMEs are ejected at significantly higher velocities on one side of the ejecta while they are confined to a narrow 
velocity range on the other.

In order to systematically explore the effects of the outer helium shell on the observables, we compare, in Figure 13, the spectra of four models which had an identical core mass of $1 \mathrm{M}_{\odot}$, but which varied in the amount of material which had accreted into the helium shell at the time of detonation. Of these models, $10 \mathrm{HC}$ had the smallest accreted mass: $M_{\text {acc }}=0.0445 \mathrm{M}_{\odot}$ within which was produced only $8 \times 10^{-5} \mathrm{M}_{\odot}$ of ${ }^{56} \mathrm{Ni}$. The maximum light spectrum of this model shows moderately strong IME lines which are in reasonable agreement with those observed in normal SNe Ia (Fig. 14). Model 10A, with a slightly larger helium shell $\left(M_{\text {acc }}=0.064 \mathrm{M}_{\odot}\right)$ also shows some IME absorption features, albeit weaker than what is typically observed. Models $10 \mathrm{~B}$ and $10 \mathrm{D}$, which had yet larger accreted masses, do not show any IME absorptions at all.

We conclude that the spectra of some double detonation models do in fact resemble normal SNe Ia, but only if the accreted mass is small, $M_{\text {acc }} \lesssim 0.05 \mathrm{M}_{\odot}$, and only if a small amount of this is burned to radioactive material. In the present model set, this was only regularly achieved for models in which the white dwarf was "hot" i.e., had an initial luminosity of $1 \mathrm{~L}_{\odot}$. Fig. 15 shows the spectra of a four such "hot" models which varied in the initial white dwarf mass and consequently the amount of ${ }^{56} \mathrm{Ni}$ produced in the core detonation. All of these models have normal looking spectra. In the case of the "cold" white dwarfs $\left(L=0.01 \mathrm{~L}_{\odot}\right)$, on the other hand, the star accretes a larger mass before the helium shell explodes, and the maximum light spectrum generally appears peculiar, as seen for Model 9C (Fig. 13] and [12).

The presence of the outer shell of burned material also impacts the light curves of the models. Figure 16] shows two separate light curve calculations for Model 9C, a typical full star explosion with a "cold" white dwarf. In the first calculation, we included the outer shell of material, while in the second, that shell was removed after explosion - i.e., once the ejecta had reached the homologous expansion phase. The calculations illustrate two main effects of the outer shell on the observables: (1) At and before peak, the decay of ${ }^{56} \mathrm{Ni}$ and other radioactive isotopes in the shell leads to additional heating at the surface, causing the light curves to be slightly brighter and bluer at peak. (2) A week or two after peak, the iron group material in the shell begins to cool to a singly ionized state, which increases the line opacity in the blue wavelength bands. Fluorescence in these lines redistributes flux from shorter to longer wavelengths (Pinto \& Eastman 2000; Kasen 2006), causing the colors to become progressively redder after peak, In the multi-dimensional models studied by Kromer et al. (2010), iron group elements are ejected at significantly higher velocities on one side of the ejecta, causing these line blanketing effects to set in even earlier (for certain viewing angles). In our models, the outer shell results in a faster decline in the U- and B-band light curves after 
peak, leading to a poor (although not terrible) fit to the observed light curve of the normal Type Ia SN 2003du (Stanishev et al. 2007). The overall fit to observations is significantly improved when the outer shell is removed.

The light curves of other full star explosions show similar behavior to that described above, but vary in brightness depending on the ${ }^{56} \mathrm{Ni}$ mass produced in the explosion. The B-band peak magnitudes range from -18.3 to -19.6 and the B-band decline rates from $\Delta M_{15}(B)=0.7-2.5$ mag. Figure 7 shows that the set of models obeys a rather tight widthluminosity relation, however the offset and slope of models with "cold" white dwarfs are not in good agreement with recent observed values for the relation (Folatelli et al. 2010). Systematically, the models decline too rapidly for a given brightness, a consequence of the presence of the burned helium shell. The models with "hot" white dwarfs are in somewhat better agreement with the normalization of the observed WLR, but have too steep a slope, with the decline rate changing by only a small amount $(0.2 \mathrm{mag})$ for a $\sim 1$ mag change in peak B-band magnitude.

\subsection{Light Curves and Spectra - Helium Shell Deflagration and Detonation Models}

The models in which only the helium shell explodes (leaving the CO core intact) produce fainter and more rapidly evolving transients due to the smaller ejecta masses. The observables of helium shell detonations ("Ia" explosions) have been studied previously by Shen et al. (2010). We explore here a wider range of models which show diversity in both their peak magnitudes and spectroscopic properties. The helium shell deflagrations, which involve lower explosion energies than the detonations, have distinct light curves and spectra and are explored for the first time here.

For models assuming initially "cold" white dwarfs, the helium shell detonations produce moderate quantities of ${ }^{56} \mathrm{Ni}$, which powers the light curves. Figure8 8 shows that within this set of models, the B-band peak magnitudes vary from - 15 to -18.3. The B-band rise times range from 4 to 9 days, and correlate tightly with the brightness. This is because the more massive shells both have a longer diffusion time and typically produce a larger mass of radioactive isotopes. The decline rate of the B-band light curves varies from rapid $\left(\Delta M_{15}(B)=2 \mathrm{mag}\right)$ to extremely rapid $\left(\Delta M_{15}(B)=7 \mathrm{mag}\right)$. These fast decline rates reflect not only the low ejecta mass, but also the strong evolution of the colors to the red as the ejecta cool over time. The photospheric velocities of the helium shell detonations are always around 7,000$10,000 \mathrm{~km} \mathrm{~s}^{-1}$ at peak, and the spectral features are broad, with absorptions extending to $\sim 15,000 \mathrm{~km} \mathrm{~s}^{-1}$ (Figure 17). The spectra of models which assumed an initially "cold" 
white dwarf generally lack strong IME absorptions of Si II and S II, and instead show broad features of titanium and iron group elements, similar to those studied in Shen et al. (2010).

The models which assumed an initially "hot" white dwarf generally produce less ${ }^{56} \mathrm{Ni}$ in the shell explosion and greater abundance of IMEs. In this case, radioactive ${ }^{48} \mathrm{Cr}$ becomes the significant source of heating. Fig. 18 shows the light curves and spectra of Model 8HBC1, which ejected $0.097 \mathrm{M}_{\odot}$ of ejecta, but only $1.5 \times 10^{-4} \mathrm{M}_{\odot}$ of ${ }^{56} \mathrm{Ni}$, along with $9 \times 10^{-4} \mathrm{M}_{\odot}$ of ${ }^{48} \mathrm{Cr}$. An additional $2.5 \times 10^{-4}$ of ${ }^{52} \mathrm{Fe}$ was produced, but given the short decay time of this isotope and its daughter, ${ }^{52} \mathrm{Mn}$, it does not contribute significantly to powering the observed transient. The B-band light curve of $8 \mathrm{HBC} 1$ peaks in only 3 days at a remarkably dim magnitude of -13.5. The maximum light spectrum shows significant absorptions due to silicon and sulfur, and overall resembles the spectra of observed subluminous SNe Ia such as SN 1991bg. This is in contrast to the shell explosions of the "cold" white dwarf models, which lacked these features.

The two helium shell deflagration models calculated here (8DEFL and 10DEFL) show dramatically different properties than the helium detonations. Since the total mass of ${ }^{56} \mathrm{Ni}$ is negligible, the light curves are powered primarily by the decay of ${ }^{48} \mathrm{Cr}$. Both models are extremely dim (B-band peak magnitudes of -14.5 and -15) though in principle deflagration of more or less massive shells could produce brighter or dimmer events as well. Unfortunately, we were unable to obtain stable numerical models of thick helium shell deflagration using our simple one-dimensional treatment of the burning. Burning in the deflagration models is mostly incomplete and a significant percentage of helium remains. The explosion energy per unit ejected mass is thus low, which leads to a longer rise time and slower decline rate relative to a detonation model of the same brightness. The photospheric velocities in the deflagration models are very low $\left(\sim 4000 \mathrm{~km} \mathrm{~s}^{-1}\right.$ at peak $)$ and the spectra are characterized by numerous narrow absorption features (Figure 17). Many of the lines are from species not commonly seen in SNe Ia, including features from Sc II. Helium lines are not seen in the synthetic spectra, however it is well known that these lines are extremely sensitive to nonthermal excitation effects due to radioactive decay products. Thus, non-LTE calculations will be needed to predict the actual strength of the helium lines.

Recent observations have revealed a diverse class of dim, brief transients which have occasionally been linked to helium shell explosions. These observed events show a variety of properties. In some cases, for example, the observed photospheric velocities are relatively high and the light curve declines very rapidly (e.g., SN 2002bj Poznanski et al. (2010), SN 2005E Perets et al. (2010), SN2010X Kasliwal et al. (2010)). These properties generally resemble the helium detonation models, however a detailed case-by-case spectral comparison is needed before a firm link can be drawn. Most of the models do predict a a significant 
Ca II IR triplet feature, which is observed in many events, while only a subset of the models predict the Si II and S II features that are occasionally seen. On the other hand, many of the observed events show absorption features of carbon and oxygen, which are not seen in our models, While the $1 \mathrm{D}$ model do not ejecta a significant amount of $\mathrm{C} / \mathrm{O}$, it is possible that in more realistic multi-D calculation some $\mathrm{C} / \mathrm{O}$ may be dredged up from the core and mixed into the ejected shell material.

In other observed peculiar SNe, the spectral features have very low velocities which

are inconsistent with the helium detonations (e.g., SN 2008ha Foley et al. (2009)). It is interesting to suggest that these low velocity events may represent helium shell deflagrations. More detailed model calculations will be needed to clarify the situation. In particular, the 1-D models do not properly capture the large scale mixing that is characteristic of realistic 3-D deflagration burning.

\section{Conclusions}

We have studied one-dimensional models for the explosion of sub-Chandrasekhar mass white dwarfs accreting helium in a binary system. The models included white dwarfs in the mass range 0.7 to $1.1 \mathrm{M}_{\odot}$ that, at the onset of accretion, had luminosities of either 0.01 or 1 solar luminosities, models denoted here as "cold" or "hot". The accretion was followed, as well as the convective phase leading up to the explosion. Depending upon the relative magnitudes of the nuclear, convective, and sonic time scales at the time when energy generation reaches a maximum ( $(3$ ) , four possible outcomes were found and explored. 1) A runaway in which convection carries the energy from start to end, an outcome that resembles classical novae, though with much greater mass ejected. 2) Helium deflagration, in which rapid subsonic burning creates a density inversion at the base of the convective helium shell leading to rapid mixing and burning, also ejecting only the helium shell and a bit of dredged up carbon. 3) Helium shell detonation that leaves behind a hot, but intact CO dwarf. 4) Detonation of the helium shell and of the CO core, either by compression of the latter or direct propagation of the helium detonation into the core. Representative examples of all these outcomes can be found in Table 1 and Table 2. The nucleosynthesis of all models was calculated and the light curves and spectra of many. Possible multi-dimensional modifications of our results were discussed in $\S 5$.

We find, in agreement with Bildsten et al. (2007) and Shen \& Bildsten (2009), a minimum helium shell mass for detonation of $\mathrm{M}_{\min } \approx 0.025 \mathrm{M}_{\odot}$ (Table 22) for a $1.1 \mathrm{M}_{\odot}$ white dwarf with a high crustal temperature. This is substantially less than found in earlier studies (Woosley \& Weaver 1994) because of the use of finer zoning during the accretion, which al- 
ters the entropy of the accreted material ( $\$ 2.2$; up to $40 \%$ change in $\mathrm{M}_{\min }$ ); the inclusion, in the energy generation rate, of new reaction sequences for burning helium and carbon $(\S 2.1$, a factor of two decrease in $\mathrm{M}_{\min }$ ); and the inclusion, in the study, of hotter, higher mass CO-cores (an additional factor of two decrease in $\mathrm{M}_{\min }$ ). Even for hot white dwarfs however, this minimum mass increases to $0.10 \mathrm{M}_{\odot}$ for a white dwarf of $0.7 \mathrm{M}_{\odot}$ (Fig. 19), and for cooler white dwarfs with initial luminosities at the onset of accretion near $1 \%$ solar, these minimum critical masses are substantially larger (Fig. 2).

We confirm (Shen \& Bildsten 2009) that the reaction sequence ${ }^{12} \mathrm{C}(\mathrm{p}, \gamma){ }^{13} \mathrm{~N}(\alpha, \mathrm{p}){ }^{16} \mathrm{O}$ plays a critical role in accelerating the burning during the runaway, facilitating helium detonation. Including this reaction sequence had the effect of reducing the critical density required for helium detonation in a spherical model from about $1 \times 10^{6} \mathrm{~g} \mathrm{~cm}^{-3}$ to about $5 \times 10^{5} \mathrm{~g} \mathrm{~cm}^{-3}$ and this allowed lower mass helium shells to detonate. However, a study of helium detonation initiated at a point, rather than in a plane suggested that, depending upon the geometry of ignition, the required density might be $1 \times 10^{6} \mathrm{~g} \mathrm{~cm}^{-3}$ even when this reaction sequence is included $(\S 3.2)$.

At the lower densities in the smallest helium shells, helium detonation often produced intermediate mass elements, especially ${ }^{40} \mathrm{Ca}$, and not ${ }^{56} \mathrm{Ni}$. For those models where the COcore detonated, this resulted in models whose spectra and light curves more closely resembled common SN Ia (Sim et al. 2010). We also found carbon mass fractions from pre-explosive burning over $10 \%$ in the region where the detonation occurs.

Explosions with helium density so low that the nuclear time scale never becomes shorter than the convective time scale (Fig. 2) lead to helium novae, not supernovae. The subsequent evolution of these systems after ignition and their final nucleosynthesis was not followed in detail, but their composition will be rich in calcium and other lighter elements and, of course, devoid of hydrogen. Given the large nuclear energy content of the helium fuel, $\sim 10^{50} \mathrm{erg}$, these novae could, in principle, shine at the Eddington luminosity for a very long time. However, a more likely outcome is the ejection of the accreted shell over a shorter time with only partial burning of the helium. This sets the stage for recurrence.

The outcome of the other models depended critically upon how multi-dimensional aspects of the problem were approximated ( $(5)$, especially how convection was treated the last moments of the runaway $(\S$ [3.1) and the location of and temperature gradients in the vicinity of the ignition point $(\mathrm{s})(\S 3.2)$. Here, these conditions were determined by the maximum power allowed to develop in the convection zone before convection was turned off. Arguments are given ( $\S$ 3.1) that this critical luminosity lies between approximately $10^{46}$ and $10^{47}$, corresponding to a temperature at the base in the range $2.2-3.7 \times 10^{8} \mathrm{~K}$ (Fig. 2 , Table 3, $\S$ 3.1). For these conditions the nuclear burning time scale becomes shorter than 
the convective transport time scale. Once convection is halted, the temperature gradient becomes increasingly superadiabatic and the burning, localized to a small fraction of the pressure scale height. Depending upon the temperature gradient when convection freezes out, the helium may burn as a deflagration or a detonation.

Lacking clear indication of the correct choice, we explored both possibilities, though most were of the helium detonation variety. In all those models where helium detonated, the carbon core also detonated, though very fine zoning, less than $50 \mathrm{~km}$, was sometimes required to make this happen in the models with low mass helium shells. By imposing spherical or cylindrical symmetry on the ignition, helium detonation will always lead to the detonation of the carbon-oxygen white dwarf in a calculation with sufficient resolution and low numerical damping. However, we also argued that the ignition may be multi-point, asymmetric and asynchronous ( $(5.1)$. Earlier exploration of this sort of ignition in 3D models suggests that $\mathrm{CO}$ detonation by compression is not robust when cylindrical symmetry is lost (García-Senz et al. 1999). The degree of asymmetry required to cause defocusing may not be large, perhaps no more than a few hundred $\mathrm{km}(\S 5.1)$.

A substantial fraction of our models, those labeled with an "a" in Table 1 and Table 2, ignited their CO-cores more directly by a helium detonation that crossed smoothly into the carbon. However, the assumed geometry was again critical. We confirm previous studies showing that helium detonation ignited at the helium-CO interface does not yield COdetonation. Some altitude is required. Based upon calculations of critical masses of carbon and oxygen by Röpke (2007) and prior studies by García-Senz et al. (1999), we estimate that "edge-lit" carbon detonation may only happen for densities at the interface above about $3 \times 10^{6} \mathrm{~g} \mathrm{~cm}^{-3}$. At lower densities the radius of carbon required to sustain a detonation exceeds $100 \mathrm{~km}$, which is a rough upper bound on the altitude of the helium detonation. By this criterion, many of the models considered here would not detonate their carbon at the edge as indicated. Compressional ignition at the center would still occur for these onedimensional models though, and this constraint is conservative. Helium detonations are stronger than carbon detonations and are already well formed before they reach the carbon. Three-dimensional simulations of this problem are feasible and should be carried out.

Nevertheless, we focused on models that detonated their CO cores because they are the only kind of sub-Chandrasekhar mass model capable of producing a supernova resembling common Type Ia events. Most of the models in this category had helium shell masses $\sim 0.1$ $\mathrm{M}_{\odot}$ and made substantial ${ }^{56} \mathrm{Ni}$ in those shells, which they ejected with high velocity. Generally, the spectrum of these models did not resemble those of ordinary Type Ia supernovae. In some models, however, especially the hotter and heavier white dwarfs, the helium shell mass was smaller (Fig. 19). However, while sub-Chandrasekhar mass models may constitute some 
important fraction of common SN Ia, there are a number of potential objections to them as a general solution to the "SN Ia problem". Some can be addressed with further computation and may be resolved shortly. Others might be addressed with further observation.

1) The models that resemble closely SN Ia, for example 9HC, 10HC (Fig. 15), and 11HD, occupy a relatively narrow niche of parameter space (Fig. 19). For low luminosity white dwarfs, none are found, and for high luminosity white dwarfs, the necessary range of accretion rates is quite narrow. Why, for example, does a $1.0 \mathrm{M}_{\odot}$ white dwarf have $\mathrm{L}=\mathrm{L}_{\odot}$ and not $\mathrm{L}=0.01 \mathrm{~L}_{\odot}$ and $\dot{\mathrm{M}}=4 \times 10^{-8} \mathrm{M}_{\odot} \mathrm{y}^{-1}$ and not 3 or $5 \times 10^{-8} \mathrm{M}_{\odot} \mathrm{y}^{-1}$ ?

One possibility is that the explosion occurs in a binary system with a gradually declining accretion rate so that repeated nova-like outbursts precede a single helium detonation (Bildsten et al. 2007). The nova-like outbursts could have left the outer layers of the white dwarf in a heated state and our "hot white dwarf models" might then be appropriate. The last flash would have a helium shell mass just over the minimum value for helium detonation and could trigger a $\mathrm{CO}$ detonation that destroyed the system. But then the accretion rate must always decline just enough to produce a detonation with a minimum mass and not so much as to give a thick helium shell. Also, the mass of the white dwarf must increase during the presupernova evolution to $1.1 \mathrm{M}_{\odot}$ to explain typical SN Ia luminosities. Nova-like outbursts at the high accretion rate will actually shrink the white dwarf mass. Perhaps this can all be made to work, but is it so much harder to grow the mass to $1.38 \mathrm{M}_{\odot}$ (and make a Chandrasekhar-mass explosion), rather than $1.1 \mathrm{M}_{\odot}$ ?

2) Despite numerous papers on the subject, neither the detonation of the helium nor of the carbon has been conclusively demonstrated, especially for densities less than $10^{6}$ $\mathrm{g} \mathrm{cm}^{-3}$ (§ 3.2). Helium detonation can be averted if shallow temperature gradients are not maintained by efficient convection at late times. Even if the helium detonates, carbon detonation is not assured for the general case of asymmetric, asynchronous, multi-point ignition ( $(5)$. The existence of supernovae like SN 2010X Kasliwal et al. (2010)) is also suggestive that, at least occasionally, helium detonation or deflagration is realized without detonating the $\mathrm{CO}$ core.

3) Even the models that closely resemble SN Ia differ in subtle ways. The spectral features due to intermediate mass elements, while present in the models, are not as deep as seen in many normal SNe Ia. In addition, the minimum velocity of IME in the models is typically no lower than $11,000 \mathrm{~km} \mathrm{~s}^{-1}$, whereas in observed SNe Ia the IMEs are generally observed to extend to lower velocities, $v \approx 9,000 \mathrm{~km} \mathrm{~s}^{-1}$. We caution, however, that ours are 1-D models and both the nucleosynthesis and degree of radial mixing of intermediate mass elements may differ in multi-D models. 
4) Past models may have erred in taking too low a density for the helium shell when it detonated. This density came from assuming a) that the criterion for transitioning to a hydrodynamical simulation was the nuclear time scale equal the sonic time scale when it should be the nuclear time scale equals the convective transport time scale. Also the conditions at the runaway point were adopted for the whole helium shell, not just the small locus of ignition. Many of our models also ignite well above the CO core - helium interface so there is denser helium beneath.

If the CO-core does not detonate all the time, there could be a diverse set of fainter transients awaiting discovery. These could range in peak brightness all the way from bright radioactive powered novae $\left(\sim 10^{39} \mathrm{erg} \mathrm{s}^{-1}\right)$ to subluminous Type Ia supernovae (Fig. 6). The faintest events would be powered by just a trace of ${ }^{48} \mathrm{Cr}$ (e.g., Fig. 18 and Models 7B1, 9A1, 10HC1, and 10HCD1; see also Bildsten et al. 2007). Each leaves behind a hot white dwarf that might potentially be detectable for thousands of years (Woosley et al. 1986). For lower accretion rates and cooler white dwarfs, larger masses of ${ }^{56} \mathrm{Ni}$ and smaller masses of ${ }^{48} \mathrm{Cr}$ and ${ }^{52} \mathrm{Fe}$ are produced resulting in a brighter supernova - though still much fainter than an ordinary SN Ia. If these transients exist, they await discovery.

A novel outcome explored here is helium deflagration. If the temperature gradient at the base of the helium shell is too steep at the time of the final runaway to produce a supersonic phase velocity for the burning rate and form a detonation, the burning will continue to steepen that gradient until a strong density inversion develops. The rise of buoyant burning material leads to mixing that accelerates the burning rate. In about $10 \mathrm{~s}$, the burning could sweep around the star, but no supersonic speeds are developed anywhere. We modeled this sort of explosion ( $\S 3.3$ using a Sharp-Wheeler model for the advancement of the RayleighTaylor instability. This is a gross approximation, and the value used for the uncertain constant "A" in eq. (9), which determines the degree of burning, was on the high side of its expected range. Any realistic model will have to be computed in multi-dimensions (though see $\S 5.3$. However, some generic features may survive. First, far less helium burns than in a detonation so the supernova is less energetic; the speeds are much slower. Second, for a given density, burning in a deflagration occurs at a lower temperature than in a detonation. For the two models studied, substantial amounts of ${ }^{44} \mathrm{Ti}$ and ${ }^{48} \mathrm{Cr}$ were produced and little ${ }^{56} \mathrm{Ni}$. These transients would be quite faint. Detonation in the same models gave large energies and large ${ }^{56} \mathrm{Ni}$ mass fractions.

As noted in previous studies, the nucleosynthesis from the sub-Chandrasekhar mass models is acceptable, perhaps even superior to that reported for standard Chandrasekhar mass models. Large overproductions of ${ }^{58} \mathrm{Ni}$ are avoided in the sub-Chandrasekhar mass models, and important contributions to ${ }^{44} \mathrm{Ca},{ }^{47} \mathrm{Ti},{ }^{63,65} \mathrm{Cu}$ and ${ }^{64} \mathrm{Zn}$ are made $(\S$ 6; , Tables 
5 - 10). Especially important is the species ${ }^{44} \mathrm{Ca}$, made as ${ }^{44} \mathrm{Ti}$. The ratio of ${ }^{44} \mathrm{Ca} /{ }^{56} \mathrm{Fe}$ is subsolar in models where the entire star detonates and the CO-core exceeds one solar mass. But lighter models, especially the helium deflagrations can produce large ratios of ${ }^{44} \mathrm{Ca}$ to iron. Since ${ }^{44} \mathrm{Ca}$ is inadequately produced in both massive stars and Chandrasekhar-mass SN Ia, the present abundance of ${ }^{44} \mathrm{Ca}$ in the sun thus a strong argument that sub-Chandrasekhar mass explosions have occurred. It further suggests an important component in which either the CO-core is lighter than $1 \mathrm{M}_{\odot}$ or does not detonate. These would not be ordinary SN Ia. A vexing uncertainty in these models, however, are the poorly determined reaction rates for unstable nuclei heavier than calcium with $\mathrm{Z} \approx \mathrm{N}$. While we did not carry out a sensitivity study, we expect factor of two or more variations in key abundances could occur as a consequence of uncertain rates (e.g. Hoffman et al. 2010). With present choices for these rates the large production of ${ }^{48} \mathrm{Ti}$ and ${ }^{61} \mathrm{Ni}$ (as ${ }^{48} \mathrm{Cr}$ and ${ }^{61} \mathrm{Zn}$ ) often preclude the production of anything else.

We explored the spectra and light curves of a representative subset of our models and found, in agreement with Sim et al. (2010), that a wide range of luminosities are possible for the SN Ia resulting from sub-Chandrasekhar mass explosions that detonate their CO cores, even very brilliant ones if the requisite accretion rate and CO-dwarf mass are realized. These luminosities obey, roughly, a width luminosity relation, however the slope and normalization do not agree well with recent observations. In general, the models decline more rapidly in the B-band than do the observations. We differ fundamentally with Sim et al. (2010) and Kromer et al. (2010) regarding the nucleosynthesis expected from helium detonation in these models $(\S 6.2)$.

The observational counterparts of the high helium shell mass, double-detonation models remain unclear. These did not closely resemble those of common SN Ia. In all cases, the presence of the outer shell led to a peculiar, fairly featureless spectrum around maximum light. This problem is due in part to the opacity of iron-group element in the shell, as has been emphasized in previous studies. We find in addition that the dynamical effects of the shell are important - the mass of the shell decelerates the detonated core, thereby restricting the velocity spread of the IME ejected in the CO detonation to a narrow shell $\sim 11,000-13,000 \mathrm{~km} \mathrm{~s}^{-1}$. This reduces the strength and blueshift of the IME absorption features and is largely responsible for the poor fit to observed SNe Ia. In order to reproduce the spectra of normal SNe Ia, the mass in the helium shell should be small, $\lesssim 0.05 \mathrm{M}_{\odot}$ and should be relatively free of radioactive isotopes. It is possible that some explosions with larger helium shells could be be associated with the spectroscopically peculiar class of SN 1999aa or SN 19991T-like events. Or perhaps such low accretion rates are not frequently sustained long enough to cause an explosion in nature. 
Models in which only the shell explodes produce relatively dim and fast transients. Within this class, we found a wide range of peak brightness (peak B-band magnitudes ranging from -13.5 to -18.5) and diverse spectroscopic properties. In the models which assumed an initially "cold" white dwarf, the helium shell detonations generally produced transients powered by ${ }^{56} \mathrm{Ni}$ decay. The spectra were dominated by titanium and iron group lines and lacked IME features, similar to the ".Ia" explosions studied by Shen et al. (2010). The "hot" white dwarf models, on the other hand, generally produced lower abundances of ${ }^{56} \mathrm{Ni}$ and were powered mainly by ${ }^{48} \mathrm{Cr}$. Their spectra showed strong IME features, similar to low-luminosity SNe Ia like SN 1991bg. The models in which the helium shell was assumed to explode by deflagration, rather than detonation, had distinct observational properties altogether. The velocities of line absorptions in these models were extremely low $\left(\sim 4000 \mathrm{~km} \mathrm{~s}^{-1}\right)$. Calcium lines were strong, and some of the features were due to unusual species such as Sc II. Spectral lines from (radioactive) titanium and vanadium might also be searched for. Only two such shell deflagrations were modeled here, each with peak B-band magnitudes around -15 , but in principle a wider range of luminosities and durations are possible, depending on the mass of the shell at the time of explosion. We suggest that the scenario may be relevant for explaining the class of dim transients showing low velocities in their spectra (e.g., SN 2008ha Foley et al. 2009).

Fortunately, it is feasible to do all these problems in three dimensions. The convective ignition can be studied using a low Mach number code (e.g. Nonaka et al. 2010), and several groups are developing the capabilities to study multi-dimensional deflagrations and detonations. Three dimensional light curves and spectra can also be computed (Kasen et al. 2007). Given the forthcoming all sky surveys that will discover many new optical transients, and the probability that phenomena similar to those explored here should exist at some level, we expect this to be a fertile field for further exploration.

This research has been supported by the DOE SciDAC Program under contract DEFC02-06ER41438; the National Science Foundation (AST 0909129) and the NASA Theory Program (NNX09AK36G). We acknowledge useful discussions with Ron Taam on the treatment of accretion in Lagrangian codes, and with Fritz Röpke, Stuart Sim, and Michael Fink, helping to elucidate the differences between our models and theirs. Rob Hoffman and Alex Heger helped develop the reaction network and Kepler code. Discussions with Gary Glatzmaier and Mike Zingale helped to elucidate the nature of the baroclinic instability. Correspondence with Ken Shen and Lars Bildsten helped to explore important aspects of accretion, helium detonation, and of their published works. The anonymous referee was extraordinarily helpful in helping us to clarify and, in some cases, substantially revise, our major conclusions. 


\section{REFERENCES}

Arnett, D. 1997, NATO ASIC Proc. 486: Thermonuclear Supernovae, 405

Ashok, N. M., \& Banerjee, D. P. K. 2003, A\&A, 409, 1007

Aspden, A. J., Bell, J. B., Day, M. S., Woosley, S. E., \& Zingale, M. 2008, ApJ, 689, 1173

Benz, W. 1997, NATO ASIC Proc. 486: Thermonuclear Supernovae, 457

Bhattacharyya, S., \& Strohmayer, T. E. 2006, ApJ, 642, L161

Bildsten, L., Shen, K. J., Weinberg, N. N., \& Nelemans, G. 2007, ApJ, 662, L95

Blinnikov, S. I., \& Khokhlov, A. M. 1987, Pis ma Astronomicheskii Zhurnal, 13, 868

Buchmann, L. 1996, ApJ, 468, L127

Casanova, J., José, J., García-Berro, E., Calder, A., \& Shore, S. N. 2010, A\&A, 513, L5

Caughlan, G. R., \& Fowler, W. A. 1988, Atomic Data and Nuclear Data Tables, 40, 283

Damköhler, G. 1940, Z. Elektrchem, 46, 601

Dursi, L. J., \& Timmes, F. X. 2006, ApJ, 641, 1071

Filippenko, A. V. et al. 1992, ApJ, 384, L15

Fink, M., Hillebrandt, W., Röpke, F. K. 2007, A\&A, 476, 1133

Fink, M., Röpke, F. K., Hillebrandt, W., Seitenzahl, I. R., Sim, S. A., \& Kromer, M. 2010, arXiv:1002.2173

Folatelli, G. et al. 2010, AJ, 139, 120

Foley, R. J. et al. 2009, AJ, 138, 376

Fryxell, B. A., \& Woosley, S. E. 1982, ApJ, 261, 332

García-Senz, D., Bravo, E., \& Woosley, S. E. 1999, A\&A, 349, 177

Glasner, S. A., Livne, E., \& Truran, J. W. 2007, ApJ, 665, 1321

Glimm, J., \& Li, X. L. 1988, Physics of Fluids, 31, 2077

Hashimoto, M.-A., Nomoto, K.-I., Arai, K., \& Kaminisi, K. 1986, ApJ, 307, 687 
Hoeflich, P., \& Khokhlov, A. 1996, ApJ, 457, 500

Hoffman, R. D., et al. 2010, ApJ, 715, 1383

Iben, I., Jr., \& Tutukov, A. V. 1991, ApJ, 370, 615

Iwamoto, K., Brachwitz, F., Nomoto, K., Kishimoto, N., Umeda, H., Hix, W. R., \& Thielemann, F.-K. 1999, ApJS, 125, 439

Kasen, D. 2006, ApJ, 649, 939

Kasen, D., Röpke, F. K., \& Woosley, S. E. 2009, Nature, 460, 869

Kasen, D., Thomas, R. C., \& Nugent, P. 2006, ApJ, 651, 366

Kasen, D., Woosley, S., Nugent, P., Röpke, F. 2007, Journal of Physics Conference Series, 78,012037

Kasliwal, M. M., et al. 2010, ApJ, 723, L98

Kato, M., \& Hachisu, I. 2003, ApJ, 598, L107

Kromer, M., Sim, S. A., Fink, M., Röpke, F. K., Seitenzahl, I. R., \& Hillebrandt, W. 2010, ApJ, 719, 1067

Landau, L., \& Lifshitz, F. M. 1959, Course in Theoretical Physics, Vol. 6. Fluid Mechanics (Oxford:Pergammon)

Li, W., Filippenko, A. V., Treffers, R. R., Riess, A. G., Hu, J., \& Qiu, Y. 2001, ApJ, 546, 734

Livne, E. 1990, ApJ, 354, L53

Livne, E., \& Glasner, A. S. 1990, ApJ, 361, 244

Livne, E., \& Glasner, A. S. 1991, ApJ, 370, 272

Livne, E., \& Arnett, D. 1995, ApJ, 452, 62

Livne, E. 1997, NATO ASIC Proc. 486: Thermonuclear Supernovae, 425

Lodders, K. 2003, ApJ, 591, 1220

Niemeyer, J. C., \& Woosley, S. E. 1997, ApJ, 475, 740

Nomoto, K. 1980, Space Science Reviews, 27, 563 
Nomoto, K. 1982a, ApJ, 253, 798

Nomoto, K. 1982b, ApJ, 257, 780

Nomoto, K., Thielemann, F.-K., \& Miyaji, S. 1985, A\&A, 149, 239

Nonaka, A., Almgren, A. S., Bell, J. B., Lijewski, M. J., Malone, C. M., \& Zingale, M. 2010, ApJS, 188, 358

Nugent, P., Baron, E., Branch, D., Fisher, A., \& Hauschildt, P. H. 1997, ApJ, 485, 812

Perets, H. B. et al. 2010, Nature, 465, 322

Pinto, P. A., \& Eastman, R. G. 2000, ApJ, 530, 757

Plewa, T., Calder, A. C., \& Lamb, D. Q. 2004, ApJ, 612, L37

Poznanski, D. et al. 2010, Science, 327, 58

Renedo, I., Althaus, L. G., Miller Bertolami, M. M., Romero, A. D., Córsico, A. H., Rohrmann, R. D., \& García-Berro, E. 2010, ApJ, 717, 183

Rauscher, T., Heger, A., Hoffman, R. D., \& Woosley, S. E. 2002, ApJ, 576, 323

Röpke, F. K. 2007, ApJ, 668, 1103

Röpke, F. K., Woosley, S. E., \& Hillebrandt, W. 2007, ApJ, 660, 1344

Shen, K. J., \& Bildsten, L. 2009, ApJ, 699, 1365

Shen, K. J., Kasen, D., Weinberg, N. N., Bildsten, L., \& Scannapieco, E. 2010, ApJ, 715, 767

Sim, S. A., Röpke, F. K., Hillebrandt, W., Kromer, M., Pakmor, R., Fink, M., Ruiter, A. J., \& Seitenzahl, I. R. 2010, ApJ, 714, L52

Spitkovsky, A., Levin, Y., \& Ushomirsky, G. 2002, ApJ, 566, 1018

Stanishev, V. et al. 2007, A\&A, 469, 645

Taam, R. E. 1980a, ApJ, 242, 749

Taam, R. E. 1980b, ApJ, 237, 142

Timmes, F. X., \& Woosley, S. E. 1992, ApJ, 396, 649 
Timmes, F. X., Woosley, S. E., Hartmann, D. H., \& Hoffman, R. D. 1996, ApJ, 464, 332

Timmes, F. X., \& Swesty, F. D. 2000, ApJS, 126, 501

Timmes, F. X., \& Niemeyer, J. C. 2000, ApJ, 537, 993

Weaver, T. A., Zimmerman, G. B., \& Woosley 1978, ApJ, 225, 1021 York Academy Sciences Annals, 470, 267

Woosley, S. E., Weaver, T. A., \& Taam, R. E. 1980, Texas Workshop on Type I Supernovae, 96

Woosley, S. E., \& Wallace, R. K. 1982, ApJ, 258, 716

Woosley, S. E., Taam, R. E., \& Weaver, T. A. 1986, ApJ, 301, 601

Woosley, S. E., \& Weaver, T. A. 1988, Phys. Rep., 163, 79

Woosley, S. E. 1990, in Supernovae, ed. A Petschek, Springer Verlag, p. 182

Woosley, S. E., \& Weaver, T. A. 1994, ApJ, 423, 371

Woosley, S. E., Heger, A., \& Weaver, T. A. 2002, Reviews of Modern Physics, 74, 1015

Woosley, S. E., Wunsch, S., \& Kuhlen, M. 2004, ApJ, 607, 921

Woosley, S. E., \& Heger, A. 2007, Phys. Rep., 442, 269

Woosley, S. E., Kerstein, A. R., Sankaran, V., Aspden, A. J., Röpke, F. K. 2009, ApJ, 704, 255

Yoon, S.-C., \& Langer, N. 2004, A\&A, 419, 645

Yousaf, M. 1986, Physics of Fluids, 29, 680

Zel'Dovich, Ya. B., \& Raizer, Yu. P. 1966, Physics of Shock Waves and High-Temperature Hydrodynamic Phenomena, [Dover, 2002], Section XII, p. 794.

Zel'Dovich, Ya. B., Librovich, V. B., Makhviladze, G. M., \& Sivashinskil, G. I. 1970, Journal of Applied Mechanics and Technical Physics, 11, 264

Zingale, M., Woosley, S. E., Rendleman, C. A., Day, M. S., \& Bell, J. B. 2005, ApJ, 632, 1021

Zingale, M., Almgren, A. S., Bell, J. B., Nonaka, A., \& Woosley, S. E. 2009, ApJ, 704, 196 
This preprint was prepared with the AAS LATEX macros v5.0. 

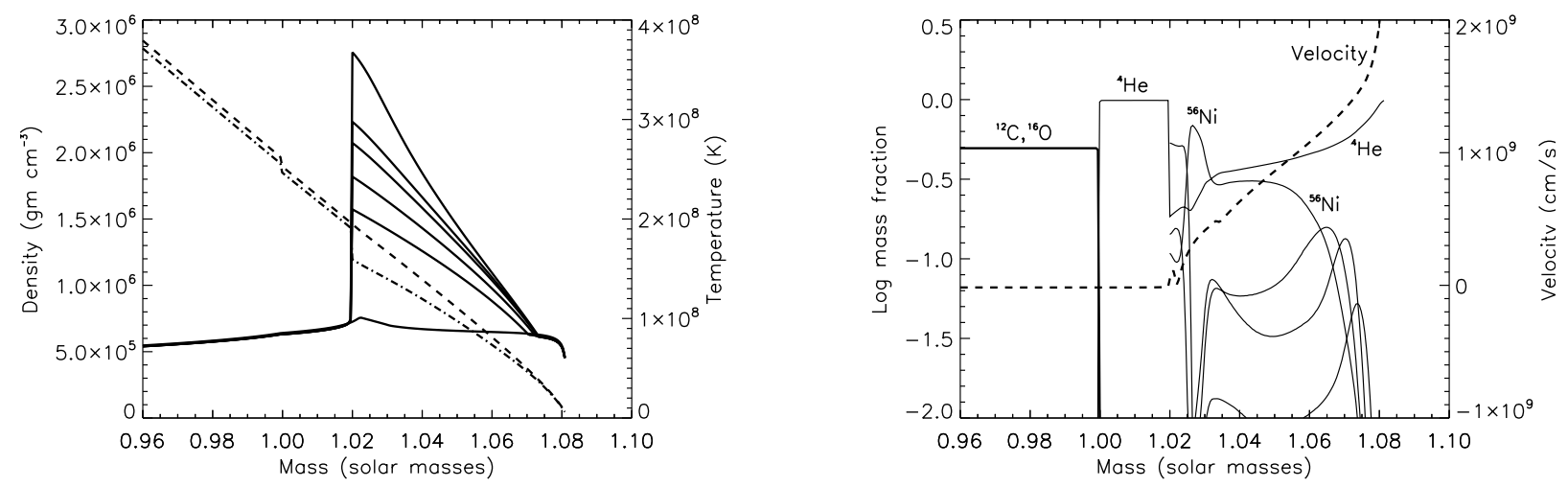

Fig. 1. - The left frame shows the history of the temperature and density in Model 10B as the helium runaway develops. Solid lines, from bottom to top, give the temperature profile a) when nuclear burning first becomes adequate to cause convection in the accreted layer; b) the maximum convective luminosity in the shell equals $10^{45} \mathrm{erg} \mathrm{s}^{-1}$; c) $10^{46} \mathrm{erg} \mathrm{s}^{-1}$; d) $5 \times 10^{46} \mathrm{erg} \mathrm{s}^{-1}$; e) $10^{47} \mathrm{erg} \mathrm{s}^{-1}$, and f) $5 \times 10^{47} \mathrm{erg} \mathrm{s}^{-1}$. The dashed lines show the density at first ignition (point a) and at $\mathrm{L}=5 \times 10^{47} \mathrm{erg} \mathrm{s}^{-1}$ (point f). When convection is left on until point $\mathrm{f}$ and then turned off, an outwards propagating detonation wave develops quickly, but no inwards detonation occurs, despite the substantial separation between the ignition point and helium shell - CO-core interface at $1.0 \mathrm{M}_{\odot}$. The right hand frame shows some of the abundant isotopes after the helium detonation is over and their velocities. The unlabeled solid curves are from top to bottom, ${ }^{52} \mathrm{Fe},{ }^{48} \mathrm{Cr}$, and ${ }^{44} \mathrm{Ti}$. In this Model $10 \mathrm{~B} 1$ there was no detonation induced in the CO-white dwarf. 


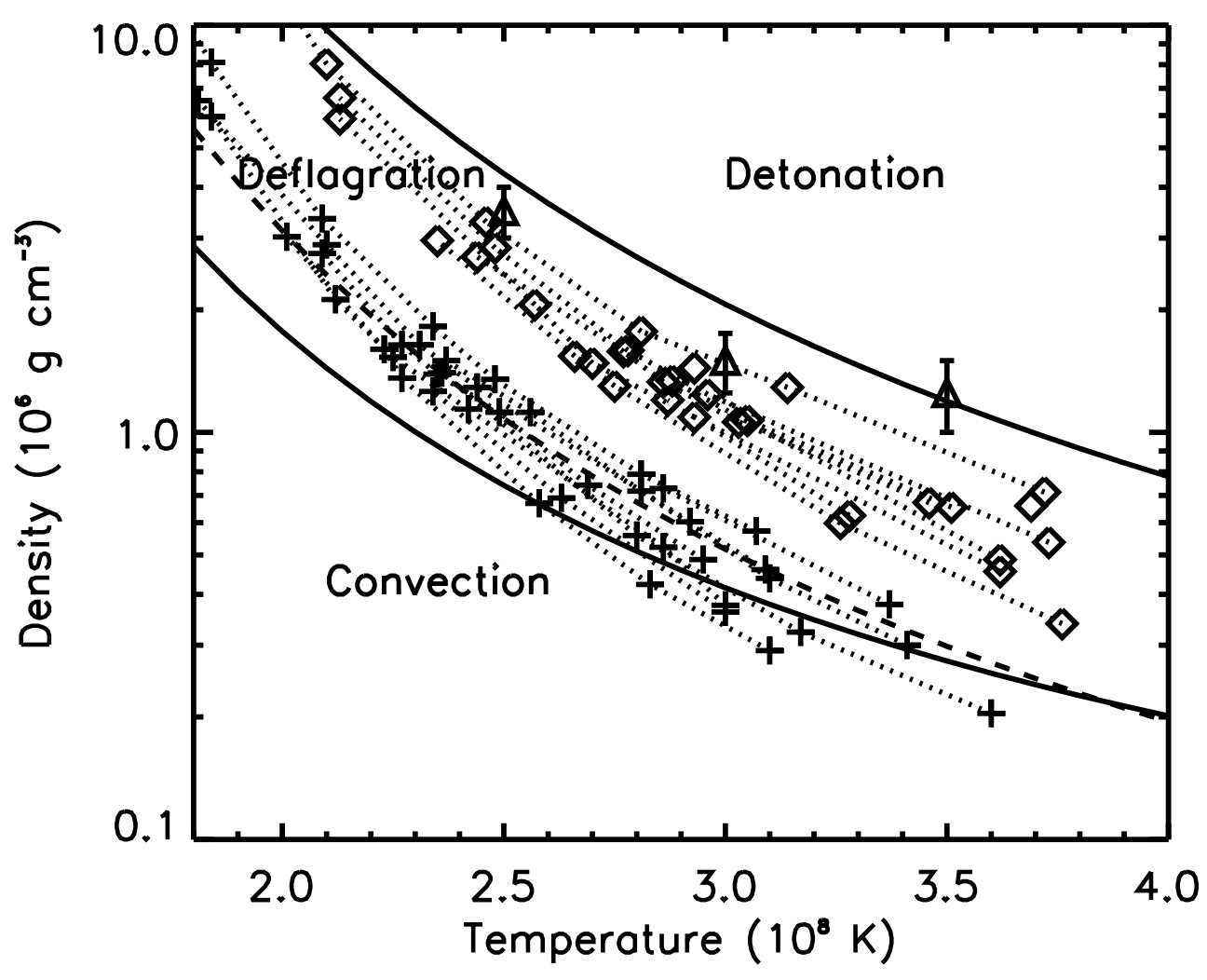

Fig. 2. - Temperatures and densities at the base of the helium burning shell when the peak luminosity in that shell reaches $10^{46} \mathrm{erg} \mathrm{s}^{-1}$ ("+" symbols) and $10^{47} \mathrm{erg} \mathrm{s}^{-1}$ (diamond symbols). Dotted lines connect the ignition conditions in Table 3 for series of models based upon the same CO white dwarf mass and temperature, but with different accretion rates. The upper solid line is the analytic condition for detonation (eq. (7)) and the bottom solid line is the condition $\tau_{\text {run }}=2 \mathrm{~s}$, a nominal condition for the first break down of convection. In between these two lines a deflagration can occur, a subsonic runaway that is transported neither by convection or detonation. In practice, all of the $10^{47} \mathrm{erg} \mathrm{s}^{-1}$ points detonated and most of the $10^{46} \mathrm{erg} \mathrm{s}^{-1}$ points, showing that planar detonation is easier than suggested by eq. (7). Dividing the top line by an arbitrary factor of 4 gives the dashed line that is more consistent with the model results. Triangles with error bars at $\mathrm{T}_{8}=2.5,3.0$, and 3.5 show the location of the detonation transition as determined by fine-zoned hydrodynamical studies where the ignition occurred at a point rather than a plane. These data points are more consistent with the solid line given by eq. (7) rather than the dashed one and suggest that a) no detonation occurs for a density less than $10^{6} \mathrm{~g} \mathrm{~cm}^{-3}$, and b) there could be an appreciable range of models that will deflagrate rather than detonate. 

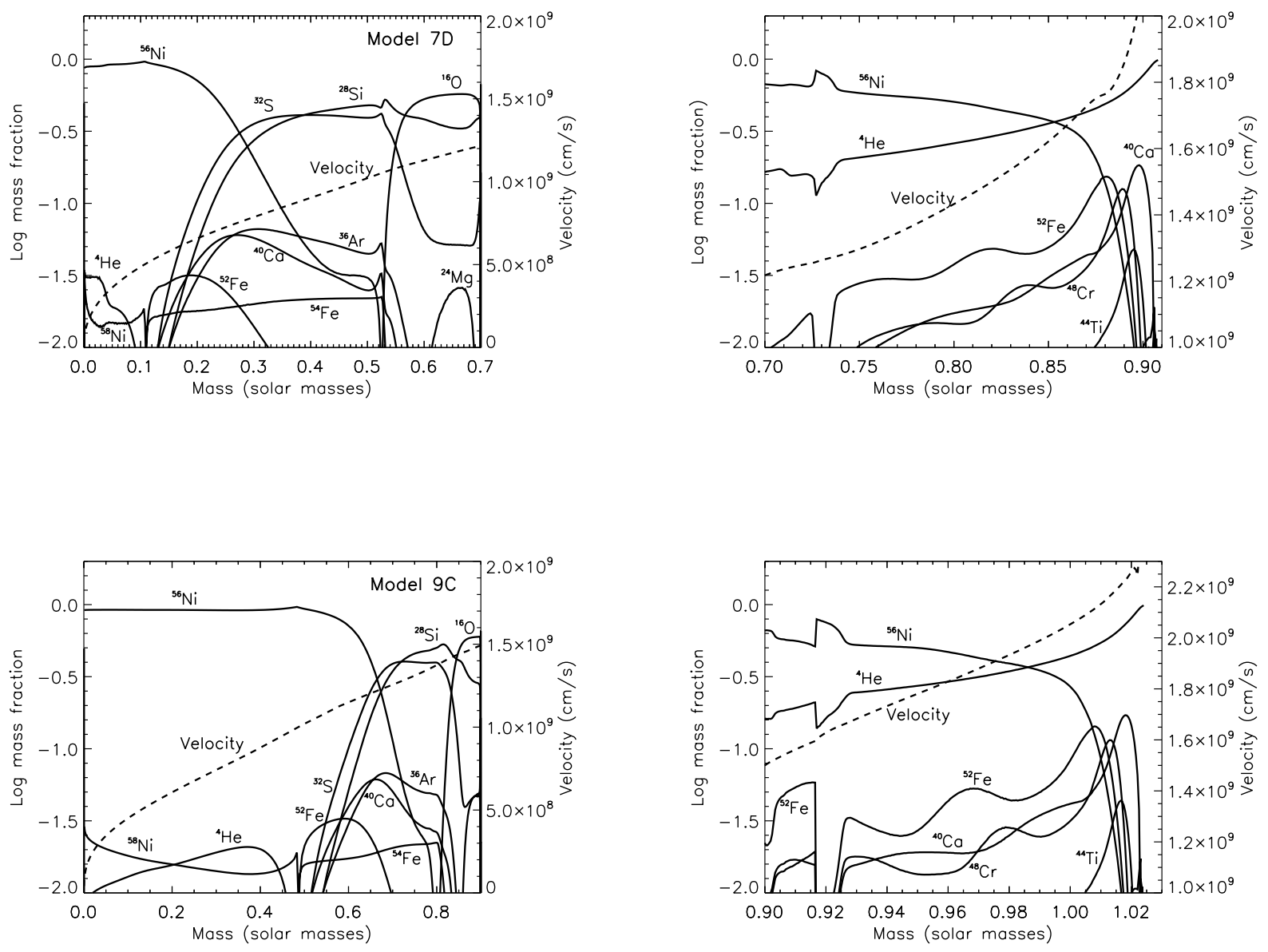

Fig. 3.- Final abundances and velocity in Models 7D and $9 \mathrm{C}$ showing a range of nickel and intermediate mass synthesis. In each case the left frame shows the log of the mass fraction for species produced within the original CO-core and the right frame shows details of the helium shell detonation. The total mass for Model $7 \mathrm{D}$ was $0.907 \mathrm{M}_{\odot}$ and ignition occurred at $0.725 \mathrm{M}_{\odot}$. Ignition resulted in both outwards and inwards moving detonations in the helium layer so that the whole star exploded. The total mass of Model 9C was $1.02 \mathrm{M}_{\odot}$ and ignition occurred at $0.918 \mathrm{M}_{\odot}$. The prompt part of the explosion consisted of an outwards moving helium detonation but compression resulted, with some delay, in a secondary, centrally ignited detonation of the CO core so that, in the end the whole star exploded. Because of its larger total mass and higher density, less intermediate mass elements are produced in Model 9C. 

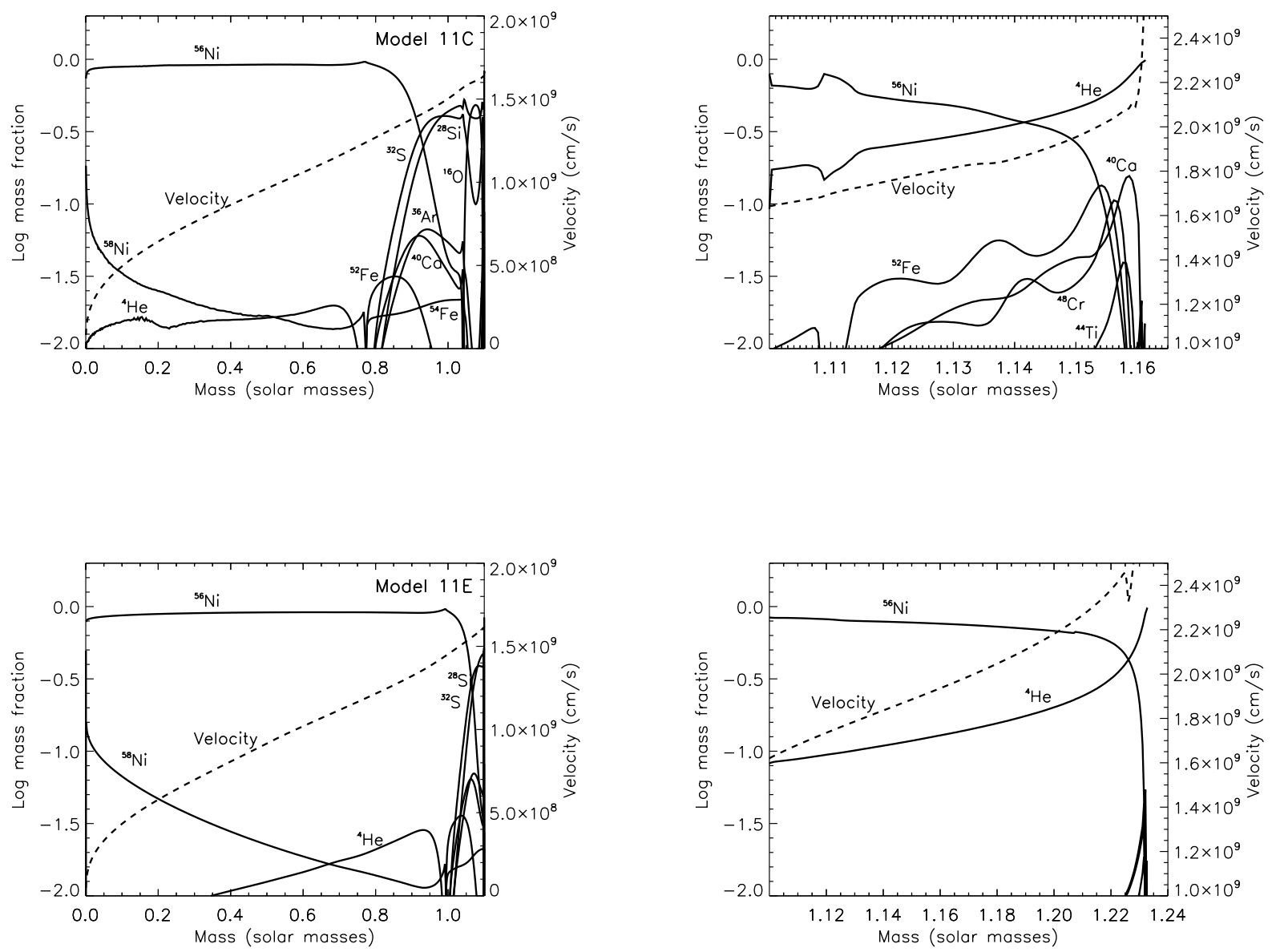

Fig. 4.- Final abundances and velocities in two models with higher mass and density. These will make bright SN Ia with weaker lines of intermediate mass elements. Model 11C had a total mass of $1.162 \mathrm{M}_{\odot}$ and ignited at $1.11 \mathrm{M}_{\odot}$. Model $11 \mathrm{E}$ had a total mass of $1.233 \mathrm{M}_{\odot}$ and ignited at the CO-He interface. Model 11C had a double detonation of the helium shell which propagated to the center of the star. Model 11E had an outwards moving helium detonation and a secondary central carbon detonation. 


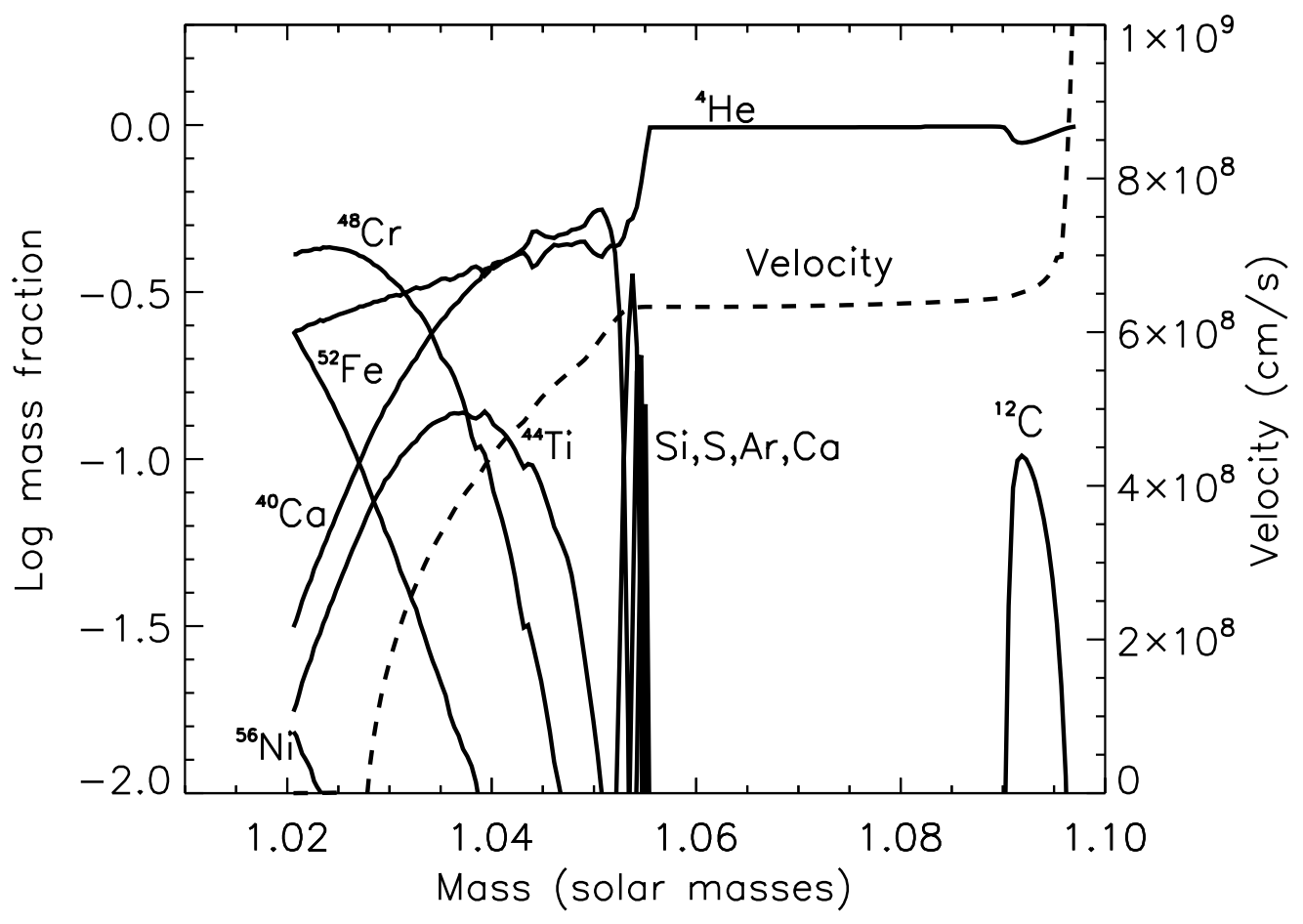

Fig. 5.- Final abundances and velocity in Model 10DEFL. Burning is at a lower temperature and in a smaller fraction of the mass than in the models that detonate. Consequently the explosion speed is lower. The near constant speed in the unburned helium layer is a consequence of the one-dimensional nature of the simulation and would be different in 2D or $3 \mathrm{D}$. 

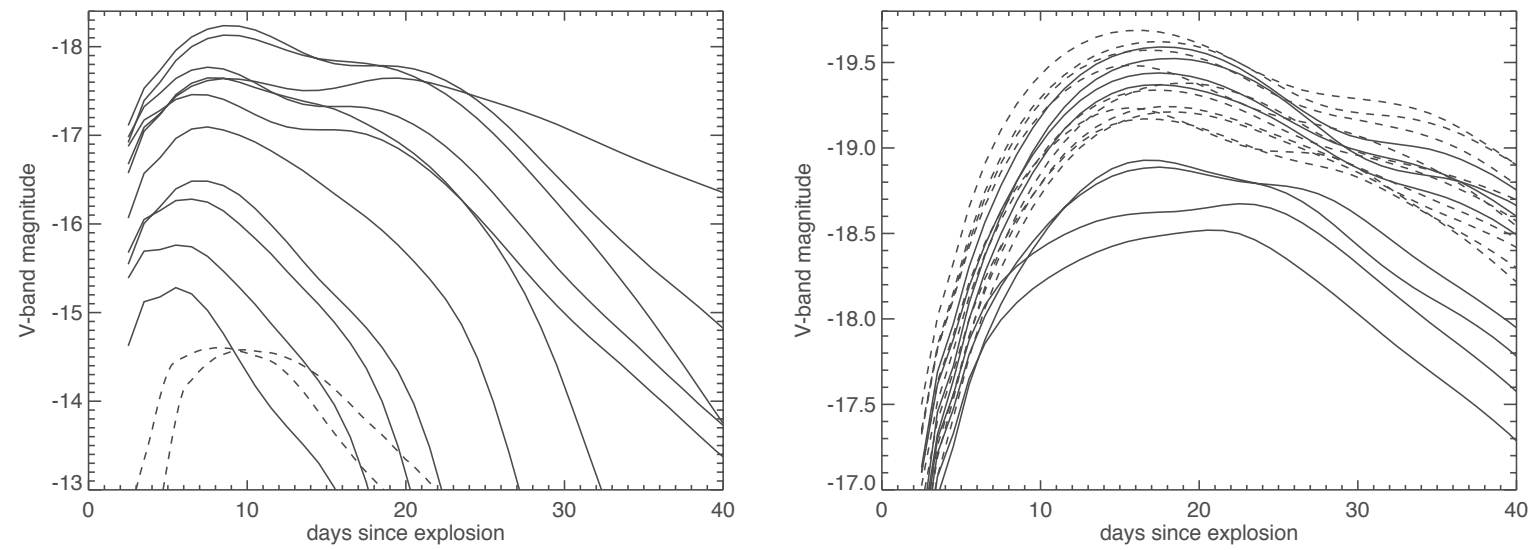

Fig. 6. - A sample of V-band light curves illustrating the diversity of observable behaviors seen in the model set. Left: Models in which only the helium shell exploded, either by detonation (case c, solid lines) or deflagration (case b, dashed lines). Right: Models in which the entire star exploded, either by an inward detonation (case a, solid lines) or by compression of the CO-core triggering a secondary detonation (case d, dashed lines). 


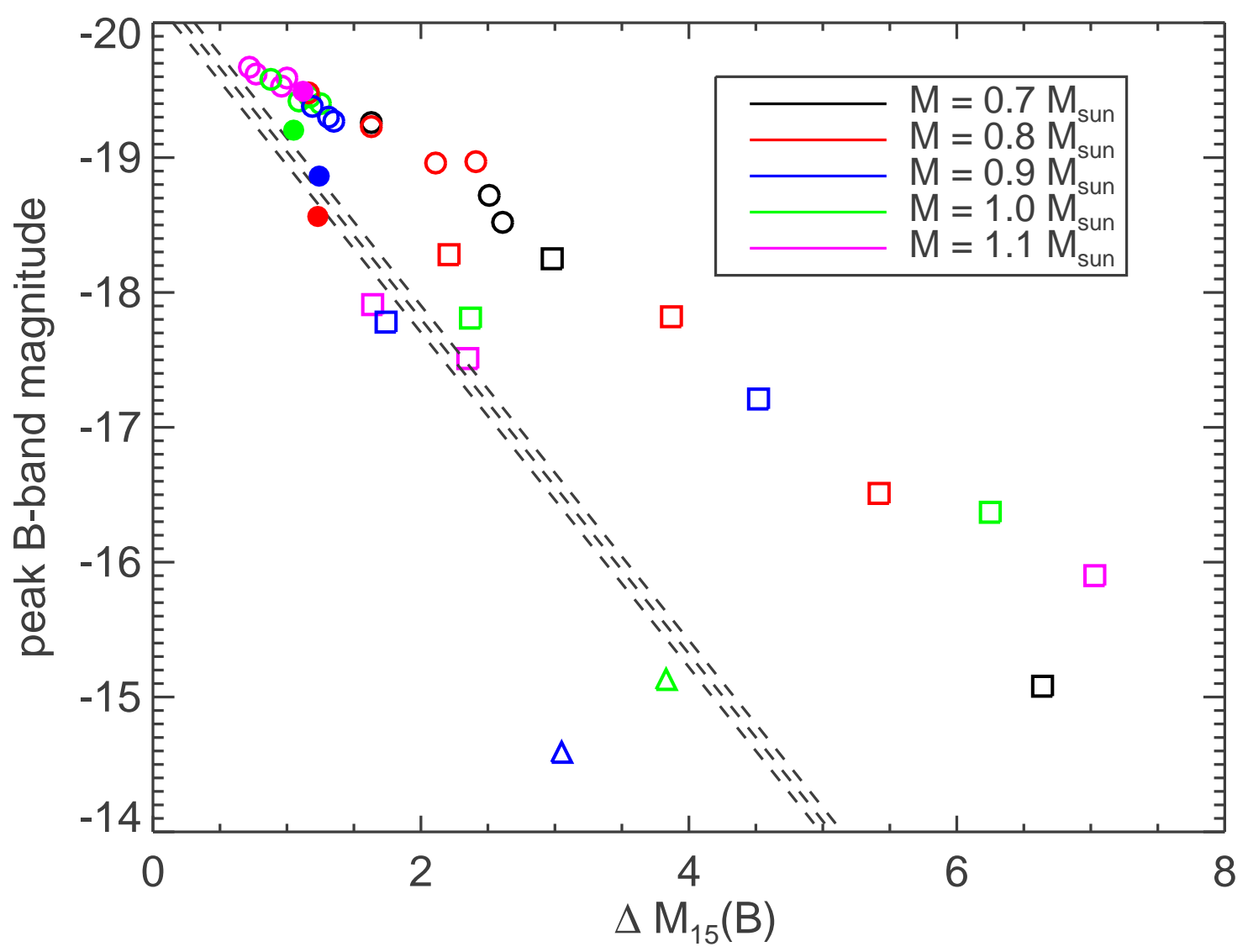

Fig. 7.- Width-luminosity relation for the models. Open symbols represent "cold" models (i.e., those where the white dwarf had an initial luminosity of $0.01 \mathrm{~L}_{\odot}$ ) while the closed circles represent four "hot" models (initial luminosity $L=1 \mathrm{~L}_{\odot}$; Models 8HBC, 9HC, 10HC, and 11HD). Squares denote models where only the helium shell detonated, triangles, models where the shell deflagrated, and circles, models where both the helium shell and CO-core exploded. Color coding indicates the initial white dwarf mass. The hatched region shows the observed width-luminosity relation of Folatelli et al. (2010): $M_{B}=-19.12+1.241\left(\Delta M_{15}(B)-1.1\right)$, with a \pm 0.1 mag spread. This linear relation has been naively extrapolated into the region to $\Delta M_{15}(B)>1.8$ where it actually no longer holds. 


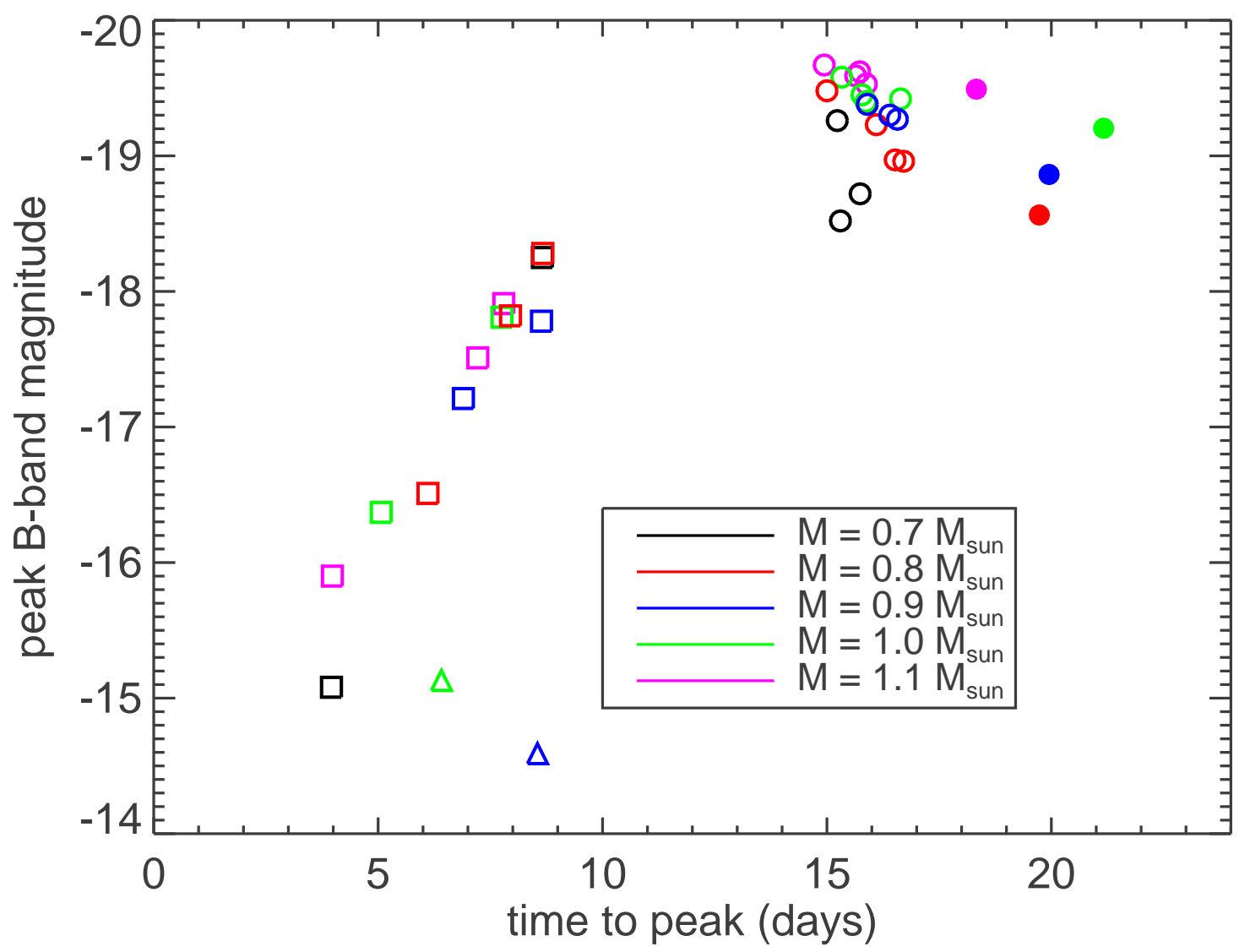

Fig. 8.- Peak B-band luminosity versus the B-band rise time for the models described in Fig. 7. Open (closed) symbols represent models with cold (hot) white dwarfs. Squares denote models where only the helium shell detonated; triangles, models where the helium shell deflagrated, and circles, models where both the shell and core exploded. Color coding indicates the initial white dwarf mass. 


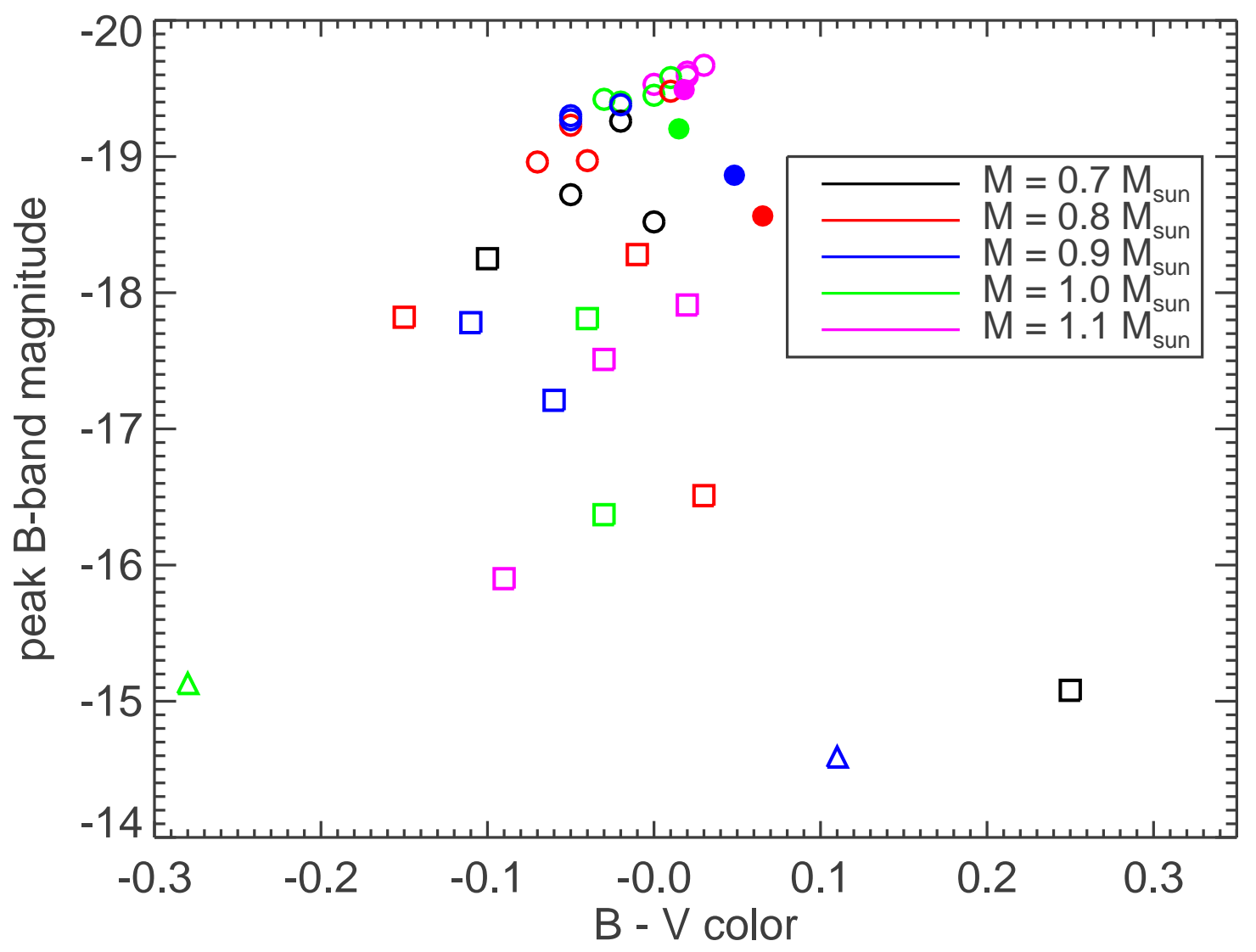

Fig. 9.- Peak B-band luminosity versus the B-V color at B-maximum for the models described in Fig. 7. Open (closed) symbols represent models with cold (hot) white dwarfs.. Squares denote models where only the helium shell detonated; triangles, models where the shell deflagrated; and circles, models where both shell and core exploded. The color coding indicates the initial white dwarf mass. 


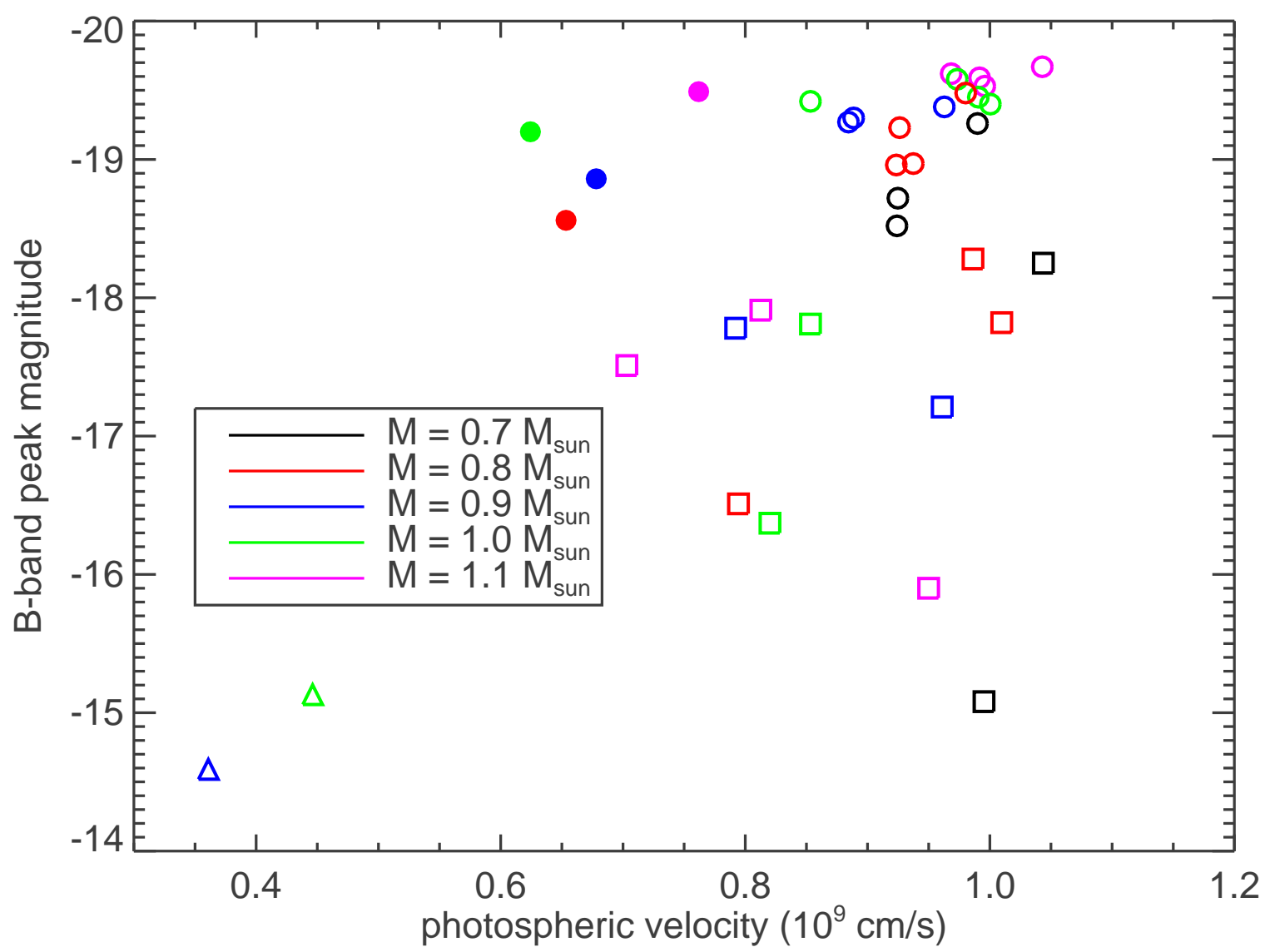

Fig. 10.- Peak B-band luminosity versus the velocity of the electron scattering photosphere at B-maximum for the models described in Fig. 7. Open (closed) symbols represent models with cold (hot) white dwarfs. Squares denote models where only the shell detonated, triangles models where the shell deflagrated, and circles models where both shell and core exploded. Color coding indicates the initial white dwarf mass. 


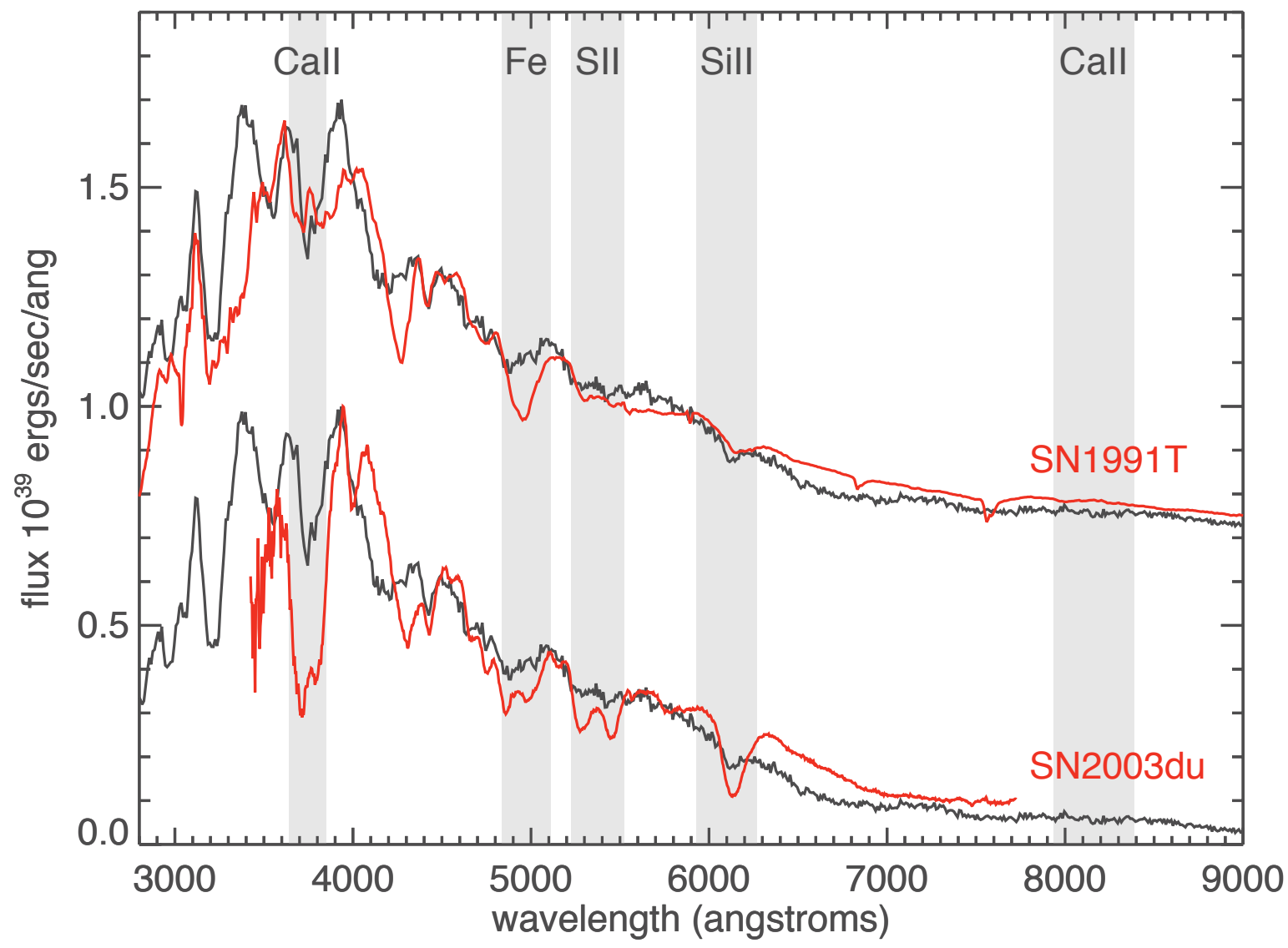

Fig. 11.- Maximum light synthetic spectrum of the entire Model 9C (shell plus core; black lines) compared to observed spectra. The model lacks the strong silicon, sulfur and calcium lines seen in the normal Type Ia SN 2003du (bottom red line, Stanishev et al. (2007)), and resembles more closely the peculiar Type Ia SN 1991T (top red line, Filippenko et al. (1992)). 


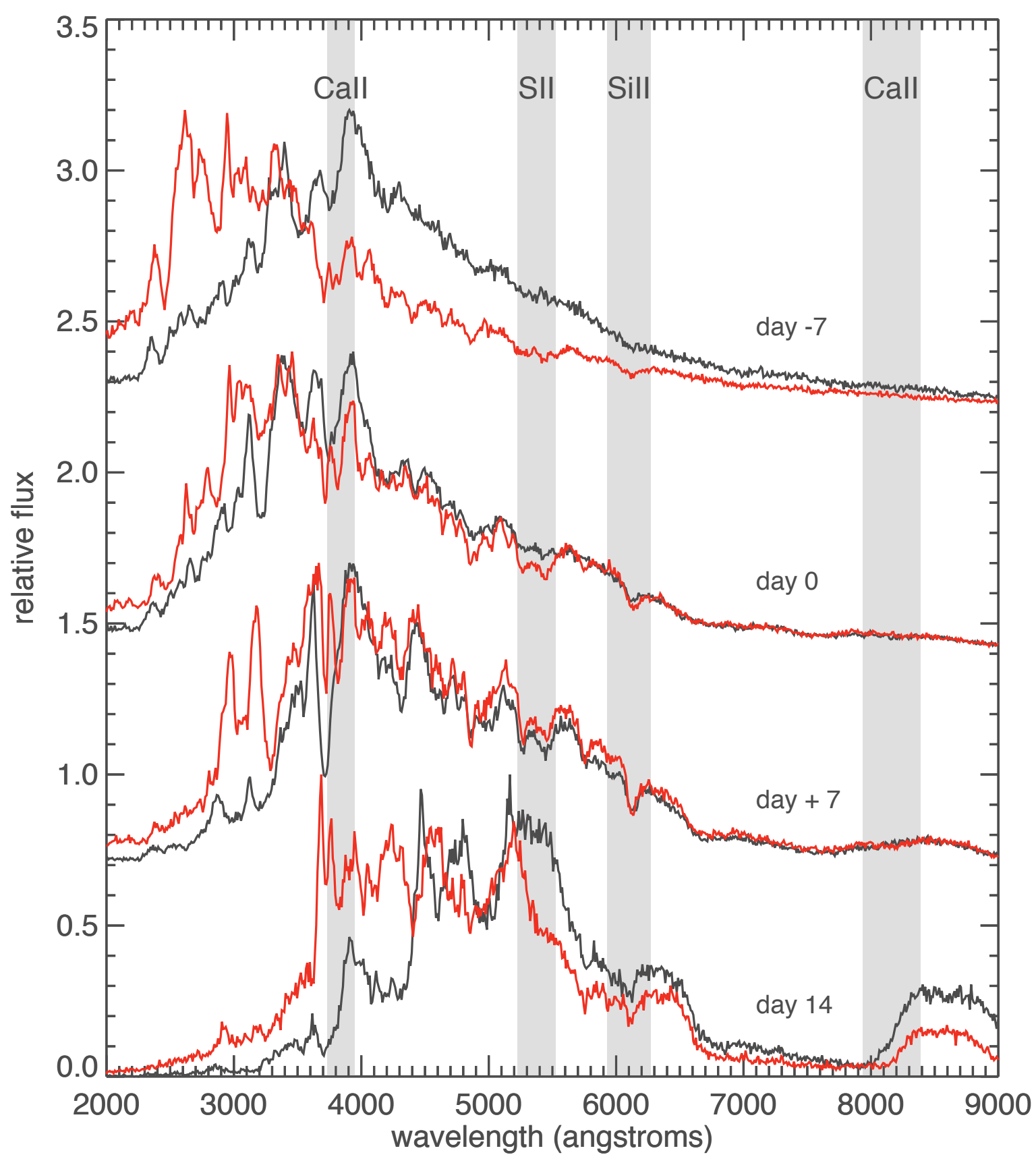

Fig. 12.- Spectral time series of Model 9C, calculated with the external burned helium shell included (black lines) and with that shell removed (red lines). Labels mark days since B-band maximum light. The presence of the shell affects both the color and line features, especially before and after maximum light. 


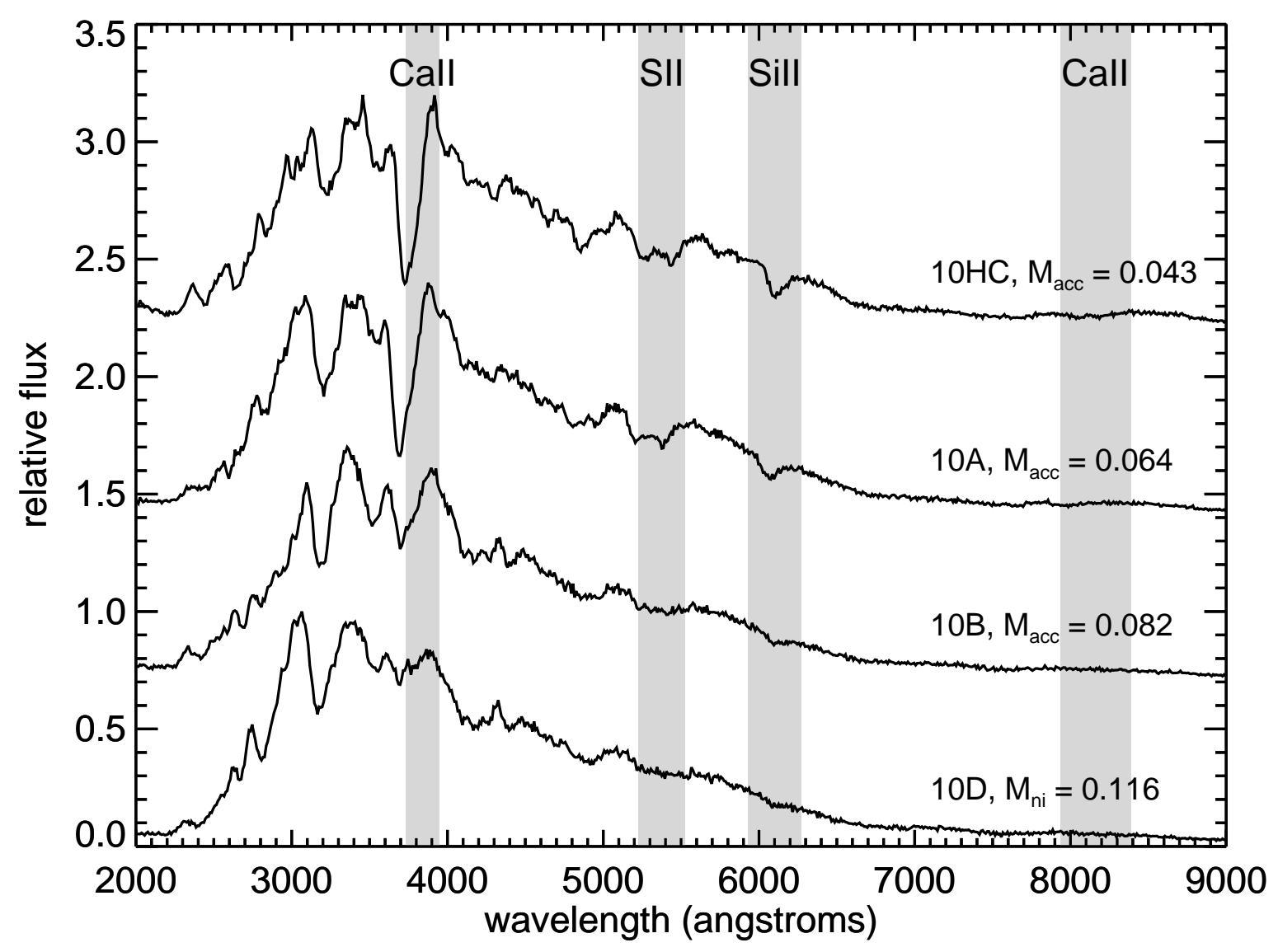

Fig. 13.- Maximum light spectra of select full star (core plus shell) explosions. These particular models all had the same core mass $\left(=1 \mathrm{M}_{\odot}\right)$ but varied in the amount of helium accreted before a detonation took place. Only the models with the lightest helium shells (Model 10HC and, to a lesser extent, Model 10A) show the IME absorption features typical of normal SNe Ia 


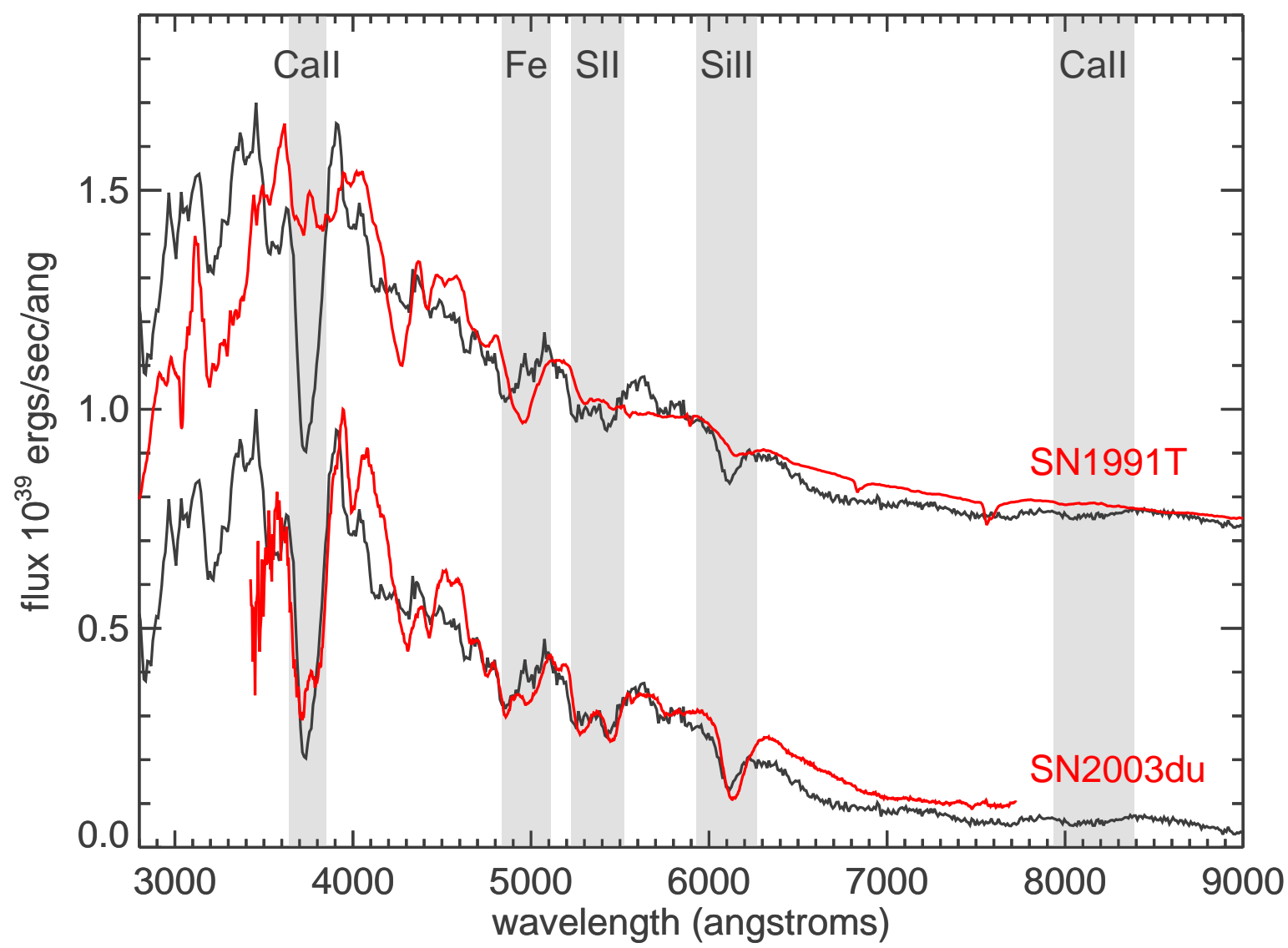

Fig. 14.- Maximum light spectrum of Model 10HC (black lines) compared to observed SNe Ia. The model shows reasonably strong absorption features from IME which are in fair agreement with the normal Type Ia SN 2003du (bottom red line) but not the peculiar SN $1991 \mathrm{~T}$ (top red line). 


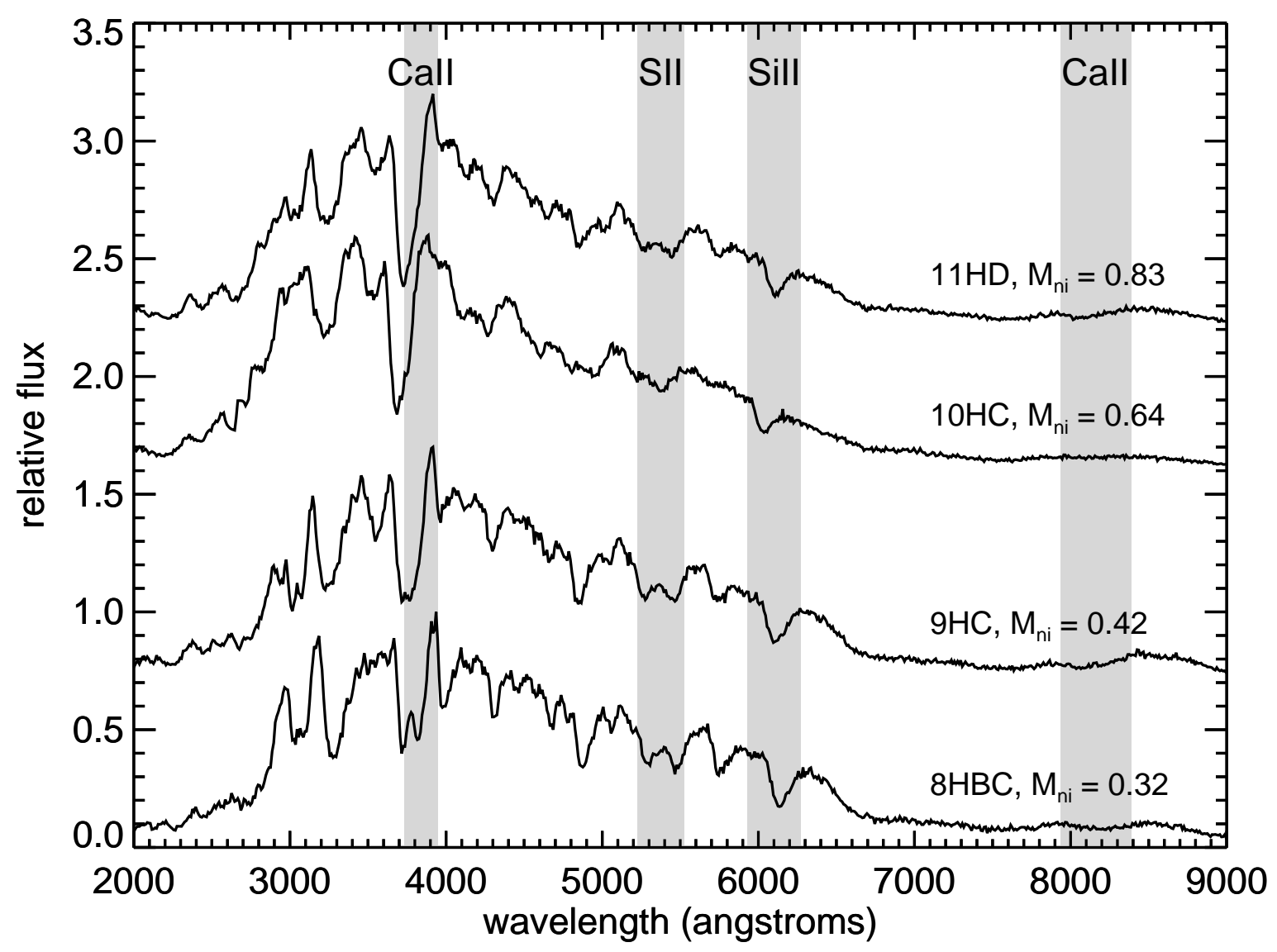

Fig. 15. - Maximum light spectra of four full star (core plus shell) explosions in which the white dwarf was "hot" - i.e., had an initial luminosity of $1 \mathrm{~L}_{\odot}$. The models vary in the initial mass of the white dwarf, and consequently the amount of ${ }^{56} \mathrm{Ni}$ produced in the explosion. In all cases, the spectra resemble normal SNe Ia, with noticeable line absorption features from IMEs (Si II, S II, and Ca II). 


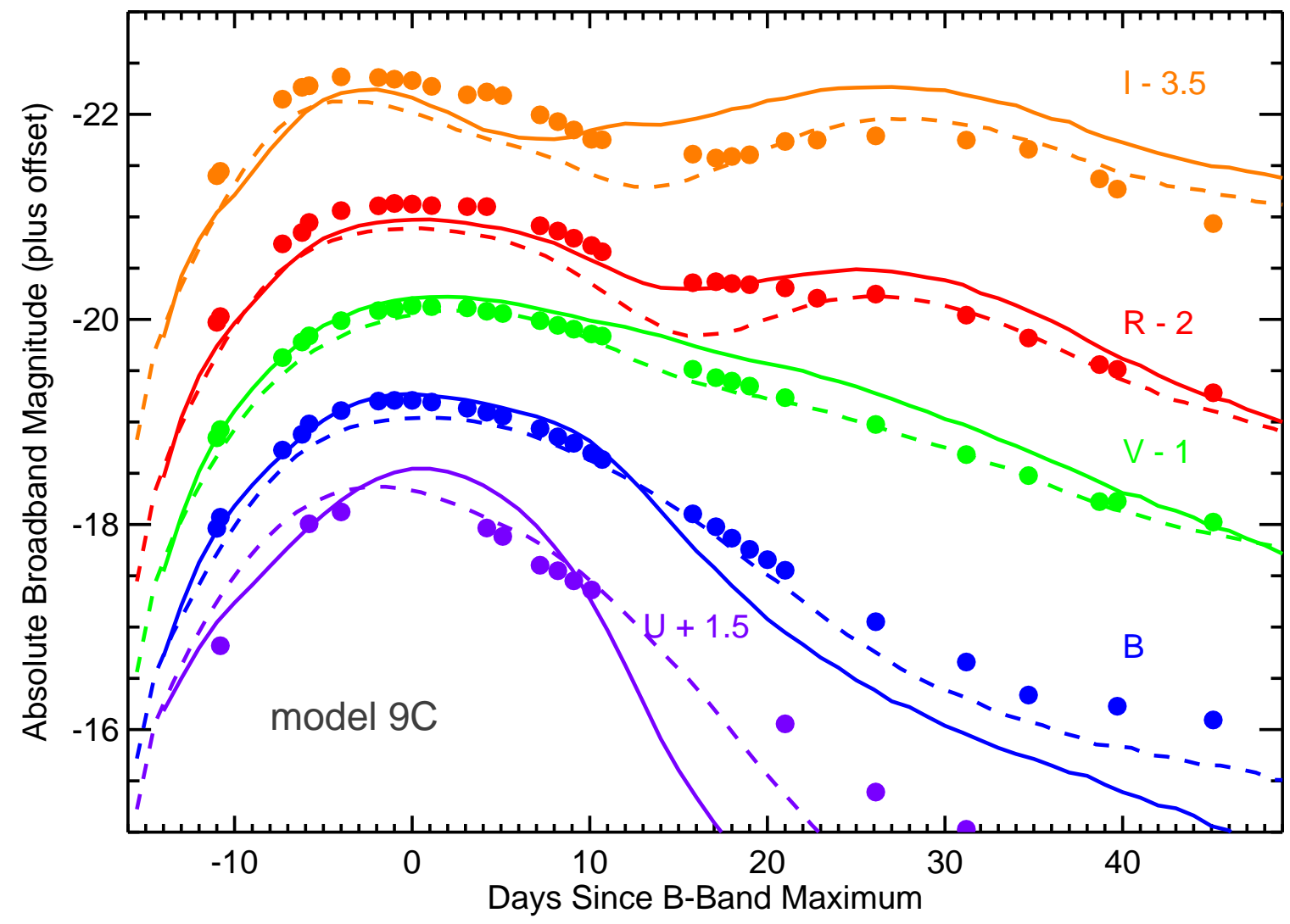

Fig. 16. - Synthetic broadband UBVRI light curves of Model 9C (solid lines), in which the both the shell and star exploded producing $0.611 \mathrm{M}_{\odot}$ of ${ }^{56} \mathrm{Ni}$. To demonstrate the effect of the outer burned helium layer, we calculated the light curves with that shell included (solid lines) and with it removed (dashed lines). The opacity of the shell causes the B-band light curves to decline after peak more rapidly than what is observed in the normal Type Ia SN 2003du (circles, Stanishev et al. (2007)). 


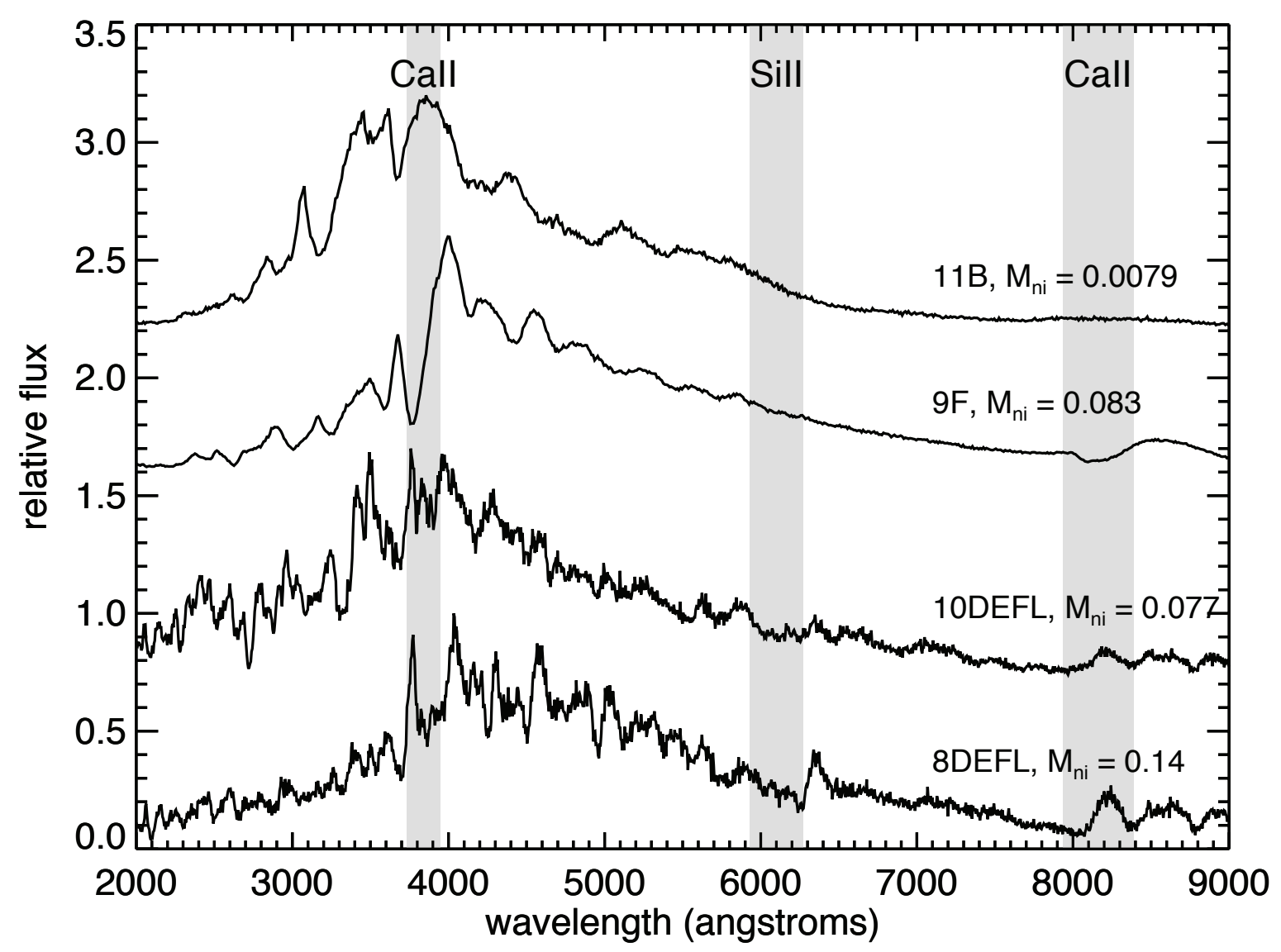

Fig. 17. - Spectra at maximum light of select shell-only explosions. The top two spectra are those of helium detonation models, which show broad, high velocity absorptions. The bottom two spectra are those of helium deflagrations, which show narrower, lower velocity absorptions. 

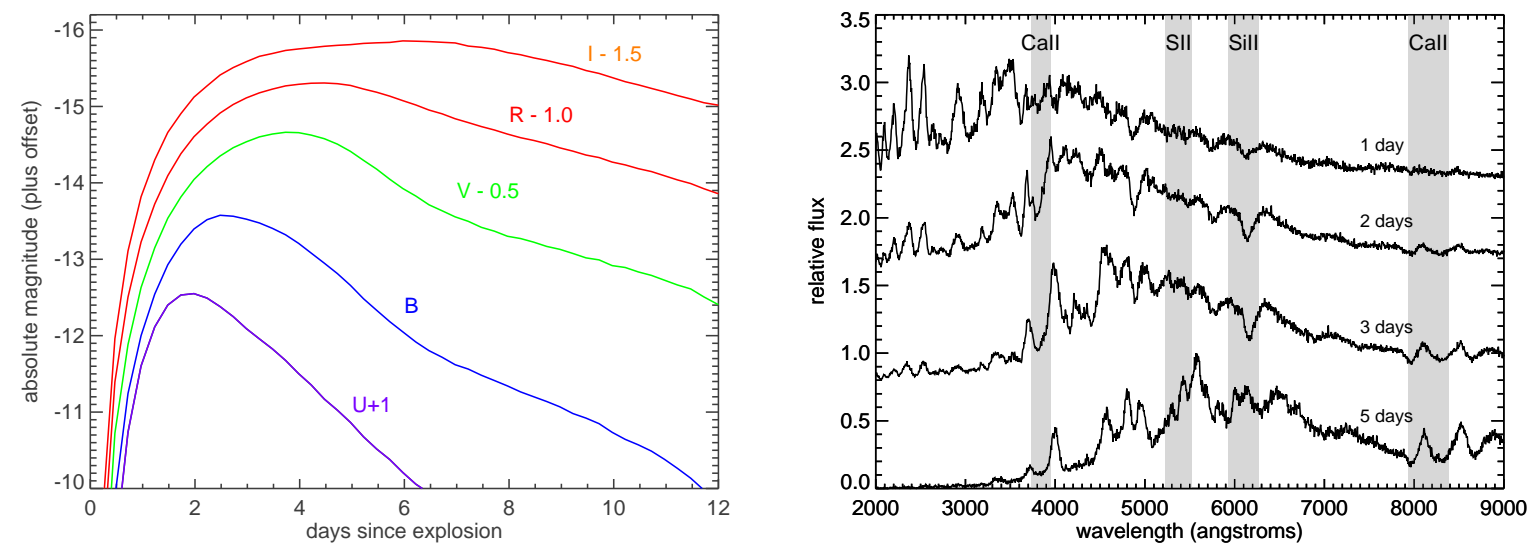

Fig. 18. - Broadband light curves (left) and spectral time series (right, marked in time since explosion) of Model $8 \mathrm{HBC} 1$, which involved the explosion of a low mass helium shell. The light curve is dim and evolves quickly; the spectra show notable features of intermediate mass elements moving at velocities near $9,000 \mathrm{~km} \mathrm{~s}^{-1}$. The observational characteristics of Models 7B1, 9A1, and 10HCD1 would probably be similar. 

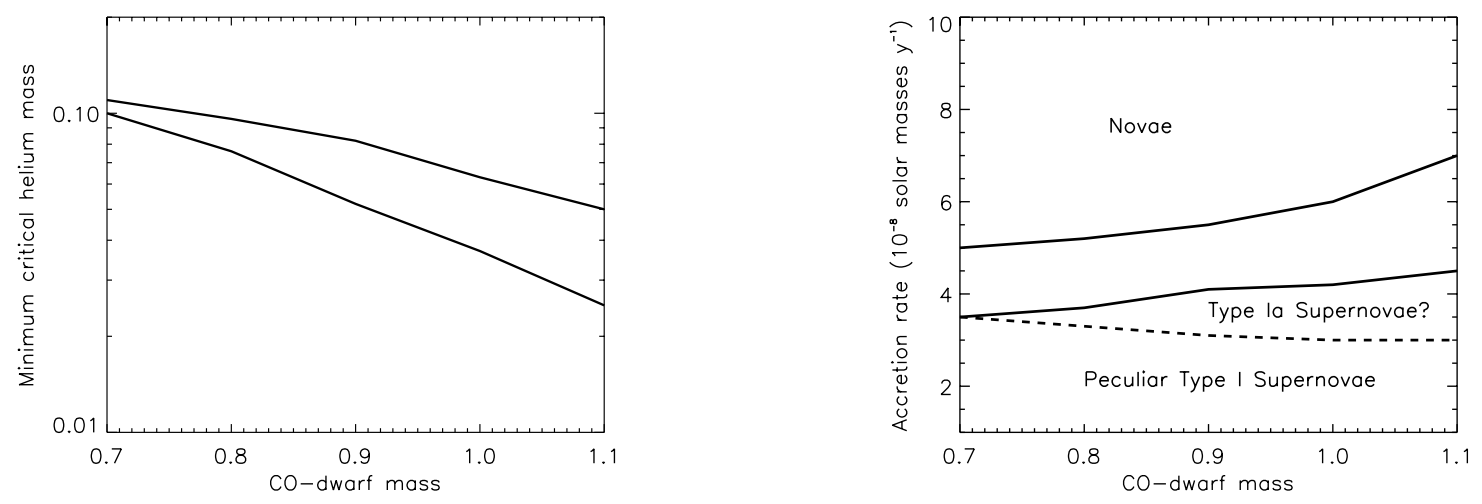

Fig. 19.- The left frame shows the minimum mass of helium required to initiate a detonation or deflagration on a CO-dwarf of given mass. The top line is interpolated from the "cold" white dwarf models (Table 1) and the bottom from the "hot" models (Table 2). Both curves lie within the range predicted by Bildsten et al. (2007) (see their Fig. 2). The frame on the right shows the expected outcome for different accretion rates on CO-dwarfs of a given mass (again interpolated from Tables 1 and 2). The top solid line is for the "cold" models and the lower solid line is for the "hot" models. Above the solid lines, nova-like outbursts are expected and not supernovae. Below the solid lines, one detonation or deflagration occurs. It is assumed that a common SN Ia supernova requires an helium shell mass less than 0.05 $\mathrm{M}_{\odot}$ or a ${ }^{56} \mathrm{Ni}$ mass in that shell of less than $0.01 \mathrm{M}_{\odot}$ (see text). None of the "cold" models satisfy this criterion. The "hot" models that do satisfy these criteria are between the lower solid and dashed lines. However, it is also for this range of conditions that both helium and carbon detonation are most questionable (due to the low density and mass of the helium shell). For larger helium shell masses and larger ${ }^{56} \mathrm{Ni}$ masses, one obtains peculiar Type I supernovae that have yet to be observed and classified. 
Table 1. Models - Cold White Dwarf Accretors

\begin{tabular}{|c|c|c|c|c|c|c|c|c|c|c|}
\hline Model & $\begin{array}{l}\text { Mass } \\
\left(\mathrm{M}_{\odot}\right)\end{array}$ & $\begin{array}{c}\mathrm{He} \\
\text { zones }^{e}\end{array}$ & $\begin{array}{c}\dot{M}_{-8} \\
\left(\mathrm{M}_{\odot} \mathrm{y}^{-1}\right)\end{array}$ & $\begin{array}{l}\mathrm{M}_{\mathrm{acc}} \\
\left(\mathrm{M}_{\odot}\right)\end{array}$ & $\begin{array}{l}\mathrm{M}_{\mathrm{ign}} \\
\left(\mathrm{M}_{\odot}\right)\end{array}$ & $\begin{array}{c}\rho_{\text {ign }} \\
\left(\mathrm{g} \mathrm{cm}^{-3}\right)\end{array}$ & $\begin{array}{c}\rho_{\text {run }} \\
\left(\mathrm{g} \mathrm{cm}^{-3}\right)\end{array}$ & $\begin{array}{c}\mathrm{M}\left({ }^{56} \mathrm{Ni}\right) \\
\left(\mathrm{M}_{\odot}\right)\end{array}$ & $\begin{array}{c}\mathrm{M}_{\text {out }}\left({ }^{56} \mathrm{Ni}\right) \\
\left(\mathrm{M}_{\odot}\right)\end{array}$ & Note \\
\hline $11 \mathrm{AA}$ & 1.1 & 86 & 8 & 0.043 & 0.018 & $1.5(6)$ & $4.9(5)$ & 0. & 0. & $\mathrm{~d}$ \\
\hline $11 \mathrm{~A}$ & 1.1 & 102 & 7 & 0.0510 & 0.0234 & $1.8(6)$ & $7.1(5)$ & 0.819 & 0.012 & $\mathrm{a}$ \\
\hline $11 \mathrm{~B}$ & 1.1 & 113 & 6 & 0.0568 & 0.0387 & $2.1(6)$ & $1.3(6)$ & 0.886 & 0.014 & b \\
\hline $11 \mathrm{C}$ & 1.1 & 124 & 5 & 0.0620 & 0.0529 & $2.3(6)$ & $1.8(6)$ & 0.848 & 0.0268 & $\mathrm{a}$ \\
\hline $11 \mathrm{D}$ & 1.1 & 176 & 4 & 0.0881 & 0.0816 & $3.8(6)$ & $3.3(6)$ & 0.927 & 0.0572 & $\mathrm{a}$ \\
\hline $11 \mathrm{E}$ & 1.1 & 266 & 3 & 0.133 & 0.133 & $8.0(6)$ & $8.0(6)$ & 1.04 & 0.0949 & $\mathrm{~b}$ \\
\hline $11 \mathrm{~F}$ & 1.1 & 398 & 2 & 0.200 & 0.200 & $2.4(7)$ & $2.4(7)$ & 1.11 & 0.162 & b \\
\hline $11 \mathrm{E} 1$ & 1.1 & 266 & 3 & 0.133 & 0.133 & $8.0(6)$ & $8.0(6)$ & 0.094 & 0.094 & c \\
\hline $11 \mathrm{~F} 1$ & 1.1 & 398 & 2 & 0.200 & 0.200 & $2.4(7)$ & $2.4(7)$ & 0.162 & 0.162 & c \\
\hline $10 \mathrm{AA}$ & 1.0 & 243 & 7 & 0.052 & 0.0262 & $1.1(6)$ & $3.8(5)$ & 0. & 0. & $\mathrm{~d}$ \\
\hline $10 \mathrm{~A}$ & 1.0 & 377 & 6 & 0.064 & 0.0315 & $1.4(6)$ & $5.4(5)$ & 0.701 & 0.013 & b \\
\hline $10 \mathrm{~B}$ & 1.0 & 163 & 5 & 0.0819 & 0.0618 & $1.8(6)$ & $1.2(6)$ & 0.745 & 0.0212 & b \\
\hline $10 \mathrm{C}$ & 1.0 & 180 & 4 & 0.0905 & 0.0744 & $2.1(6)$ & $1.6(6)$ & 0.709 & 0.0393 & $\mathrm{a}$ \\
\hline $10 \mathrm{D}$ & 1.0 & 231 & 3 & 0.116 & 0.114 & $3.1(6)$ & $2.8(6)$ & 0.785 & 0.0660 & $\mathrm{a}$ \\
\hline $10 \mathrm{E}$ & 1.0 & 354 & 2 & 0.178 & 0.178 & $6.6(6)$ & $6.6(6)$ & 0.969 & 0.125 & $\mathrm{~b}$ \\
\hline $10 \mathrm{~A} 1$ & 1.0 & 377 & 6 & 0.064 & 0.0315 & $1.4(6)$ & $5.4(5)$ & 0. & 0. & $\mathrm{c}$ \\
\hline 10B1 & 1.0 & 163 & 5 & 0.0819 & 0.0618 & $1.8(6)$ & $1.2(6)$ & 0.0138 & 0.0138 & c \\
\hline $10 \mathrm{E} 1$ & 1.0 & 354 & 2 & 0.178 & 0.178 & $6.6(6)$ & $6.6(6)$ & 0.125 & 0.125 & c \\
\hline $9 \mathrm{AA}$ & 0.9 & 159 & 6 & 0.0794 & 0.0418 & $1.1(6)$ & $4.1(5)$ & 0. & 0. & $\mathrm{~d}$ \\
\hline $9 \mathrm{~A}$ & 0.9 & 199 & 5 & 0.099 & 0.0564 & $1.5(6)$ & $6.7(5)$ & 0.488 & 0.018 & $\mathrm{a}$ \\
\hline $9 \mathrm{~B}$ & 0.9 & 215 & 4.5 & 0.109 & 0.0803 & $1.6(6)$ & $1.1(6)$ & 0.586 & 0.024 & $\mathrm{~b}$ \\
\hline $9 \mathrm{C}$ & 0.9 & 227 & 4 & 0.120 & 0.0960 & $1.8(6)$ & $1.3(6)$ & 0.611 & 0.0397 & $\mathrm{~b}$ \\
\hline $9 \mathrm{D}$ & 0.9 & 248 & 3 & 0.126 & 0.108 & $2.0(6)$ & $1.6(6)$ & 0.639 & 0.051 & $\mathrm{~b}$ \\
\hline $9 \mathrm{E}$ & 0.9 & 306 & 2 & 0.154 & 0.154 & $2.7(6)$ & $2.7(6)$ & 0.727 & 0.0833 & $\mathrm{~b}$ \\
\hline 9A1 & 0.9 & 198 & 5 & 0.099 & 0.0564 & $1.5(6)$ & $6.7(5)$ & $9.3(-5)$ & $9.3(-5)$ & $\mathrm{c}$ \\
\hline $9 \mathrm{C} 1$ & 0.9 & 227 & 4 & 0.120 & 0.0960 & $1.8(6)$ & $1.8(6)$ & 0.0397 & 0.0397 & c \\
\hline $9 \mathrm{E} 1$ & 0.9 & 306 & 2 & 0.154 & 0.154 & $2.7(6)$ & $2.7(6)$ & 0.0832 & 0.0832 & c \\
\hline $8 \mathrm{AA}$ & 0.8 & 315 & 6 & 0.0839 & 0.0539 & $7.4(5)$ & $2.9(5)$ & 0. & 0. & $\mathrm{~d}$ \\
\hline $8 \mathrm{~A}$ & 0.8 & 340 & 5 & 0.106 & 0.0650 & $1.0(6)$ & $4.5(5)$ & 0.331 & 0.011 & $\mathrm{~b}$ \\
\hline $8 \mathrm{~B}$ & 0.8 & 142 & 4 & 0.142 & 0.114 & $1.6(6)$ & $1.1(6)$ & 0.388 & 0.0460 & $\mathrm{a}$ \\
\hline $8 \mathrm{C}$ & 0.8 & 157 & 3 & 0.157 & 0.141 & $1.8(6)$ & $1.5(6)$ & 0.419 & 0.0644 & $\mathrm{a}$ \\
\hline $8 \mathrm{D}$ & 0.8 & 175 & 2 & 0.175 & 0.175 & $2.1(6)$ & $2.1(6)$ & 0.579 & 0.0833 & $\mathrm{~b}$ \\
\hline $8 \mathrm{E}$ & 0.8 & 279 & 1 & 0.280 & 0.280 & $5.9(6)$ & $5.9(6)$ & 0.841 & 0.197 & $\mathrm{~b}$ \\
\hline $8 \mathrm{~A} 1$ & 0.8 & 340 & 5 & 0.106 & 0.0650 & $1.0(6)$ & $4.5(5)$ & 0.0 & 0.0 & c \\
\hline $8 \mathrm{D} 1$ & 0.8 & 175 & 2 & 0.175 & 0.175 & $2.1(6)$ & $2.1(6)$ & 0.0833 & 0.0833 & c \\
\hline $8 \mathrm{E} 1$ & 0.8 & 279 & 1 & 0.280 & 0.280 & $5.9(6)$ & $5.9(6)$ & 0.197 & 0.197 & c \\
\hline $7 \mathrm{AA}$ & 0.7 & 99 & 6 & 0.098 & 0.098 & $5.1(5)$ & $1.5(5)$ & 0. & 0. & $\mathrm{~d}$ \\
\hline $7 \mathrm{~A}$ & 0.7 & 121 & 5 & 0.120 & 0.082 & $7.7(5)$ & $3.4(5)$ & 0.138 & $4.8(-3)$ & b \\
\hline $7 \mathrm{~B}$ & 0.7 & 153 & 4 & 0.153 & 0.105 & $1.1(6)$ & $6.0(5)$ & 0.256 & 0.015 & $\mathrm{~b}$ \\
\hline $7 \mathrm{C}$ & 0.7 & 185 & 3 & 0.185 & 0.172 & $1.6(6)$ & $1.3(6)$ & 0.262 & 0.068 & $\mathrm{a}$ \\
\hline $7 \mathrm{D}$ & 0.7 & 208 & 2 & 0.207 & 0.182 & $2.0(6)$ & $1.5(6)$ & 0.327 & 0.0928 & $\mathrm{a}$ \\
\hline $7 \mathrm{E}$ & 0.7 & 260 & 1 & 0.261 & 0.261 & $3.0(6)$ & $3.0(6)$ & 0.615 & 0.154 & b \\
\hline $7 \mathrm{~A} 1$ & 0.7 & 121 & 5 & 0.120 & 0.082 & $7.7(5)$ & $3.4(5)$ & 0. & 0. & c \\
\hline 7B1 & 0.7 & 153 & 4 & 0.153 & 0.105 & $1.1(6)$ & $6.0(5)$ & $5.6(-4)$ & $5.6(-4)$ & c \\
\hline
\end{tabular}


Table 1 - Continued

\begin{tabular}{|c|c|c|c|c|c|c|c|c|c|c|}
\hline Model & $\begin{array}{l}\text { Mass } \\
\left(\mathrm{M}_{\odot}\right)\end{array}$ & $\begin{array}{c}\mathrm{He} \\
\text { zones }^{e}\end{array}$ & $\begin{array}{c}\dot{M}_{-8} \\
\left(\mathrm{M}_{\odot} \mathrm{y}^{-1}\right)\end{array}$ & $\begin{array}{l}\mathrm{M}_{\mathrm{acc}} \\
\left(\mathrm{M}_{\odot}\right)\end{array}$ & $\begin{array}{l}\mathrm{M}_{\text {ign }} \\
\left(\mathrm{M}_{\odot}\right)\end{array}$ & $\begin{array}{c}\rho_{\text {ign }} \\
\left(\mathrm{g} \mathrm{cm}^{-3}\right)\end{array}$ & $\begin{array}{c}\rho_{\text {run }} \\
\left(\mathrm{g} \mathrm{cm}^{-3}\right)\end{array}$ & $\begin{array}{c}\mathrm{M}\left({ }^{56} \mathrm{Ni}\right) \\
\left(\mathrm{M}_{\odot}\right)\end{array}$ & $\begin{array}{c}\mathrm{M}_{\text {out }}\left({ }^{56} \mathrm{Ni}\right) \\
\left(\mathrm{M}_{\odot}\right)\end{array}$ & Note \\
\hline $7 \mathrm{E} 1$ & 0.7 & 260 & 1 & 0.261 & 0.261 & $3.0(6)$ & $3.0(6)$ & 0.154 & 0.154 & $\mathrm{c}$ \\
\hline 8DEFL & 0.8 & 157 & 3 & 0.157 & 0.141 & $1.8(6)$ & - & $6.4(-5)$ & $6.4(-5)$ & d \\
\hline 10DEFL & 1.0 & 333 & 3 & 0.0972 & 0.0777 & $2.4(6)$ & - & $9.0(-5)$ & $9.0(-5)$ & $\mathrm{d}$ \\
\hline
\end{tabular}

anwards and outwards helium detonation; whole star explodes.

${ }^{\mathrm{b}}$ Outwards helium detonation, compresses CO-core and leads to secondary detonation.

${ }^{\mathrm{c}}$ Helium detonation only; no $\mathrm{CO}$ detonation.

${ }^{\mathrm{d}}$ No prompt detonation, deflagration or convective runaway.

e All models had 375 zones in the $\mathrm{CO}$ core plus the number of helium zones indicated. 
Table 2. Models - Hot White Dwarf Accretor

\begin{tabular}{|c|c|c|c|c|c|c|c|c|c|c|}
\hline Model & $\begin{array}{l}\text { Mass } \\
\left(\mathrm{M}_{\odot}\right)\end{array}$ & $\begin{array}{c}\mathrm{He} \\
\text { zones }^{e}\end{array}$ & $\dot{M}_{-8}$ & $\begin{array}{l}\mathrm{M}_{\mathrm{acc}} \\
\left(\mathrm{M}_{\odot}\right)\end{array}$ & $\begin{array}{l}\mathrm{M}_{\text {ign }} \\
\left(\mathrm{M}_{\odot}\right)\end{array}$ & $\begin{array}{c}\rho_{\text {ign }} \\
\left(\mathrm{g} \mathrm{cm}^{-3}\right)\end{array}$ & $\begin{array}{c}\rho_{\text {run }} \\
\left(\mathrm{g} \mathrm{cm}^{-3}\right)\end{array}$ & $\begin{array}{c}\mathrm{M}\left({ }^{56} \mathrm{Ni}\right) \\
\left(\mathrm{M}_{\odot}\right)\end{array}$ & $\begin{array}{c}\mathrm{M}_{\text {out }}\left({ }^{56} \mathrm{Ni}\right) \\
\left(\mathrm{M}_{\odot}\right)\end{array}$ & Note \\
\hline $11 \mathrm{HC}$ & 1.1 & 149 & 5 & 0.015 & 0.015 & $2.6(5)$ & $2.6(5)$ & 0. & 0. & d \\
\hline $11 \mathrm{HD}$ & 1.1 & 227 & 4 & 0.027 & 0.026 & $8.7(5)$ & $6.6(5)$ & 0.826 & $2.9(-5)$ & $\mathrm{b}$ \\
\hline 11HD1 & 1.1 & 227 & 4 & 0.027 & 0.026 & $8.7(5)$ & $6.6(5)$ & $3(-6)$ & $3(-6)$ & c \\
\hline $10 \mathrm{HB}$ & 1.0 & 129 & 5 & 0.0220 & 0.0220 & $6.0(4)$ & $6.0(4)$ & 0. & 0. & d \\
\hline $10 \mathrm{HBC}$ & 1.0 & 167 & 4.5 & 0.0288 & 0.0288 & $3.6(5)$ & $3.6(5)$ & 0. & 0. & d \\
\hline $10 \mathrm{HC}$ & 1.0 & 143 & 4 & 0.0445 & 0.0426 & $9.2(5)$ & $6.6(5)$ & 0.636 & $8.1(-5)$ & $\mathrm{b}$ \\
\hline 10HCD & 1.0 & 263 & 3.5 & 0.0638 & 0.0587 & $1.3(6)$ & $1.1(6)$ & 0.618 & $8.5(-3)$ & $\mathrm{a}$ \\
\hline 10HD & 1.0 & 277 & 3 & 0.0772 & 0.0735 & $1.7(6)$ & $1.4(6)$ & 0.663 & 0.025 & $\mathrm{a}$ \\
\hline $10 \mathrm{HC} 1$ & 1.0 & 143 & 4 & 0.0445 & 0.0426 & $9.2(5)$ & $6.6(5)$ & $2.1(-5)$ & $2.1(-5)$ & c \\
\hline 10HCD1 & 1.0 & 263 & 3.5 & 0.0638 & 0.0587 & $1.3(6)$ & $1.1(6)$ & $4.7(-3)$ & $4.7(-3)$ & c \\
\hline $9 \mathrm{HB}$ & 0.9 & 113 & 5 & 0.028 & 0.028 & $1.0(5)$ & $1.0(5)$ & 0. & 0. & d \\
\hline $9 \mathrm{HBC}$ & 0.9 & 142 & 4.5 & 0.037 & 0.037 & $2.0(5)$ & $2.0(5)$ & 0. & 0. & d \\
\hline $9 \mathrm{HC}$ & 0.9 & 183 & 4 & 0.055 & 0.054 & $6.8(5)$ & $4.9(5)$ & 0.423 & 0. & $\mathrm{~b}$ \\
\hline $9 \mathrm{HD}$ & 0.9 & 273 & 3 & 0.108 & 0.107 & $1.6(6)$ & $1.3(6)$ & 0.510 & 0.035 & $\mathrm{a}$ \\
\hline $8 \mathrm{HA}$ & 0.8 & 124 & 5 & 0.033 & 0.033 & $9.3(4)$ & $9.3(4)$ & 0. & 0. & $\mathrm{~d}$ \\
\hline $8 \mathrm{HB}$ & 0.8 & 187 & 4 & 0.059 & 0059 & $2.7(5)$ & $2.7(5)$ & 0. & 0. & d \\
\hline $8 \mathrm{HBC}$ & 0.8 & 254 & 3.5 & 0.097 & 0.0865 & $8.9(5)$ & $6.2(5)$ & 0.317 & $7(-4)$ & $\mathrm{b}$ \\
\hline $8 \mathrm{HC}$ & 0.8 & 319 & 3 & 0.139 & 0.127 & $1.5(6)$ & $1.2(6)$ & 0.364 & 0.043 & $\mathrm{a}$ \\
\hline $8 \mathrm{HBC} 1$ & 0.8 & 254 & 3.5 & 0.097 & 0.0865 & $8.9(5)$ & $6.2(5)$ & $1.5(-4)$ & $1.5(-4)$ & c \\
\hline
\end{tabular}

anwards and outwards helium detonation; whole star explodes.

${ }^{\mathrm{b}}$ Outwards helium detonation, compresses CO-core and leads to secondary detonation.

${ }^{\mathrm{c}}$ Helium detonation only; no $\mathrm{CO}$ detonation.

${ }^{\mathrm{d}}$ Convective runaway - helium nova

e All models had 375 zones in the CO core plus the number of helium zones indicated. 
Table 3. Conditions at Ignition - $10^{46} \mathrm{erg} \mathrm{s}^{-1}$ and $10^{47} \mathrm{erg} \mathrm{s}^{-1}$

\begin{tabular}{|c|c|c|c|c|}
\hline Model & $\begin{array}{c}\mathrm{T}_{46} \\
\left(10^{8} \mathrm{~K}\right)\end{array}$ & $\begin{array}{l}\rho_{46} \\
\left(10^{6} \mathrm{~g} \mathrm{~cm}^{-3}\right)\end{array}$ & $\begin{array}{c}\mathrm{T}_{46} \\
\left(10^{8} \mathrm{~K}\right)\end{array}$ & $\begin{array}{c}\rho_{46} \\
\left(10^{6} \mathrm{~g} \mathrm{~cm}^{-3}\right)\end{array}$ \\
\hline $11 \mathrm{HC}$ & 3.37 & 0.378 & $\ldots$ & $\ldots$ \\
\hline 11HD & 2.86 & 0.728 & 3.69 & 0.660 \\
\hline $10 \mathrm{HB}$ & 3.41 & 0.300 & $\ldots$ & $\ldots$ \\
\hline $10 \mathrm{HBC}$ & 3.10 & 0.438 & $\ldots$ & $\ldots$ \\
\hline $10 \mathrm{HC}$ & 2.81 & 0.717 & 3.51 & 0.655 \\
\hline $10 \mathrm{HCD}$ & 2.56 & 1.12 & 3.05 & 1.07 \\
\hline 10HD & 2.37 & 1.50 & 2.93 & 1.44 \\
\hline $9 \mathrm{HB}$ & 3.60 & 0.204 & $\ldots$ & $\ldots$ \\
\hline 9HBC & 3.17 & 0.323 & $\ldots$ & $\ldots$ \\
\hline $9 \mathrm{HC}$ & 2.80 & 0.558 & 3.62 & 0.487 \\
\hline $9 \mathrm{HD}$ & 2.36 & 1.40 & 2.88 & 1.34 \\
\hline $8 \mathrm{HA}$ & 4.42 & 0.0933 & $\ldots$ & $\ldots$ \\
\hline $8 \mathrm{HB}$ & 3.00 & 0.362 & $\ldots$ & $\ldots$ \\
\hline $8 \mathrm{HBC}$ & 2.63 & 0.690 & 3.28 & 0.625 \\
\hline $8 \mathrm{HC}$ & 2.34 & 1.26 & 2.87 & 1.20 \\
\hline $11 \mathrm{AA}$ & 3.07 & 0.572 & $\ldots$ & $\ldots$ \\
\hline $11 \mathrm{~A}$ & 2.81 & 0.789 & 3.72 & 0.713 \\
\hline $11 \mathrm{~B}$ & 2.48 & 1.35 & 3.14 & 1.29 \\
\hline $11 \mathrm{C}$ & 2.34 & 1.82 & 2.81 & 1.77 \\
\hline $11 \mathrm{D}$ & 2.09 & 3.35 & 2.46 & 3.29 \\
\hline $11 \mathrm{E}$ & 1.84 & 8.10 & 2.10 & 8.04 \\
\hline $11 \mathrm{~F}$ & 1.58 & 23.6 & 1.82 & 23.5 \\
\hline $10 \mathrm{AA}$ & 3.09 & 0.460 & $\ldots$ & $\ldots$ \\
\hline $10 \mathrm{~A}$ & 2.92 & 0.603 & 3.73 & 0.537 \\
\hline $10 \mathrm{~B}$ & 2.44 & 1.29 & 2.96 & 1.24 \\
\hline $10 \mathrm{C}$ & 2.31 & 1.64 & 2.78 & 1.59 \\
\hline $10 \mathrm{D}$ & 2.10 & 2.89 & 2.48 & 2.84 \\
\hline $10 \mathrm{E}$ & 1.81 & 6.53 & 2.13 & 6.63 \\
\hline $9 \mathrm{AA}$ & 2.95 & 0.487 & $\ldots$ & $\ldots$ \\
\hline $9 \mathrm{~A}$ & 2.69 & 0.742 & 3.46 & 0.672 \\
\hline $9 \mathrm{~B}$ & 2.49 & 1.12 & 3.03 & 1.06 \\
\hline $9 \mathrm{C}$ & 2.35 & 1.39 & 2.86 & 1.33 \\
\hline $9 \mathrm{D}$ & 2.27 & 1.64 & 2.77 & 1.58 \\
\hline $9 \mathrm{E}$ & 2.09 & 2.75 & 2.44 & 2.70 \\
\hline $8 \mathrm{AA}$ & 3.00 & 0.376 & $\ldots$ & $\ldots$ \\
\hline $8 \mathrm{~A}$ & 2.86 & 0.522 & 3.62 & 0.455 \\
\hline $8 \mathrm{~B}$ & 2.42 & 1.14 & 2.93 & 1.09 \\
\hline $8 \mathrm{C}$ & 2.25 & 1.53 & 2.70 & 1.48 \\
\hline $8 \mathrm{D}$ & 2.12 & 2.12 & 2.57 & 2.06 \\
\hline $8 \mathrm{E}$ & 1.84 & 5.96 & 2.13 & 5.89 \\
\hline
\end{tabular}


Table 3-Continued

\begin{tabular}{ccccc}
\hline \hline Model & $\begin{array}{c}\mathrm{T}_{46} \\
\left(10^{8} \mathrm{~K}\right)\end{array}$ & $\begin{array}{c}\rho_{46} \\
\left(10^{6} \mathrm{~g} \mathrm{~cm}^{-3}\right)\end{array}$ & $\begin{array}{c}\mathrm{T}_{46} \\
\left(10^{8} \mathrm{~K}\right)\end{array}$ & $\begin{array}{c}\rho_{46} \\
\left(10^{6} \mathrm{~g} \mathrm{~cm}^{-3}\right)\end{array}$ \\
\hline 7AA & 3.10 & 0.291 & $\ldots$ & $\ldots$ \\
$7 \mathrm{~A}$ & 2.83 & 0.423 & 3.76 & 0.339 \\
$7 \mathrm{~B}$ & 2.58 & 0.669 & 3.26 & 0.598 \\
$7 \mathrm{C}$ & 2.27 & 1.36 & 2.75 & 1.30 \\
$7 \mathrm{D}$ & 2.23 & 1.60 & 2.66 & 1.54 \\
$7 \mathrm{E}$ & 2.01 & 3.02 & 2.35 & 2.96 \\
\hline
\end{tabular}

Table 4. Explosion Characteristics

\begin{tabular}{|c|c|c|c|c|c|c|c|}
\hline Model & $\begin{array}{l}\mathrm{KE} \\
(\mathrm{erg})\end{array}$ & $\begin{array}{c}\text { Remnant } \\
\quad\left(\mathrm{M}_{\odot}\right)\end{array}$ & $\begin{array}{l}\text { Eject } \\
\left(\mathrm{M}_{\odot}\right)\end{array}$ & Model & $\begin{array}{l}\mathrm{KE} \\
(\mathrm{erg})\end{array}$ & $\begin{array}{l}\text { Remnant } \\
\qquad\left(\mathrm{M}_{\odot}\right)\end{array}$ & $\begin{array}{c}\text { Eject } \\
\left(\mathrm{M}_{\odot}\right)\end{array}$ \\
\hline $11 \mathrm{~A}$ & $1.4(51)$ & 0 & 1.15 & $9 \mathrm{HC}$ & $1.1(51)$ & 0 & 0.96 \\
\hline $11 \mathrm{~B}$ & $1.5(51)$ & 0 & 1.16 & $9 \mathrm{D}$ & $1.3(51)$ & 0 & 1.02 \\
\hline $11 \mathrm{C}$ & $1.5(51)$ & 0 & 1.16 & $9 \mathrm{HD}$ & $1.2(51)$ & 0 & 1.01 \\
\hline $11 \mathrm{D}$ & $1.5(51)$ & 0 & 1.19 & $9 \mathrm{E}$ & $1.4(51)$ & 0 & 1.05 \\
\hline $11 \mathrm{HD}$ & $1.4(51)$ & 0 & 1.13 & $9 \mathrm{~A} 1$ & $3.4(49)$ & 0.95 & 0.049 \\
\hline $11 \mathrm{E}$ & $1.7(51)$ & 0 & 1.23 & $9 \mathrm{C} 1$ & $1.9(50)$ & 0.90 & 0.12 \\
\hline $11 \mathrm{~F}$ & $1.9(51)$ & 0 & 1.30 & $9 \mathrm{E} 1$ & $2.4(50)$ & 0.90 & 0.15 \\
\hline 11HD1 & $9.7(48)$ & 1.11 & 0.017 & $8 \mathrm{~A}$ & $9.9(50)$ & 0 & 0.91 \\
\hline $11 \mathrm{E} 1$ & $2.1(50)$ & 1.11 & 0.12 & $8 \mathrm{~B}$ & $1.2(51)$ & 0 & 0.94 \\
\hline \multirow[t]{3}{*}{$11 \mathrm{~F} 1$} & $3.3(50)$ & 1.10 & 0.20 & $8 \mathrm{HBC}$ & $9.7(50)$ & 0 & 0.90 \\
\hline & & & & $8 \mathrm{C}$ & $1.2(51)$ & 0 & 0.96 \\
\hline & & & & $8 \mathrm{HC}$ & $1.1(51)$ & 0 & 0.94 \\
\hline $10 \mathrm{~A}$ & $1.3(51)$ & 0 & 1.06 & $8 \mathrm{D}$ & $1.3(51)$ & 0 & 0.97 \\
\hline $10 \mathrm{~B}$ & $1.4(51)$ & 0 & 1.08 & $8 \mathrm{E}$ & $1.7(51)$ & 0 & 1.08 \\
\hline $10 \mathrm{C}$ & $1.4(51)$ & 0 & 1.09 & & & & \\
\hline $10 \mathrm{HC}$ & $1.2(51)$ & 0 & 1.05 & $8 \mathrm{~A} 1$ & $3.6(49)$ & 0.85 & 0.056 \\
\hline 10HCD & $1.2(51)$ & 0 & 1.07 & $8 \mathrm{HBC} 1$ & $6.8(49)$ & 0.81 & 0.087 \\
\hline $10 \mathrm{D}$ & $1.4(51)$ & 0 & 1.12 & $8 \mathrm{D} 1$ & $2.7(50)$ & 0.78 & 0.19 \\
\hline $10 \mathrm{HD}$ & $1.3(51)$ & 0 & 1.08 & $8 \mathrm{E} 1$ & $5.3(50)$ & 0.77 & 0.31 \\
\hline \multirow[t]{2}{*}{$10 \mathrm{E}$} & $1.7(51)$ & 0 & 1.18 & & & & \\
\hline & & & & $7 \mathrm{~A}$ & $7.5(50)$ & 0 & 0.82 \\
\hline $10 \mathrm{~A} 1$ & 1.1(49) & 1.04 & 0.024 & $7 \mathrm{~B}$ & $9.4(50)$ & 0 & 0.85 \\
\hline 10B1 & $6.2(49)$ & 1.03 & 0.05 & $7 \mathrm{C}$ & $1.0(51)$ & 0 & 0.89 \\
\hline 10HC1 & $2.3(49)$ & 1.01 & 0.035 & $7 \mathrm{D}$ & $1.1(51)$ & 0 & 0.91 \\
\hline \multirow[t]{2}{*}{ 10HCD1 } & $5.4(49)$ & 1.01 & 0.057 & $7 \mathrm{E}$ & $1.4(51)$ & 0 & 0.96 \\
\hline & & & & $7 \mathrm{~A} 1$ & $3.7(49)$ & 0.74 & 0.081 \\
\hline \multirow[t]{2}{*}{$10 \mathrm{E} 1$} & $3.1(50)$ & 0.99 & 0.19 & 7B1 & $1.0(50)$ & 0.75 & 0.10 \\
\hline & & & & $7 \mathrm{E} 1$ & $4.6(50)$ & 0.69 & 0.27 \\
\hline $9 \mathrm{~A}$ & $1.2(51)$ & 0 & 1.00 & & & & \\
\hline $9 \mathrm{~B}$ & $1.2(51)$ & 0 & 1.01 & 10DEFL & $2.3(49)$ & 1.03 & 0.069 \\
\hline $9 \mathrm{C}$ & $1.3(51)$ & 0 & 1.02 & 8DEFL & $3.0(49)$ & 0.82 & 0.14 \\
\hline
\end{tabular}


Table 5. Radioactivities Produced in the Explosions (Solar Masses)

\begin{tabular}{|c|c|c|c|c|c|c|c|}
\hline Model & ${ }^{44} \mathrm{Ti}$ & ${ }^{48} \mathrm{Cr}$ & ${ }^{52} \mathrm{Fe}$ & ${ }^{55} \mathrm{Co}$ & ${ }^{56} \mathrm{Ni}$ & ${ }^{57} \mathrm{Ni}$ & ${ }^{59} \mathrm{Cu}+{ }^{59} \mathrm{Ni}$ \\
\hline $11 \mathrm{~A}$ & $1.2(-3)$ & $3.1(-3)$ & $6.8(-3)$ & $1.2(-3)$ & $8.2(-1)$ & $1.9(-2)$ & $6.5(-4)$ \\
\hline $11 \mathrm{~B}$ & $6.2(-4)$ & $2.8(-3)$ & $9.1(-3)$ & $1.4(-3)$ & $8.9(-1)$ & $2.0(-2)$ & $6.2(-4)$ \\
\hline $11 \mathrm{C}$ & $2.7(-4)$ & $1.5(-3)$ & $7.4(-3)$ & $1.3(-3)$ & $8.5(-1)$ & $2.1(-2)$ & $9.1(-4)$ \\
\hline $11 \mathrm{D}$ & $1.6(-4)$ & $8.3(-4)$ & $5.6(-3)$ & $1.1(-3)$ & $9.3(-1)$ & $2.4(-2)$ & $9.0(-4)$ \\
\hline $11 \mathrm{HD}$ & $4.6(-4)$ & $4.1(-4)$ & $6.1(-3)$ & $1.2(-3)$ & $8.3(-1)$ & $1.7(-2)$ & $4.7(-4)$ \\
\hline $11 \mathrm{E}$ & $9.6(-5)$ & $4.4(-4)$ & $3.1(-3)$ & $5.0(-4)$ & $1.0(00)$ & $2.9(-2)$ & $1.2(-3)$ \\
\hline $11 \mathrm{~F}$ & $4.4(-5)$ & $1.1(-4)$ & $5.8(-4)$ & $9.0(-4)$ & $1.1(00)$ & $3.8(-2)$ & $1.3(-3)$ \\
\hline 11HD1 & $4.3(-4)$ & $5.8(-5)$ & $\ldots$ & $\ldots$ & $\ldots$ & $\ldots$ & $\ldots$ \\
\hline $11 \mathrm{E} 1$ & $8.4(-5)$ & $2.9(-4)$ & $5.2(-4)$ & $3.0(-5)$ & $9.4(-2)$ & $4.0(-3)$ & $4.3(-4)$ \\
\hline $11 \mathrm{~F} 1$ & $3.8(-5)$ & $1.3(-4)$ & $2.2(-4)$ & $1.2(-5)$ & $1.6(-1)$ & $5.7(-3)$ & $3.1(-4)$ \\
\hline $10 \mathrm{~A}$ & $2.6(-4)$ & $5.8(-4)$ & $7.7(-3)$ & $1.5(-3)$ & $7.0(-1)$ & $1.4(-2)$ & $7.2(-4)$ \\
\hline $10 \mathrm{~B}$ & $8.4(-4)$ & $3.8(-3)$ & $1.3(-2)$ & $1.7(-3)$ & $7.4(-1)$ & $1.7(-2)$ & $5.3(-4)$ \\
\hline $10 \mathrm{C}$ & $4.5(-4)$ & $2.3(-3)$ & $1.0(-2)$ & $1.7(-3)$ & $7.1(-1)$ & $1.8(-2)$ & $8.3(-4)$ \\
\hline $10 \mathrm{HC}$ & $1.1(-3)$ & $5.8(-4)$ & $7.4(-3)$ & $1.5(-3)$ & $6.4(-1)$ & $1.2(-2)$ & $3.0(-4)$ \\
\hline $10 \mathrm{HCD}$ & $1.3(-3)$ & $5.1(-3)$ & $1.7(-2)$ & $2.2(-3)$ & $6.2(-1)$ & $1.3(-2)$ & $3.8(-4)$ \\
\hline $10 \mathrm{D}$ & $2.6(-4)$ & $1.3(-3)$ & $7.7(-3)$ & $1.4(-3)$ & $7.9(-1)$ & $2.0(-2)$ & $8.0(-4)$ \\
\hline $10 \mathrm{HD}$ & $6.4(-4)$ & $3.0(-3)$ & $1.2(-2)$ & $1.8(-3)$ & $6.6(-1)$ & $1.6(-2)$ & $5.3(-4)$ \\
\hline $10 \mathrm{E}$ & $1.4(-4)$ & $6.5(-4)$ & $4.2(-3)$ & $7.2(-4)$ & $9.7(-1)$ & $2.6(-2)$ & $1.2(-3)$ \\
\hline $10 \mathrm{~A} 1$ & $1.9(-4)$ & $1.2(-5)$ & $\ldots$ & $\ldots$ & $\ldots$ & $\ldots$ & $\ldots$ \\
\hline 10B1 & $7.7(-4)$ & $3.3(-3)$ & $6.5(-3)$ & $4.2(-4)$ & $1.4(-2)$ & $1.7(-3)$ & $4.2(-5)$ \\
\hline $10 \mathrm{HC} 1$ & $1.1(-3)$ & $1.7(-4)$ & $4.1(-5)$ & $\ldots$ & $2.1(-5)$ & $\ldots$ & $\ldots$ \\
\hline 10HCD1 & $1.7(-3)$ & $7.6(-3)$ & $7.1(-3)$ & $4.9(-4)$ & $4.7(-3)$ & $6.1(-4)$ & $3.4(-5)$ \\
\hline $10 \mathrm{E} 1$ & $1.2(-4)$ & $4.5(-4)$ & $8.3(-4)$ & $4.9(-5)$ & $1.2(-1)$ & $5.4(-3)$ & $5.0(-4)$ \\
\hline $9 \mathrm{~A}$ & $3.4(-3)$ & $6.5(-3)$ & $1.0(-2)$ & $1.7(-3)$ & $4.9(-1)$ & $1.0(-2)$ & $2.8(-4)$ \\
\hline $9 \mathrm{~B}$ & $1.5(-3)$ & $7.0(-3)$ & $1.6(-2)$ & $2.1(-3)$ & $5.9(-1)$ & $1.3(-2)$ & $5.3(-4)$ \\
\hline $9 \mathrm{C}$ & $9.6(-4)$ & $4.4(-3)$ & $1.3(-2)$ & $1.8(-3)$ & $6.1(-1)$ & $1.5(-2)$ & $4.8(-5)$ \\
\hline $9 \mathrm{HC}$ & $3.6(-4)$ & $4.1(-4)$ & $8.0(-3)$ & $1.7(-3)$ & $4.2(-1)$ & $6.3(-3)$ & $1.4(-4)$ \\
\hline $9 \mathrm{D}$ & $6.5(-4)$ & $3.3(-3)$ & $1.1(-2)$ & $1.6(-3)$ & $6.4(-1)$ & $1.7(-2)$ & $7.3(-4)$ \\
\hline 9HD & $9.3(-4$ & $4.2(-3)$ & $1.5(-2)$ & $2.1(-3)$ & $5.1(-1)$ & $1.3(-2)$ & $4.1(-4)$ \\
\hline $9 \mathrm{E}$ & $4.1(-4)$ & $2.0(-3)$ & $8.5(-3)$ & $1.4(-3)$ & $7.3(-1)$ & $2.0(-2)$ & $7.1(-4)$ \\
\hline $9 \mathrm{~A} 1$ & $2.2(-3)$ & $5.9(-4)$ & $1.5(-4)$ & $1.0(-5)$ & $9.3(-5)$ & $1.3(-5)$ & $\ldots$ \\
\hline $9 \mathrm{C} 1$ & $9.3(-4)$ & $4.1(-3)$ & $7.0(-3)$ & $4.9(-4)$ & $4.0(-2)$ & $4.2(-3)$ & $2.0(-4)$ \\
\hline $9 \mathrm{E} 1$ & $3.9(-4)$ & $1.7(-3)$ & $3.1(-3)$ & $2.1(-4)$ & $8.3(-2)$ & $7.2(-3)$ & $4.3(-4)$ \\
\hline $8 \mathrm{~A}$ & $5.1(-4)$ & $1.4(-3)$ & $1.0(-2)$ & $2.0(-3)$ & $3.3(-1)$ & $5.3(-3)$ & $3.8(-4)$ \\
\hline $8 \mathrm{~B}$ & $1.7(-3)$ & $6.4(-3)$ & $2.0(-2)$ & $2.3(-3)$ & $3.9(-1)$ & $9.4(-3)$ & $2.6(-4)$ \\
\hline $8 \mathrm{HBC}$ & $3.7(-3)$ & $1.9(-3)$ & $8.5(-3)$ & $1.7(-3)$ & $3.2(-1)$ & $4.4(-3)$ & $9.4(-5)$ \\
\hline $8 \mathrm{C}$ & $8.9(-4)$ & $4.4(-3)$ & $1.4(-2)$ & $2.0(-3)$ & $4.2(-1)$ & $1.3(-2)$ & $6.6(-4)$ \\
\hline $8 \mathrm{HC}$ & $1.5(-3)$ & $6.1(-3)$ & $1.8(-2)$ & $2.2(-3)$ & $3.6(-1)$ & $1.0(-2)$ & $2.5(-4)$ \\
\hline $8 \mathrm{D}$ & $6.4(-4)$ & $3.3(-3)$ & $1.2(-2)$ & $1.6(-3)$ & $5.8(-1)$ & $1.7(-2)$ & $7.4(-4)$ \\
\hline $8 \mathrm{E}$ & $2.2(-4)$ & $1.0(-3)$ & $5.9(-3)$ & $9.6(-4)$ & $8.4(-1)$ & $2.3(-2)$ & $1.1(-3)$ \\
\hline $8 \mathrm{~A} 1$ & $3.1(-4)$ & $1.2(-5)$ & $\ldots$ & $\ldots$ & $\ldots$ & $\ldots$ & $\ldots$ \\
\hline 8HBC1 & $3.6(-3)$ & $9.6(-4)$ & $2.5(-4)$ & $2.0(-5)$ & $1.5(-4)$ & $2.1(-5)$ & $\ldots$ \\
\hline $8 \mathrm{D} 1$ & $6.1(-4)$ & $3.0(-3)$ & $5.6(-3)$ & $3.7(-4)$ & $8.4(-2)$ & $8.2(-3)$ & $5.9(-4)$ \\
\hline $8 \mathrm{E} 1$ & $2.1(-4)$ & $8.1(-4)$ & $1.5(-3)$ & $9.4(-5)$ & $2.0(-1)$ & $8.5(-3)$ & $7.5(-4)$ \\
\hline $7 \mathrm{~A}$ & $6.5(-4)$ & $2.7(-3)$ & $9.9(-3)$ & $1.8(-3)$ & $1.4(-1)$ & $1.6(-3)$ & $2.7(-5)$ \\
\hline
\end{tabular}


Table 5-Continued

\begin{tabular}{cccccccc}
\hline \hline Model & ${ }^{44} \mathrm{Ti}$ & ${ }^{48} \mathrm{Cr}$ & ${ }^{52} \mathrm{Fe}$ & ${ }^{55} \mathrm{Co}$ & ${ }^{56} \mathrm{Ni}$ & ${ }^{57} \mathrm{Ni}$ & ${ }^{59} \mathrm{Cu}+{ }^{59} \mathrm{Ni}$ \\
\hline 7B & $5.0(-3)$ & $5.3(-3)$ & $1.1(-2)$ & $1.9(-3)$ & $2.6(-1)$ & $4.5(-3)$ & $4.2(-4)$ \\
$7 \mathrm{C}$ & $1.4(-3)$ & $6.2(-3)$ & $1.7(-2)$ & $2.0(-3)$ & $2.6(-1)$ & $9.9(-3)$ & $4.5(-4)$ \\
$7 \mathrm{D}$ & $1.0(-3)$ & $4.9(-3)$ & $1.5(-2)$ & $2.0(-3)$ & $3.3(-1)$ & $1.3(-2)$ & $8.4(-4)$ \\
$7 \mathrm{E}$ & $6.0(-4)$ & $2.6(-3)$ & $9.4(-3)$ & $1.4(-3)$ & $6.2(-1)$ & $2.0(-2)$ & $6.4(-4)$ \\
$7 \mathrm{~A} 1$ & $5.6(-5)$ & $\ldots$ & $\ldots$ & $\ldots$ & $\ldots$ & $\ldots$ & $\ldots$ \\
$7 \mathrm{~B} 1$ & $4.8(-3)$ & $3.9(-3)$ & $1.9(-3)$ & $1.2(-4)$ & $5.6(-4)$ & $5.7(-5)$ & $1.0(-5)$ \\
$7 \mathrm{E} 1$ & $5.8(-4)$ & $2.3(-3)$ & $4.2(-3)$ & $2.8(-4)$ & $1.5(-1)$ & $1.1(-2)$ & $4.2(-4)$ \\
& & & & & & \\
10DEFL & $2.5(-3)$ & $6.3(-3)$ & $1.6(-3)$ & $1.3(-3)$ & $9.0(-5)$ & $7.3(-6)$ & $4.9(-8)$ \\
8DEFL & $3.5(-3)$ & $3.7(-3)$ & $4.6(-4)$ & $1.1(-5)$ & $6.4(-5)$ & $6.7(-6)$ & $3.0(-7)$ \\
\hline
\end{tabular}


Table 6. Major Production Factors - Double Detonation - High Mass Dwarfs

\begin{tabular}{|c|c|c|c|c|c|c|c|c|c|c|c|}
\hline Species & $11 \mathrm{~A}$ & $11 \mathrm{~B}$ & $11 \mathrm{C}$ & $11 \mathrm{D}$ & $11 \mathrm{E}$ & $11 \mathrm{~F}$ & $10 \mathrm{~A}$ & $10 \mathrm{~B}$ & $10 \mathrm{C}$ & $10 \mathrm{D}$ & $10 \mathrm{E}$ \\
\hline${ }^{28} \mathrm{Si}$ & 100 & 70 & 91 & 60 & 18 & $\ldots$ & 130 & 110 & 140 & 99 & 32 \\
\hline${ }^{32} \mathrm{~S}$ & 178 & 130 & 160 & 120 & 39 & $\ldots$ & 240 & 190 & 250 & 180 & 65 \\
\hline${ }^{34} \mathrm{~S}$ & 18 & 9.2 & 22 & $\ldots$ & $\ldots$ & $\ldots$ & 23 & 22 & 24 & 22 & $\ldots$ \\
\hline${ }^{36} \mathrm{Ar}$ & 130 & 100 & 110 & 90 & 36 & $\ldots$ & 180 & 140 & 170 & 130 & 57 \\
\hline${ }^{38} \mathrm{Ar}$ & 12 & 5.9 & 14 & $\ldots$ & $\ldots$ & $\ldots$ & 14 & 12 & 18 & $\ldots$ & $\ldots$ \\
\hline${ }^{39} \mathrm{~K}$ & 27 & 19 & 11 & $\ldots$ & $\ldots$ & $\ldots$ & 62 & 27 & 19 & $\ldots$ & $\ldots$ \\
\hline${ }^{40} \mathrm{Ca}$ & 220 & 160 & 140 & 110 & 51 & $\ldots$ & 290 & 210 & 220 & 160 & 77 \\
\hline${ }^{43} \mathrm{Ca}$ & 110 & 160 & 85 & 68 & 53 & 27 & 120 & 230 & 140 & 99 & 78 \\
\hline${ }^{44} \mathrm{Ca}$ & 630 & 320 & 140 & 81 & 46 & 20 & 140 & 460 & 240 & 140 & 69 \\
\hline${ }^{45} \mathrm{Sc}$ & 6.3 & 2.3 & 1.3 & $\ldots$ & $\ldots$ & $\ldots$ & 3.7 & 2.5 & 2.1 & 1.0 & 1.2 \\
\hline${ }^{46} \mathrm{Ti}$ & 230 & 190 & 290 & 82 & 36 & $\ldots$ & 82 & 350 & 400 & 160 & 61 \\
\hline${ }^{47} \mathrm{Ti}$ & 300 & 310 & 240 & 96 & 54 & 23 & 67 & 530 & 380 & 170 & 84 \\
\hline${ }^{48} \mathrm{Ti}$ & 1100 & 980 & 510 & 280 & 140 & 44 & 220 & 1400 & 830 & 480 & 220 \\
\hline${ }^{49} \mathrm{Ti}$ & 130 & 76 & 65 & 54 & 43 & 11 & 90 & 90 & 93 & 75 & 59 \\
\hline${ }^{51} \mathrm{~V}$ & 320 & 360 & 250 & 130 & 55 & 15 & 110 & 540 & 390 & 210 & 87 \\
\hline${ }^{50} \mathrm{Cr}$ & 110 & 74 & 81 & 51 & 20 & $\ldots$ & 110 & 110 & 130 & 75 & 34 \\
\hline${ }^{52} \mathrm{Cr}$ & 360 & 480 & 390 & 290 & 150 & 27 & 440 & 720 & 560 & 420 & 220 \\
\hline${ }^{53} \mathrm{Cr}$ & 170 & 170 & 140 & 120 & 71 & 17 & 210 & 210 & 190 & 160 & 98 \\
\hline${ }^{55} \mathrm{Mn}$ & 70 & 83 & 76 & 60 & 28 & 47 & 99 & 110 & 100 & 85 & 42 \\
\hline${ }^{54} \mathrm{Fe}$ & 67 & 56 & 60 & 49 & 19 & 46 & 92 & 75 & 87 & 70 & 30 \\
\hline${ }^{56} \mathrm{Fe}$ & 570 & 610 & 580 & 620 & 670 & 680 & 520 & 550 & 520 & 560 & 650 \\
\hline${ }^{57} \mathrm{Fe}$ & 570 & 590 & 620 & 690 & 810 & 100 & 440 & 530 & 540 & 620 & 740 \\
\hline${ }^{59} \mathrm{Co}$ & 140 & 130 & 210 & 190 & 250 & 250 & 170 & 120 & 190 & 180 & 250 \\
\hline${ }^{58} \mathrm{Ni}$ & 280 & 250 & 300 & 350 & 500 & 100 & 170 & 190 & 210 & 230 & 350 \\
\hline${ }^{60} \mathrm{Ni}$ & 730 & 790 & 810 & 920 & 1000 & 950 & 570 & 690 & 690 & 810 & 1000 \\
\hline${ }^{61} \mathrm{Ni}$ & 810 & 860 & 1100 & 1000 & 970 & 930 & 510 & 1000 & 1200 & 1000 & 1000 \\
\hline${ }^{62} \mathrm{Ni}$ & 840 & 750 & 930 & 1100 & 1400 & 160 & 500 & 600 & 670 & 760 & 1300 \\
\hline${ }^{63} \mathrm{Cu}$ & 12 & 18 & 37 & 35 & 20 & 27 & 21 & 19 & 50 & 41 & 24 \\
\hline${ }^{65} \mathrm{Cu}$ & 47 & 67 & 64 & 46 & 32 & 24 & 23 & 99 & 87 & 65 & 39 \\
\hline${ }^{64} \mathrm{Zn}$ & 210 & 300 & 300 & 340 & 190 & 120 & 190 & 420 & 410 & 550 & 240 \\
\hline${ }^{66} \mathrm{Zn}$ & 86 & 88 & 100 & 150 & 160 & 160 & 56 & 79 & 76 & 120 & 160 \\
\hline${ }^{68} \mathrm{Zn}$ & 31 & 35 & 41 & 20 & $\ldots$ & $\ldots$ & 14 & 63 & 59 & 41 & 12 \\
\hline${ }^{69} \mathrm{Ga}$ & 17 & 44 & 19 & 13 & $\ldots$ & $\ldots$ & 12 & 69 & 29 & 21 & $\ldots$ \\
\hline${ }^{70} \mathrm{Ge}$ & 10 & 18 & 21 & 18 & 13 & $\ldots$ & 12 & 44 & 32 & 26 & 16 \\
\hline${ }^{72} \mathrm{Ge}$ & 4.2 & 2.9 & 10 & $\ldots$ & $\ldots$ & $\ldots$ & 2.2 & 11 & 17 & $\ldots$ & $\ldots$ \\
\hline${ }^{74} \mathrm{Se}$ & 21 & 45 & 72 & 35 & 12 & $\ldots$ & 69 & 220 & 130 & 73 & 23 \\
\hline${ }^{78} \mathrm{Kr}$ & 9.0 & 23 & 51 & 42 & 10 & $\ldots$ & 200 & 190 & 88 & 120 & 25 \\
\hline${ }^{80} \mathrm{Kr}$ & 4.2 & 1.6 & 17 & $\ldots$ & $\ldots$ & $\ldots$ & 11 & 26 & 31 & $\ldots$ & $\ldots$ \\
\hline
\end{tabular}


Table 7. Major Production Factors - Double Detonation - Low Mass Dwarfs

\begin{tabular}{|c|c|c|c|c|c|c|c|c|c|c|c|c|c|c|c|}
\hline Species & $9 \mathrm{~A}$ & $9 \mathrm{~B}$ & $9 \mathrm{C}$ & $9 \mathrm{D}$ & $9 \mathrm{E}$ & $8 \mathrm{~A}$ & $8 \mathrm{~B}$ & $8 \mathrm{C}$ & $8 \mathrm{D}$ & $8 \mathrm{E}$ & $7 \mathrm{~A}$ & $7 \mathrm{~B}$ & $7 \mathrm{C}$ & $7 \mathrm{D}$ & $7 \mathrm{E}$ \\
\hline${ }^{28} \mathrm{Si}$ & 210 & 160 & 150 & 140 & 100 & 270 & 260 & 220 & 150 & 46 & 350 & 280 & 300 & 260 & 110 \\
\hline${ }^{32} \mathrm{~S}$ & 350 & 260 & 230 & 210 & 180 & 410 & 440 & 330 & 230 & 94 & 480 & 410 & 420 & 380 & 200 \\
\hline${ }^{34} \mathrm{~S}$ & 47 & 39 & 40 & 40 & 22 & 81 & 40 & 84 & 40 & $\ldots$ & 120 & 81 & 120 & 97 & 21 \\
\hline${ }^{36} \mathrm{Ar}$ & 250 & 190 & 170 & 150 & 130 & 300 & 290 & 220 & 170 & 81 & 370 & 280 & 270 & 250 & 140 \\
\hline${ }^{38} \mathrm{Ar}$ & 25 & 18 & 18 & 16 & 11 & 33 & 26 & 29 & 17 & $\ldots$ & 47 & 33 & 53 & 37 & 11 \\
\hline${ }^{39} \mathrm{~K}$ & 74 & 56 & 32 & 25 & 11 & 140 & 73 & 37 & 50 & $\ldots$ & 290 & 190 & 65 & 46 & 15 \\
\hline${ }^{40} \mathrm{Ca}$ & 480 & 290 & 250 & 220 & 170 & 510 & 400 & 310 & 230 & 110 & 450 & 790 & 380 & 340 & 200 \\
\hline${ }^{43} \mathrm{Ca}$ & 290 & 360 & 270 & 200 & 160 & 290 & 400 & 260 & 230 & 130 & 270 & 940 & 390 & 320 & 220 \\
\hline${ }^{44} \mathrm{Ca}$ & 2000 & 870 & 560 & 370 & 230 & 330 & 1000 & 550 & 390 & 120 & 470 & 3500 & 930 & 670 & 370 \\
\hline${ }^{45} \mathrm{Sc}$ & 18 & 5.3 & 2.6 & 2.2 & 1.8 & 6.2 & 5.5 & 2.8 & 2.4 & 1.4 & 5.1 & 140 & 4.8 & 3.3 & 2.3 \\
\hline${ }^{46} \mathrm{Ti}$ & 370 & 280 & 530 & 580 & 220 & 170 & 310 & 770 & 510 & 120 & 290 & 260 & 970 & 900 & 260 \\
\hline${ }^{47} \mathrm{Ti}$ & 680 & 590 & 800 & 670 & 290 & 180 & 680 & 950 & 690 & 150 & 210 & 600 & 1400 & 1200 & 350 \\
\hline${ }^{48} \mathrm{Ti}$ & 2600 & 2800 & 1700 & 1300 & 750 & 630 & 2700 & 1800 & 1400 & 390 & 1300 & 2500 & 2800 & 2200 & 1100 \\
\hline${ }^{49} \mathrm{Ti}$ & 280 & 170 & 100 & 94 & 83 & 120 & 170 & 130 & 100 & 82 & 130 & 210 & 150 & 140 & 95 \\
\hline${ }^{51} \mathrm{~V}$ & 770 & 920 & 670 & 540 & 280 & 380 & 1100 & 810 & 520 & 150 & 830 & 1200 & 1200 & 950 & 390 \\
\hline${ }^{50} \mathrm{Cr}$ & 200 & 140 & 140 & 130 & 92 & 180 & 210 & 170 & 130 & 50 & 190 & 210 & 210 & 190 & 110 \\
\hline${ }^{52} \mathrm{Cr}$ & 620 & 940 & 800 & 690 & 490 & 680 & 1300 & 930 & 720 & 330 & 740 & 790 & 1200 & 990 & 600 \\
\hline${ }^{53} \mathrm{Cr}$ & 280 & 280 & 210 & 200 & 170 & 270 & 320 & 250 & 210 & 130 & 280 & 280 & 270 & 250 & 180 \\
\hline${ }^{55} \mathrm{Mn}$ & 120 & 140 & 120 & 110 & 89 & 150 & 170 & 140 & 110 & 61 & 150 & 150 & 150 & 150 & 100 \\
\hline${ }^{54} \mathrm{Fe}$ & 120 & 99 & 88 & 81 & 68 & 140 & 150 & 120 & 88 & 44 & 140 & 130 & 120 & 120 & 75 \\
\hline${ }^{56} \mathrm{Fe}$ & 390 & 460 & 480 & 500 & 550 & 290 & 330 & 350 & 470 & 620 & 130 & 240 & 240 & 290 & 510 \\
\hline${ }^{57} \mathrm{Fe}$ & 350 & 430 & 500 & 550 & 650 & 200 & 340 & 450 & 610 & 710 & 68 & 180 & 380 & 490 & 710 \\
\hline${ }^{59} \mathrm{Co}$ & 72 & 130 & 120 & 180 & 170 & 110 & 69 & 170 & 190 & 270 & 8.7 & 120 & 130 & 230 & 170 \\
\hline${ }^{58} \mathrm{Ni}$ & 120 & 140 & 150 & 170 & 190 & 68 & 87 & 100 & 150 & 250 & 21 & 56 & 63 & 85 & 140 \\
\hline${ }^{60} \mathrm{Ni}$ & 400 & 920 & 650 & 730 & 860 & 200 & 410 & 530 & 780 & 1000 & 32 & 180 & 460 & 560 & 950 \\
\hline${ }^{61} \mathrm{Ni}$ & 740 & 920 & 1400 & 1600 & 1300 & 390 & 1100 & 1800 & 1800 & 1100 & 170 & 450 & 2000 & 2300 & 1700 \\
\hline${ }^{62} \mathrm{Ni}$ & 340 & 440 & 520 & 610 & 640 & 180 & 270 & 380 & 540 & 1200 & 38 & 160 & 290 & 380 & 650 \\
\hline${ }^{63} \mathrm{Cu}$ & 16 & 20 & 35 & 63 & 51 & 28 & 30 & 93 & 71 & 35 & 3.2 & 33 & 87 & 140 & 110 \\
\hline${ }^{65} \mathrm{Cu}$ & 66 & 100 & 150 & 140 & 100 & 44 & 150 & 170 & 160 & 55 & 23 & 48 & 230 & 220 & 130 \\
\hline${ }^{64} \mathrm{Zn}$ & 300 & 410 & 690 & 660 & 850 & 170 & 620 & 810 & 980 & 350 & 8.4 & 210 & 1000 & 1100 & 1100 \\
\hline${ }^{66} \mathrm{Zn}$ & 34 & 65 & 73 & 97 & 120 & 30 & 61 & 51 & 80 & 190 & 11 & 26 & 50 & 58 & 190 \\
\hline${ }^{68} \mathrm{Zn}$ & 52 & 51 & 120 & 92 & 68 & 24 & 96 & 130 & 140 & 220 & $\ldots$ & 30 & 190 & 150 & 63 \\
\hline${ }^{69} \mathrm{Ga}$ & 28 & 63 & 81 & 54 & 34 & 27 & 78 & 54 & 56 & 150 & 11 & 23 & 82 & 66 & 43 \\
\hline${ }^{70} \mathrm{Ge}$ & 15 & 35 & 81 & 52 & 40 & 28 & 36 & 58 & 56 & 23 & 16 & 20 & 80 & 75 & 52 \\
\hline${ }^{72} \mathrm{Ge}$ & 8.6 & 7.5 & 32 & 24 & $\ldots$ & 7.6 & $\ldots$ & 35 & 17 & $\ldots$ & 3.2 & 7.2 & 46 & 38 & $\ldots$ \\
\hline${ }^{74} \mathrm{Se}$ & 52 & 130 & 500 & 230 & 130 & 210 & 94 & 210 & 170 & 36 & 100 & 92 & 320 & 290 & 130 \\
\hline${ }^{78} \mathrm{Kr}$ & 23 & 170 & 450 & 200 & 230 & 330 & 26 & 120 & 180 & 37 & 89 & 79 & 160 & 220 & 180 \\
\hline${ }^{80} \mathrm{Kr}$ & 13 & 16 & 92 & 61 & 14 & 30 & $\ldots$ & 45 & 22 & $\ldots$ & 6.1 & 7.4 & 53 & 69 & 13 \\
\hline${ }^{84} \mathrm{Sr}$ & 1.3 & 5.9 & 79 & 14 & $\ldots$ & 9.7 & $\ldots$ & $\ldots$ & $\ldots$ & $\ldots$ & 6.7 & 11 & 1.3 & 11 & $\ldots$ \\
\hline
\end{tabular}


Table 8. Major Production Factors - Helium Shell Detonations

\begin{tabular}{|c|c|c|c|c|c|c|c|c|c|c|c|c|c|c|}
\hline Species & $11 \mathrm{E} 1$ & $11 \mathrm{~F} 1$ & $10 \mathrm{~A} 1$ & 10B1 & $10 \mathrm{E} 1$ & $9 \mathrm{~A} 1$ & $9 \mathrm{C} 1$ & $9 \mathrm{E} 1$ & $8 \mathrm{~A} 1$ & $8 \mathrm{D} 1$ & $8 \mathrm{E} 1$ & 7A1 & $7 \mathrm{~B} 1$ & $7 \mathrm{E} 1$ \\
\hline${ }^{28} \mathrm{Si}$ & $\cdots$ & $\ldots$ & $\ldots$ & $\ldots$ & $\ldots$ & $\ldots$ & $\ldots$ & $\ldots$ & 1.0 & $\ldots$ & 2.6 & 2.7 & 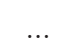 & 1.5 \\
\hline${ }^{32} \mathrm{~S}$ & $\ldots$ & $\ldots$ & 2.4 & $\ldots$ & $\ldots$ & 2.5 & $\ldots$ & $\ldots$ & 6.5 & 1.0 & 1.2 & 17 & 4.2 & 1.0 \\
\hline${ }^{34} \mathrm{~S}$ & 1.3 & $\ldots$ & 1.5 & 2.3 & 2.1 & $\ldots$ & 6.0 & 5.5 & 3.1 & 9.8 & 3.9 & 7.7 & 1.8 & 6.1 \\
\hline${ }^{36} \mathrm{Ar}$ & $\ldots$ & $\ldots$ & 13 & 2.7 & $\ldots$ & 12 & 3.6 & 1.5 & 37 & 2.9 & 1.2 & 83 & 21 & 2.7 \\
\hline${ }^{38} \mathrm{Ar}$ & $\ldots$ & $\ldots$ & $\ldots$ & $\ldots$ & $\ldots$ & $\ldots$ & 1.7 & 1.0 & $\ldots$ & 2.4 & 1.0 & 1.1 & 1.2 & 1.2 \\
\hline${ }^{39} \mathrm{~K}$ & 3.8 & 2.0 & 52 & 18 & 5.6 & 56 & 23 & 5.6 & 130 & 17 & 9.4 & 250 & 170 & 9.0 \\
\hline${ }^{40} \mathrm{Ca}$ & 5.7 & 2.6 & 100 & 51 & 8.9 & 230 & 72 & 30 & 230 & 55 & 17 & 130 & 510 & 46 \\
\hline${ }^{43} \mathrm{Ca}$ & 52 & 23 & 120 & 200 & 78 & 270 & 260 & 140 & 230 & 210 & 130 & 110 & 830 & 220 \\
\hline${ }^{44} \mathrm{Ca}$ & 40 & 17 & 110 & 420 & 61 & 1300 & 550 & 220 & 200 & 370 & 120 & 40 & 3300 & 360 \\
\hline${ }^{45} \mathrm{Sc}$ & $\ldots$ & $\ldots$ & 1.2 & 1.1 & $\ldots$ & 8.8 & 1.2 & $\ldots$ & 2.2 & $\ldots$ & $\ldots$ & $\ldots$ & 130 & $\ldots$ \\
\hline${ }^{46} \mathrm{Ti}$ & 35 & 8.4 & $\ldots$ & 320 & 60 & 4.8 & 590 & 240 & $\ldots$ & 570 & 120 & $\ldots$ & 44 & 250 \\
\hline${ }^{47} \mathrm{Ti}$ & 53 & 21 & 6.0 & 480 & 84 & 97 & 830 & 300 & 9.2 & 710 & 150 & $\ldots$ & 390 & 350 \\
\hline${ }^{48} \mathrm{Ti}$ & 93 & 38 & 4.6 & 1200 & 150 & 1600 & 240 & 630 & 5.6 & 1200 & 300 & $\ldots$ & 1800 & 950 \\
\hline${ }^{49} \mathrm{Ti}$ & 13 & 11 & $\ldots$ & 20 & 18 & 24 & 11 & 19 & $\ldots$ & 25 & 27 & $\ldots$ & 88 & 27 \\
\hline${ }^{51} \mathrm{~V}$ & 36 & 15 & 1.4 & 460 & 59 & 620 & 100 & 230 & 1.3 & 470 & 110 & $\ldots$ & 770 & 340 \\
\hline${ }^{50} \mathrm{Cr}$ & $\ldots$ & $\ldots$ & $\ldots$ & 16 & $\ldots$ & 31 & 2.4 & 7.4 & $\ldots$ & 21 & $\ldots$ & $\ldots$ & 18 & 8.9 \\
\hline${ }^{52} \mathrm{Cr}$ & 26 & 10 & $\ldots$ & 370 & 43 & 420 & 9.5 & 180 & $\ldots$ & 350 & 85 & $\ldots$ & 130 & 270 \\
\hline${ }^{53} \mathrm{Cr}$ & $\ldots$ & $\ldots$ & $\ldots$ & 27 & $\ldots$ & 14 & 2.1 & $\ldots$ & $\ldots$ & 9.5 & $\ldots$ & $\ldots$ & 30 & $\ldots$ \\
\hline${ }^{55} \mathrm{Mn}$ & $\ldots$ & $\ldots$ & $\ldots$ & 26 & $\ldots$ & 33 & $\ldots$ & 14 & $\ldots$ & 26 & 6.0 & $\ldots$ & 9.6 & 20 \\
\hline${ }^{56} \mathrm{Fe}$ & 61 & 99 & $\ldots$ & 10 & 84 & 31 & $\ldots$ & 63 & $\ldots$ & 68 & 140 & $\ldots$ & $\ldots$ & 130 \\
\hline${ }^{57} \mathrm{Fe}$ & 110 & 150 & $\ldots$ & 52 & 160 & 140 & $\ldots$ & 230 & $\ldots$ & 280 & 270 & $\ldots$ & 2.3 & 400 \\
\hline${ }^{59} \mathrm{Co}$ & 75 & 59 & $\ldots$ & 9.6 & 110 & 51 & $\ldots$ & 100 & $\ldots$ & 150 & 180 & $\ldots$ & 2.9 & 110 \\
\hline${ }^{58} \mathrm{Ni}$ & 25 & 53 & $\ldots$ & $\ldots$ & 33 & 11 & $\ldots$ & 22 & $\ldots$ & 30 & 56 & $\ldots$ & $\ldots$ & 26 \\
\hline${ }^{60} \mathrm{Ni}$ & 200 & 220 & $\ldots$ & 73 & 280 & 200 & $\ldots$ & 310 & $\ldots$ & 380 & 480 & $\ldots$ & $\ldots$ & 570 \\
\hline${ }^{61} \mathrm{Ni}$ & 270 & 220 & $\ldots$ & 460 & 400 & 1100 & 3.3 & 940 & $\ldots$ & 1500 & 700 & $\ldots$ & 5.7 & 1400 \\
\hline${ }^{62} \mathrm{Ni}$ & 260 & 290 & $\ldots$ & 36 & 370 & 110 & 1.4 & 110 & $\ldots$ & 180 & 620 & $\ldots$ & 2.2 & 300 \\
\hline${ }^{63} \mathrm{Cu}$ & 18 & 22 & $\ldots$ & 7.1 & 22 & 38 & $\ldots$ & 51 & $\ldots$ & 79 & 34 & $\ldots$ & $\ldots$ & 110 \\
\hline${ }^{65} \mathrm{Cu}$ & 18 & 8.9 & $\ldots$ & 78 & 27 & 150 & 1.4 & 92 & $\ldots$ & 150 & 47 & $\ldots$ & 1.8 & 120 \\
\hline${ }^{64} \mathrm{Zn}$ & 140 & 70 & $\ldots$ & 260 & 190 & 660 & $\ldots$ & 810 & $\ldots$ & 950 & 320 & $\ldots$ & $\ldots$ & 1100 \\
\hline${ }^{66} \mathrm{Zn}$ & 57 & 41 & $\ldots$ & 16 & 81 & 33 & $\ldots$ & 66 & $\ldots$ & 45 & 130 & $\ldots$ & $\ldots$ & 150 \\
\hline${ }^{68} \mathrm{Zn}$ & 7.6 & $\ldots$ & $\ldots$ & 55 & 12 & 120 & $\ldots$ & 70 & $\ldots$ & 140 & 22 & $\ldots$ & $\ldots$ & 64 \\
\hline${ }^{69} \mathrm{Ga}$ & 5.8 & $\ldots$ & $\ldots$ & 59 & 9.3 & 80 & 1.8 & 33 & $\ldots$ & 54 & 15 & $\ldots$ & 1.6 & 43 \\
\hline${ }^{70} \mathrm{Ge}$ & 8.0 & $\ldots$ & $\ldots$ & 36 & 12 & 78 & 1.6 & 37 & $\ldots$ & 51 & 21 & $\ldots$ & 1.6 & 51 \\
\hline${ }^{72} \mathrm{Ge}$ & $\ldots$ & $\ldots$ & $\ldots$ & 10 & $\ldots$ & 33 & $\ldots$ & 6.8 & $\ldots$ & 20 & $\ldots$ & $\ldots$ & $\ldots$ & 6.6 \\
\hline${ }^{75} \mathrm{As}$ & $\ldots$ & $\ldots$ & $\ldots$ & 5.3 & $\ldots$ & 13 & $\ldots$ & $\ldots$ & $\ldots$ & $\ldots$ & $\ldots$ & $\ldots$ & $\ldots$ & $\ldots$ \\
\hline${ }^{74} \mathrm{Se}$ & 12 & $\ldots$ & $\ldots$ & 200 & 23 & 490 & 4.8 & 130 & $\ldots$ & 170 & 35 & $\ldots$ & 3.2 & 130 \\
\hline${ }^{76} \mathrm{Se}$ & $\ldots$ & $\ldots$ & $\ldots$ & 12 & $\ldots$ & 35 & $\ldots$ & $\ldots$ & $\ldots$ & 5.2 & $\ldots$ & $\ldots$ & $\ldots$ & $\ldots$ \\
\hline${ }^{77} \mathrm{Se}$ & $\ldots$ & $\ldots$ & $\ldots$ & 7.9 & $\ldots$ & 26 & $\ldots$ & 6.9 & $\ldots$ & 9.0 & $\ldots$ & $\ldots$ & $\ldots$ & 6.4 \\
\hline${ }^{78} \mathrm{Kr}$ & 10 & $\ldots$ & $\ldots$ & 130725 & 450 & 1.0 & 230 & $\ldots$ & 170 & 37 & $\ldots$ & $\ldots$ & 180 & \\
\hline${ }^{80} \mathrm{Kr}$ & $\ldots$ & $\ldots$ & $\ldots$ & $237 \ldots$ & 92 & $\ldots$ & 14 & $\ldots$ & 22 & $\ldots$ & $\ldots$ & $\ldots$ & 13 & \\
\hline
\end{tabular}


Table 9. Major Production Factors - Double Detonations - Hot WD Models

\begin{tabular}{|c|c|c|c|c|c|c|c|c|}
\hline Species & $11 \mathrm{HD}$ & $10 \mathrm{HC}$ & $10 \mathrm{HCD}$ & $10 \mathrm{HD}$ & $9 \mathrm{HC}$ & $9 \mathrm{HD}$ & $8 \mathrm{HBC}$ & $8 \mathrm{HC}$ \\
\hline${ }^{28} \mathrm{Si}$ & 96 & 160 & 160 & 140 & 210 & 190 & 270 & 250 \\
\hline${ }^{32} \mathrm{~S}$ & 170 & 260 & 240 & 230 & 350 & 290 & 390 & 350 \\
\hline${ }^{34} \mathrm{~S}$ & 18 & 36 & 52 & 45 & 47 & 65 & 75 & 88 \\
\hline${ }^{36} \mathrm{Ar}$ & 130 & 190 & 170 & 160 & 250 & 200 & 270 & 240 \\
\hline${ }^{38} \mathrm{Ar}$ & 8.5 & 16 & 18 & 15 & 25 & 21 & 30 & 27 \\
\hline${ }^{39} \mathrm{~K}$ & 28 & 50 & 61 & 20 & 74 & 32 & 120 & 55 \\
\hline${ }^{40} \mathrm{Ca}$ & 230 & 370 & 250 & 220 & 480 & 280 & 690 & 350 \\
\hline${ }^{43} \mathrm{Ca}$ & 67 & 140 & 250 & 170 & 290 & 250 & 440 & 380 \\
\hline${ }^{44} \mathrm{Ca}$ & 240 & 630 & 710 & 350 & 2000 & 550 & 2500 & 940 \\
\hline${ }^{45} \mathrm{Sc}$ & 4.2 & 7.2 & 4.9 & 1.8 & 19 & 2.6 & 19 & 3.9 \\
\hline${ }^{46} \mathrm{Ti}$ & 8.1 & 14 & 26 & 390 & 370 & 510 & 25 & 470 \\
\hline${ }^{47} \mathrm{Ti}$ & 18 & 41 & 250 & 460 & 680 & 680 & 180 & 830 \\
\hline${ }^{48} \mathrm{Ti}$ & 150 & 220 & 1900 & 1100 & 2600 & 1700 & 840 & 2600 \\
\hline${ }^{49} \mathrm{Ti}$ & 67 & 89 & 120 & 91 & 280 & 120 & 130 & 140 \\
\hline${ }^{51} \mathrm{~V}$ & 66 & 110 & 910 & 500 & 770 & 720 & 440 & 1000 \\
\hline${ }^{50} \mathrm{Cr}$ & 79 & 120 & 110 & 110 & 200 & 140 & 170 & 160 \\
\hline${ }^{52} \mathrm{Cr}$ & 330 & 430 & 1000 & 670 & 620 & 890 & 580 & 1200 \\
\hline${ }^{53} \mathrm{Cr}$ & 170 & 220 & 310 & 200 & 280 & 240 & 270 & 280 \\
\hline${ }^{55} \mathrm{Mn}$ & 75 & 100 & 140 & 110 & 120 & 140 & 130 & 160 \\
\hline${ }^{54} \mathrm{Fe}$ & 67 & 97 & 92 & 86 & 120 & 110 & 130 & 120 \\
\hline${ }^{56} \mathrm{Fe}$ & 580 & 480 & 460 & 490 & 390 & 400 & 280 & 310 \\
\hline${ }^{57} \mathrm{Fe}$ & 510 & 380 & 420 & 500 & 350 & 430 & 170 & 370 \\
\hline${ }^{59} \mathrm{Co}$ & 110 & 72 & 90 & 120 & 72 & 100 & 27 & 70 \\
\hline${ }^{58} \mathrm{Ni}$ & 220 & 150 & 170 & 180 & 120 & 130 & 62 & 81 \\
\hline${ }^{60} \mathrm{Ni}$ & 660 & 480 & 490 & 630 & 400 & 520 & 160 & 430 \\
\hline${ }^{61} \mathrm{Ni}$ & 490 & 340 & 560 & 1100 & 740 & 1300 & 130 & 1400 \\
\hline${ }^{62} \mathrm{Ni}$ & 630 & 420 & 520 & 590 & 340 & 410 & 140 & 270 \\
\hline${ }^{63} \mathrm{Cu}$ & 1.4 & $\ldots$ & 6.7 & 25 & 16 & 34 & 1.8 & 30 \\
\hline${ }^{65} \mathrm{Cu}$ & 10 & 7.4 & 40 & 100 & 66 & 140 & 10 & 180 \\
\hline${ }^{64} \mathrm{Zn}$ & 42 & 31 & 59 & 430 & 300 & 590 & 11 & 740 \\
\hline${ }^{68} \mathrm{Zn}$ & $\ldots$ & $\ldots$ & 8.4 & 89 & 52 & 120 & $\ldots$ & 140 \\
\hline${ }^{69} \mathrm{Ga}$ & $\ldots$ & $\ldots$ & 17 & 46 & 28 & 64 & 8.3 & 91 \\
\hline${ }^{70} \mathrm{Ge}$ & 2.5 & 1.9 & 30 & 45 & 15 & 55 & 17 & 70 \\
\hline${ }^{72} \mathrm{Ge}$ & $\ldots$ & $\ldots$ & 4.6 & 17 & 8.6 & 23 & 2.6 & 21 \\
\hline${ }^{74} \mathrm{Se}$ & $\ldots$ & $\ldots$ & 180 & 200 & 52 & 240 & 160 & 300 \\
\hline${ }^{78} \mathrm{Kr}$ & $\ldots$ & $\ldots$ & 99 & 91 & 23 & 100 & 200 & 150 \\
\hline${ }^{80} \mathrm{Kr}$ & $\ldots$ & $\ldots$ & 10 & 16 & 13 & 21 & 14 & 26 \\
\hline
\end{tabular}


Table 10. Major Production Factors - Single Detonations and Deflagrations

\begin{tabular}{|c|c|c|c|c|c|c|}
\hline Species & 11HD1 & $10 \mathrm{HC} 1$ & 10HCD1 & $8 \mathrm{HBC} 1$ & 8DEFL & 10DEFL \\
\hline${ }^{28} \mathrm{Si}$ & $\ldots$ & $\ldots$ & $\ldots$ & $\ldots$ & $\ldots$ & $\ldots$ \\
\hline${ }^{32} \mathrm{~S}$ & 1.3 & 2.0 & $\ldots$ & 4.0 & 1.4 & 1.0 \\
\hline${ }^{34} \mathrm{~S}$ & $\ldots$ & $\ldots$ & $\ldots$ & 1.3 & $\ldots$ & $\ldots$ \\
\hline${ }^{35} \mathrm{Cl}$ & 4.9 & 8.3 & 9.6 & 19 & 10 & 6.0 \\
\hline${ }^{36} \mathrm{Ar}$ & 7.1 & 11 & 3.5 & 22 & 9.3 & 4.6 \\
\hline${ }^{38} \mathrm{Ar}$ & $\ldots$ & $\ldots$ & $\ldots$ & $\ldots$ & 1.0 & $\ldots$ \\
\hline${ }^{39} \mathrm{~K}$ & 23 & 40 & 70 & 100 & 82 & 47 \\
\hline${ }^{41} \mathrm{~K}$ & 3.5 & 6.2 & 12 & 15 & 28 & 20 \\
\hline${ }^{40} \mathrm{Ca}$ & 89 & 170 & 85 & 420 & 220 & 110 \\
\hline${ }^{42} \mathrm{Ca}$ & $\ldots$ & 1.6 & 29 & 5.1 & 37 & 35 \\
\hline${ }^{43} \mathrm{Ca}$ & 84 & 160 & 320 & 440 & 240 & 160 \\
\hline${ }^{44} \mathrm{Ca}$ & 230 & 610 & 970 & 2400 & 2200 & 1300 \\
\hline${ }^{45} \mathrm{Sc}$ & 2.1 & 4.6 & 7.0 & 15 & 38 & 33 \\
\hline${ }^{46} \mathrm{Ti}$ & 1.4 & 2.9 & 8.4 & 7.0 & 23 & 23 \\
\hline${ }^{47} \mathrm{Ti}$ & 17 & 43 & 270 & 160 & 330 & 280 \\
\hline${ }^{48} \mathrm{Ti}$ & 20 & 66 & 2900 & 430 & 1500 & 2400 \\
\hline${ }^{49} \mathrm{Ti}$ & 1.3 & 3.5 & 170 & 18 & 280 & 490 \\
\hline${ }^{51} \mathrm{~V}$ & 8.6 & 28 & 1200 & 170 & 410 & 540 \\
\hline${ }^{50} \mathrm{Cr}$ & $\ldots$ & $\ldots$ & 35 & 3.9 & 44 & 65 \\
\hline${ }^{52} \mathrm{Cr}$ & $\ldots$ & 2.4 & 410 & 17 & 30 & 100 \\
\hline${ }^{53} \mathrm{Cr}$ & $\ldots$ & $\ldots$ & 140 & 3.5 & 27 & 68 \\
\hline${ }^{55} \mathrm{Mn}$ & $\ldots$ & $\ldots$ & 42 & 1.5 & $\ldots$ & $\ldots$ \\
\hline${ }^{54} \mathrm{Fe}$ & $\ldots$ & $\ldots$ & 2.9 & $\ldots$ & $\ldots$ & $\ldots$ \\
\hline${ }^{56} \mathrm{Fe}$ & $\ldots$ & $\ldots$ & 3.5 & $\ldots$ & $\ldots$ & $\ldots$ \\
\hline${ }^{57} \mathrm{Fe}$ & $\ldots$ & $\ldots$ & 19 & $\ldots$ & $\ldots$ & $\ldots$ \\
\hline${ }^{59} \mathrm{Fe}$ & $\ldots$ & $\ldots$ & 8.4 & 1.2 & $\ldots$ & $\ldots$ \\
\hline${ }^{58} \mathrm{Ni}$ & $\ldots$ & $\ldots$ & 5.1 & $\ldots$ & $\ldots$ & $\ldots$ \\
\hline${ }^{60} \mathrm{Ni}$ & $\ldots$ & $\ldots$ & 9.4 & $\ldots$ & $\ldots$ & $\ldots$ \\
\hline${ }^{61} \mathrm{Ni}$ & $\ldots$ & $\ldots$ & 110 & 5.7 & $\ldots$ & $\ldots$ \\
\hline${ }^{62} \mathrm{Ni}$ & $\ldots$ & $\ldots$ & 26 & 2.7 & $\ldots$ & $\ldots$ \\
\hline${ }^{63} \mathrm{Cu}$ & $\ldots$ & $\ldots$ & 4.6 & $\ldots$ & $\ldots$ & $\ldots$ \\
\hline${ }^{65} \mathrm{Cu}$ & $\ldots$ & $\ldots$ & 17 & 2.8 & $\ldots$ & $\ldots$ \\
\hline${ }^{64} \mathrm{Zn}$ & $\ldots$ & $\ldots$ & 1.7 & $\ldots$ & $\ldots$ & $\ldots$ \\
\hline${ }^{66} \mathrm{Zn}$ & $\ldots$ & $\ldots$ & 8.0 & 1.5 & $\ldots$ & $\ldots$ \\
\hline${ }^{68} \mathrm{Zn}$ & $\ldots$ & $\ldots$ & 1.2 & $\ldots$ & $\ldots$ & $\ldots$ \\
\hline${ }^{69} \mathrm{Ga}$ & $\ldots$ & $\ldots$ & 6.2 & 4.1 & $\ldots$ & $\ldots$ \\
\hline${ }^{70} \mathrm{Ge}$ & $\ldots$ & $\ldots$ & 16 & 4.1 & $\ldots$ & $\ldots$ \\
\hline${ }^{72} \mathrm{Ge}$ & $\ldots$ & $\ldots$ & 4.3 & $\ldots$ & $\ldots$ & $\ldots$ \\
\hline${ }^{74} \mathrm{Se}$ & $\ldots$ & $\ldots$ & 58 & 13 & $\ldots$ & $\ldots$ \\
\hline${ }^{78} \mathrm{Kr}$ & $\ldots$ & $\ldots$ & 37 & 3.6 & 3.6 & 3.6 \\
\hline${ }^{80} \mathrm{Kr}$ & $\cdots$ & $\cdots$ & 4.4 & $\ldots$ & $\ldots$ & $\ldots$ \\
\hline
\end{tabular}


Table 11. Radioactive Progenitors

\begin{tabular}{cccccc}
\hline \hline Species & Made As & Species & Made As & Species & Made As \\
\hline${ }^{43} \mathrm{Ca}$ & ${ }^{43} \mathrm{Sc}{ }^{43} \mathrm{Ti}$ & ${ }^{56} \mathrm{Fe}$ & ${ }^{56} \mathrm{Ni}$ & ${ }^{66} \mathrm{Zn}$ & ${ }^{66} \mathrm{Ge}$ \\
${ }^{44} \mathrm{Ca}$ & ${ }^{44} \mathrm{Ti}$ & ${ }^{57} \mathrm{Fe}$ & ${ }^{57} \mathrm{Ni}$ & ${ }^{68} \mathrm{Zn}$ & ${ }^{68} \mathrm{Se}$ \\
${ }^{47} \mathrm{Ti}$ & ${ }^{47} \mathrm{~V},{ }^{47} \mathrm{Cr}$ & ${ }^{59} \mathrm{Co}$ & ${ }^{59} \mathrm{Cu}$ & ${ }^{69} \mathrm{Ga}$ & ${ }^{69} \mathrm{Se}$ \\
${ }^{48} \mathrm{Ti}$ & ${ }^{48} \mathrm{Cr}$ & ${ }^{60} \mathrm{Ni}$ & ${ }^{60} \mathrm{Zn}$ & ${ }^{70} \mathrm{Ge}$ & ${ }^{70} \mathrm{Se}$ \\
${ }^{49} \mathrm{Ti}$ & ${ }^{49} \mathrm{Cr}$ & ${ }^{61} \mathrm{Ni}$ & ${ }^{61} \mathrm{Zn}$ & $72 \mathrm{Ge}$ & ${ }^{72} \mathrm{Kr}$ \\
${ }^{51} \mathrm{~V}$ & ${ }^{51} \mathrm{Mn}$ & ${ }^{62} \mathrm{Ni}$ & ${ }^{62} \mathrm{Zn}$ & ${ }^{74} \mathrm{Se}$ & ${ }^{74} \mathrm{Kr}$ \\
${ }^{52} \mathrm{Cr}$ & ${ }^{52} \mathrm{Fe}$ & ${ }^{63} \mathrm{Cu}$ & ${ }^{63} \mathrm{Ga}$ & $78 \mathrm{Kr}$ & ${ }^{78} \mathrm{Sr}$ \\
${ }^{53} \mathrm{Cr}$ & ${ }^{53} \mathrm{Fe}$ & ${ }^{65} \mathrm{Cu}$ & ${ }^{65} \mathrm{Ge}$ & $80 \mathrm{Kr}$ & ${ }^{80} \mathrm{Zr}$ \\
${ }^{55} \mathrm{Mn}$ & ${ }^{55} \mathrm{Co}$ & ${ }^{64} \mathrm{Zn}$ & ${ }^{64} \mathrm{Ge}$ & ${ }^{45} \mathrm{Sc}$ & ${ }^{45} \mathrm{Ti}$ \\
\hline
\end{tabular}

